\title{
GAMMA-EMISSION DATA FOR THE CALCULATION OF EXPOSURE RATES FROM NUCLEAR DEBRIS. Volume I. Fission Products
}

by

G. R. Crocker

M. A. Connors
LEGAL NOTICE

Thus report was propared as an account of Government aponasored work Nelther the United A. Meree Commisolion, nor any person acting on behalf of the Commisetion

A. Makes any warranty or representation, expreased or implied, with respect to the eccufacy, completeness, or veefulnesh of the information contasned in this roport, or that the use privetoly omed rights, or

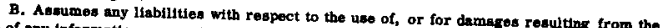

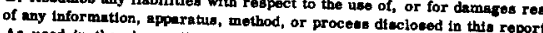

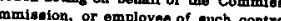

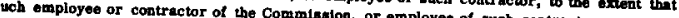
ith the comen or provides accees to, eny information pursuent to his employmeat or contract with the commiston, or ble employment with such contractor
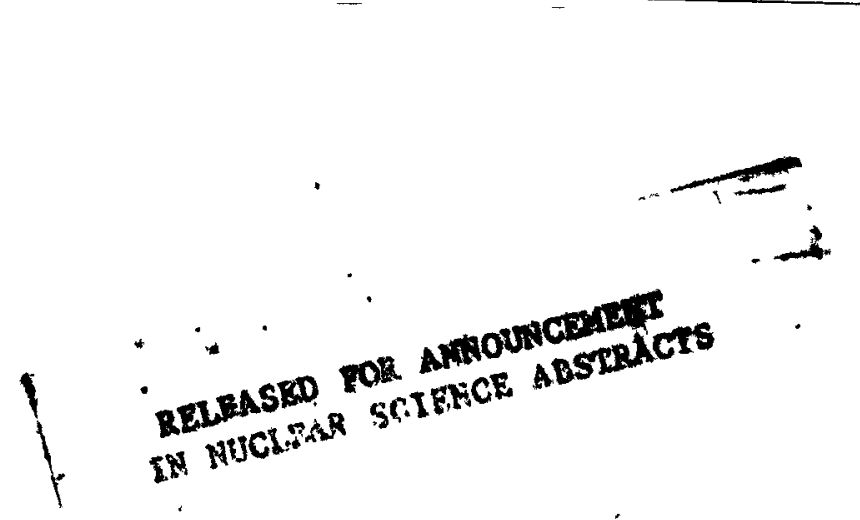

U.S. NAVALARADIOIOGICA L

DE FE N S E LA B O RA T O R Y

SANFRANCISCOBCALIFORNIA・94135 


\section{DISCLAIMER}

This report was prepared as an account of work sponsored by an agency of the United States Government. Neither the United States Government nor any agency Thereof, nor any of their employees, makes any warranty, express or implied, or assumes any legal liability or responsibility for the accuracy, completeness, or usefulness of any information, apparatus, product, or process disclosed, or represents that its use would not infringe privately owned rights. Reference herein to any specific commercial product, process, or service by trade name, trademark, manufacturer, or otherwise does not necessarily constitute or imply its endorsement, recommendation, or favoring by the United States Government or any agency thereof. The views and opinions of authors expressed herein do not necessarily state or reflect those of the United States Government or any agency thereof. 


\section{DISCLAIMER}

Portions of this document may be illegible in electronic image products. Images are produced from the best available original document. 
PHYSICAL CHEMISTRY BRANCH

E. C. Freiling, Head

CHEMICAL TECHNOLOGY DIVISION

R. Cole, Head

\section{ADMINISTRATIVE INFORMATION}

The work reported is part of a project sponsored by the Division of Biology and Medicine of the Atomic Energy Commission under Contract AT (49-7)-1963, Mod. 2.

DDC AVAILABILITY NOTICE

Qualified requesters may obtain copies of this report from DDC.

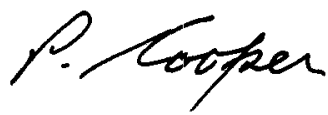




\begin{abstract}
Photon energies and photon abundances have been compiled and summarized for some fission-product and other radionuclides, using data reported in the literature up to June 1963. The data are presented in tabular form, listing photon energies and abundances for gamma rays, beta rays, and $X$ rays emitted. A list of multipliers is also presented for converting activities of the radionuclides to infinite-plane exposure rates.
\end{abstract}




\section{SUMMARY}

The radiation exposure rate from fallout (per disintegration per unit area) depends upon the energies of the photons emitted by the fission-product radionuclides and induced activities and upon the number of photons of each energy emitted in one disintegration. This report is a compilation of the photon energies and abundances for fission products of uranium and plutonium as well as for a few isomers of uranium, neptunium and plutonium which are sometimes present in debris from nuclear weapons. A list of factors for converting radionuclide activities to infinite-plane exposure rate contributions is also presented. 


\section{PREFACE}

This report was originally published as USNRDL Reviews and Lectures ( $R$ \& $L$ ) No. 143. The $R$ \& $L$ series reports are intended primarily for intralaboratory and limited outside distribution or reference. The appearance of this report in the more widely distributed Technical Reports series is in response to numerous outside requests. A second volume, presenting gamma-emission data on some induced radioactivities found in fallout is in preparation. 
CONTENTS

$\underline{\text { Page }}$

ADMINISTRATIVE INFORMATION • . . . . . . . . . . Inside Cover

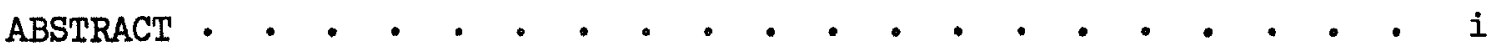
SUMMARY . . . . . . . . . . . . . . . . . . . . ii PREFACE 。 • . . . . . . . . . . . . . . . . . . ili

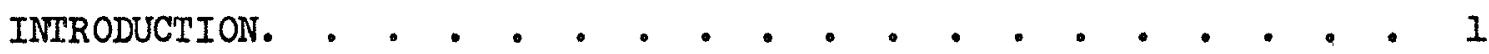

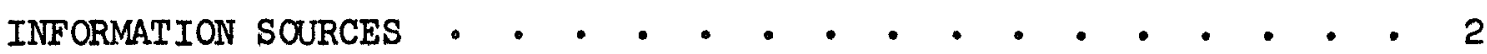
INTERPRETATION OF PUBLISHED DECAY DATA • • . . • • • • • CALCULATION OF THEORETICAL EXPOSURE-DOSE-RATE MULTIPLIERS • • • 6 REFERENCES • • • • • • • • • • • • • • . • • 8 APPENDIX I PHOTON ABUNDANCE SUMMARIES • • . . . . . . . . 9 APPENDIX II DOSE RATE MULTIPLIERS . . . . . . . . . 70 APPENDIX III EXAMPLE OF CALCULATION OF PHOTON ABUNDANCES. . . 74 APPENDIX IV GENERAL FORMULA FOR CONVERTING RELATIVE GAMMA • • ‘78 INTENSITIES TO PHOTON ABUNDANCES

\section{LIST OF FIGURES}

Fig.

1. Decay Scheme for Mass-95 Chain . . . . . . . 75 


\section{INTRODUCTION}

In order to predict exposure-dose rates from calculated fissionproduct activities, it is necessary to know the energies and abundances per disintegration of the gamma photons emitted by the radionuclides. In addition the exposure per unit of photon flux, as a function of energy, must be calculated for the situation of interest. This latter result can be expressed as the dose at a well-defined point (in a standard situation) that results from one disintegration of a specified nuclide. This value can be regarded as a multiplier for converting the activity of that nuclide in a mixture of fission products to the doserate contribution of the nuclide.

C. F. Miller's compliation ${ }^{1}$ has done excellent service as a source of gamma photon energies and abundances for most of the more important fission product gamma emitters. However, in view of the large volume of published data on fission-product decay that has appeared since 1957, it is desirable to up-date and extend Miller's compilation. Many of Miller's gamma photon abundances are merely estimates based on fragmentary data. It is now possible to substitute fairly complete experimental data for some of these and to include others which Miller omitted for lack of information. In other cases, decays have been extensively re-studied and revision of the abundances reported by Miller is desirable.

A review has been made of the most important sources of this kind of information. Since gamma photon abundances are rarely measured directly or reported as such, it was generally necessary to deduce the abundances from reported relative gamma intensities, interpreted in conjunction with energy-level diagrams for the nuclei involved. The resulting collection of current best information on gamma energies, gamma-photon abundances, X-ray photons resulting from gamma conversion, beta energies, and beta particle abundances is presented in Appendix I of this report. The data pertain largely to fission products of uranium, but data also are included for certain isotopes of uranium, neptunium and plutonium that sometimes occur in weapon debris. 
Miller also presented ${ }^{2}$ a list of multipliers for converting activities into dose rates. These have been recalculated using the new gamma abundance data and an improved solution for exposure dose per unit of photon flux. The results are presented in Appendix II.

\section{INFORMATION SOURCES}

Experimental study of beta-decay processes is currently a very active field and the volume of publications on the subject is large. The Nuclear Data Sheets (NDS) published by National Academy of SclencesNational Research Council, including additions and revisions issued to date, formed the basic reference for this compilation. These sheets are prepared by a team of specialists and include a certain amount of critical commentary and evaluation in addition to the data itself.

Many other recently published papers which have not yet been incorporated into NDS were taken into consideration. The principal sources of such papers were Physical Review, Nuclear Physics, Physica, Arkiv for Fysik, Nuovo Cimento, Journal of Inorganic and Nuclear Chemistry, Soviet Journal of Experimental and Theoretical Phys 1cs, and Canadian Journal of Physics. It is believed that these journals account for the major portion of publication in this field.

Much of the material appears in report or thesis form in advance of formal publication in journals. Such sources have not been much utilized for this compliation, partly because they are not easily available and partly because the results presented are of ten tentative in nature.

Nif.ler's estimates have been retained for a number of nuclides for which no new data were encountered. In many other instances, the changes which have been made on the basis of new data are relatively minor. 


\section{INIERPRETATION OF PUBIISHED DECAY DATA}

Data on the gamma and $X$ radiation accompanying beta decay are usually presented in the literature with a view toward establishing the energy level structure of the nuclides involved, rather than toward displaying photon energies and abundances as such. Consequently, It is usually necessary to deduce the photon abundances by interpretation of the decay data in conjunction with a logical decay scheme.

The decay achemes, which are usually provided along with the report of the data, are diagrammatic representations of the processes in which an unstable nuclide is transformed into one of greater stability by emitting a beta particle, with or without accompanying gamma and $X$ radiation. The emission of a beta particle transforms the unstable nuclide into its daughter nuclide. Depending upon the energy of the emitted beta particle, the daughter nuclide may be in its ground. state or in some excited level. In the former case no radiation is emitted, but in the latter case the daughter must de-excite to its ground state by making one or more transitions to lower energy levels. These transitions must be accompanied by the emission of radiation. In general, the radiation consists of one or more gamma photons of the appropriate energies, but an alternative process is the emission of a characteristic $X$ ray, along with an accelerated extranuclear electron, in place of a gamma photon. This process is termed conversion of the gamma photon, and the probability of its occurrence is expressed by a conversion coefficient.

Not all nuclides decay along a single path. Many cases of branching arise, as, for example:

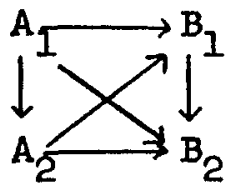

Here $A_{I}$ and $B_{1}$ are metastable isomers of two nuclides whose ground states are $\mathrm{A}_{2}$ and $\mathrm{B}_{2}$. The arrows indicate possible modes of decay, which may or may not be accompanied by emission of gamma rays. $A_{1}$, for instance, decays partly along each of three paths which may involve emission of three different sets of gamma rays. A definite value can be assigned to the percentage of all $A_{1}$ nuclides that 
follows each path. In reporting photon abundances, it is necessary to specify the process for which the figure is applicable. The convention adopted here is: All possible decay paths for a nuclide are considered, and the gamma-photon abundances for each path are multiplied by the appropriate branching fraction and are summed. Further, the paths are considered to extend only to the next stable or metastable state. Thus, in the example, the gamma-photon abundance for $\mathrm{A}_{1}$ Includes alI gamma rays accompanying the transitions $A_{1} \rightarrow A_{2}, A_{1} \rightarrow B_{2}$, and $A_{1} \rightarrow B_{1}$. The radiation accompanying $A_{2} \rightarrow B_{2}$ (as weil as $A_{2} \rightarrow B_{1}$ ) is assigned to $A_{2}$, and that for $B_{1} \rightarrow B_{2}$ is assigned to $B_{1}$.

The data to be interpreted in conjunction with the decay scheme diagram usually consist of beta branching percentages, which indicate the proportion of parent nuclides that decays to the varicus levels in the daughter nuclide, along with relative gamma abundances corresponding to transitions in the daughter nuclide. Occasionally, absolute gamma photon abundances are supplied instead of relative values, and at other times the investigator may choose to report, absolute or relative transition probabilities.

If the data source does not state absolute gamm-photon abundances explicitly, it is necessary to deduce the values by finding a means to normalize reported relative gamma abundances or transition probabilities to absolute photon abundances. If the decay scheme is relatively simple - involving only one or two beta transitions and not many more gamma transitions - the normalization can often be accomplished by inspection.

In more complicated cases, where number of beta transitions and two or more gamm transitions branching from the same level occur, a general formula for absolute gamma abundances may be used, such as the one presented in Append1x IV. However, such formulas are rather cumbersome in application and it frequently happens that some of the parameters are missing and must be estimated. A more direct method, besed on the following princifles, is often useful.

Only one beta particle is emitted per disintegration, and the sum of the branching fractions for the beta transitions must equal one. These transitions can be regarded as populating the various energy levels in the daughter nucleus. The highest lying of the levels can be populated only by beta transitions, but the other levels can also recelve some population by transitions from higher levels. The levels are then depopulated by transitions to lower levels in proportion to the transition probabilities. In the abserce of conversion of gamma photons to $X$-ray photons, the number of transitions are simply proportional to the relative gamma abundances. If conversion occurs, the 
relative gamma abundances must be multiplied by $(1+\alpha)$, where $\alpha$ is the conversion coefficient, in order to obtain relative transition probabilities. Thus, one can start with any level in the daughter nucleus and calculate the number of beta particles and transitions from upper levels which populate it. An equal number of transitions to lower levels must then take place. This number can be properly distributed among gamma photons and $X$-ray photons of various energies, from a knowledge of the conversion coefficients and of either the relative probablilities of transitions to lower levels or the relative gama abundances corresponding to these transitions.

Some of the parameters required by the calculations just described are occasionally missing from the experimental data. When necessary, estimates have been made to arrive at the values presented in this report.

Although the conversion $X$ rays emftted in fission-product decay usually contribute only a very small portion of the total radiation energy, they have been accounted for in this compilation wherever sufficient data were available. X-ray photon emission is not generally reported explicitly in the literature, but conversion coefficients (number of conversion electrons emitted divided by the number of gamma photons emitted) are frequently given. Some of these coefficients are the result of experimental measurements, while others are based on theoretical calculations. Experimental coefficients, if avallable, have been preferred to calculated values for this compilation. In general, conversion has not been assumed to occur unless the $X$ ray has been observed and reported. In a few cases where other strong reasons existed for assuming conversion took place, the theoretical coefficients were used. For most fission products, conversion results in the emission of only $K$-series $X$ rays in significant numbers. The tendency to emit $I_{-\infty}, M-$ and $N$-series $X$ rays increases with atomic number, and in a few cases (notably for the non-firion products $U$, $\mathrm{Np}$, and $\mathrm{Pu}$ ) these have been accounted for.

The $\mathrm{K}$ X-ray energies are taken from Nuclear spectroscopy Tables, 3 Table 7.2-3. These values are derived from experimental measurements and are 10 to $20 \%$ lower than the K-electron binding energies. For the $I-, M-$, and $N$-series $X$ rays such values are not available and the binding energies, taken from Siegbahn, 4 have been used.

For this report, X-ray photon abundances have been calculated as if each conversion electron emitted were accompanied by emission of the corresponding $X$-ray photon. This is not actually the case, since an alternative to $X$-ray emission is the emission of an electron from a higher quantum level. The probability of this process, called Auger 
electron emission, decreases with increasing atomic number and is expressed by a coefficient known as the fluorescent yield of the nuclide. The $\mathrm{K} \mathrm{X}$-ray photon abundances reported here might be improved somewhat by multiplying by the appropriate fluorescent yields, which range from 0.49 to 0.90 for fission products. However, the uncertainty in the conversion data was so large in many cases that the extra refinement in the calculations did not seem warranted. The effect of fluorescence on dose-rate calculations is negligible, in any case. In the case of the I-, M- and N-shells, fluorescent yields are not well known at all, and the corresponding $X$-ray photon abundances reported here should be regarded only as rough numbers.

A detalled example, showing the interpretation of the Nuclear Data Sheet scheme and data for some of the nuclides in the mass-75 decay chain, is presented in Appendix III. It illustrates many of the features of the calculations just described.

The accuracy of the data complled and presented in this report varies widely. The methods used by the original investigators and the equipment available to them were highly diverse. Readers who require estimates of error in the data are referred to the original sources. Many of the reports discuss the accuracy of the measurements in some detail, while others give little information on this point. In view of this situation, no attempt has been made here to assign limits of error to the values reported.

In cases where conflicting data were encountered (and no reason was apparent for preferring one set of results to another), the values reported are those which result in the largest gamma-radiation energy for the nuclide. Since the data are intended for use in predicting hazard from exposure to gamma radiation, overestimation of the gamma abundances seemed preferable to underestimation.

\section{CAICUIATION OF THEORETICAI EXPOSURE-DOSE RATE MULIIPLIERS}

The gama abundances have been assembled primarily for the purpose of computing exposure-dose rates from local fallout. The contribution of each radicactive nuclide to the total dose rate depends on its spectrum of gamma energies and the photon abundances corresponding to each energy. In order to convert photon abundances to roentgens, it is necessary to resort to theoretical calculations of the attenuation of 
gamma rays by matter. As is well known, this attenuation is found to be a function of photon energy. This problem has been extensively studied $5-7$ and the solution is understood in principle. Numerical calculations by machine methods have supplied so-called "build-up" factors 8 wich can be used to arrive at the proper conversion factors. These depend on the particular geometry of the situation of interest. For exposuredose rates from fallout, it is usual to calculate the dose at a point 3 feet above a uniformly contaminated, infinite plane. Conversion factors for this situation, based on the bulld-up factors of cates and Eisenhauer, 8 have been supplied by $S$. Ralney* and used in this report. The factors, calculated for 35 discrete photon energles ranging from $0.1 \mathrm{Mev}$ to $5.0 \mathrm{Mev}$, have been fitted to the following expression:

$$
D=\left(5.97 E-1.21 E^{2}+0.201 E^{3}-0.013 E^{4}\right) \times 10^{-6}
$$

$D$ is the dose rate in roentgens per hour at a point 3 feet above an infinite plane uniformly contaminated with one emitter per square centimeter emitting one photon of energy $E$ per second. This equation is useful for computer work, but for hand calculations the following alternative version is more convenient:

$$
\begin{array}{rlrl}
0<E & <0.2, & D & =5.32 \times 10^{-6} E \\
0.2<E & <3.0, & D & =\left(-0.130+5.77 E-0.643 E^{2}\right) \times 10^{-6} \\
3.0 & <E<5.0 & D & =(3.05+2.78 E) \times 10^{-6}
\end{array}
$$

The calculations on which these equations are based did not cover the range between zero and $0.1 \mathrm{Mev}$. The expressions given above assume a smooth extrapolation of the curve through the origin. A recerit publication by Holme and Stewart 9 suggests that this procedure may be grossly in error for energies below $0.075 \mathrm{Mev}$.

If nuclide $n$ emits $N_{1}$ photons of energy $E_{1}$ per disintegration, $N_{2}$ photons of energy $E_{2}$, etc., then a total dose-rate multiplier, $D_{n}$, for the nuclide can be defined:

$$
D_{n}=D_{1} N_{1}+D_{2} N_{2}+\cdots
$$

If the activity, $A_{n}$, of the nuclide per square centimeter is known, the contribution of the nuclide to the dose rate is $D_{n} A_{n}$ roentgens per hour.

*Of this laboratory, private communication, June 1963. 
The dose-rate multipliers Iisted in Appendix II are presently regarded as tentative, since a re-examination of the method of calculation indicates that it may be subject to improvement, particularly in the region of low energies.

\section{REFERENCES}

1. C. P. Miller, Proposed Decay Schemes for Some Fission-Product and Other Radionuclides. U. S. Naval Radiological Defense laboratory Technical Report USNRDL-TR-160 (27 May 1957).

2. C. F. Milier. Ionization Rate and Photon Pulse Decay of Fission Products From the Slow-Neutron Fission of U235. U. S. Naval Radiological Defense Iaboratory Technical Report USNRDL-TR-247 (4 August 1958).

3. A. H. Wapstra, G. J. N1Jgh, R. van Lieshout. Nuclear Spectroscopy Tables. Interscience Publishers, Inc., New York (1959).

4. K. Slegbahn. Beta- and Gamma-Ray Spectroscopy. Interscience PubI1shers, Inc., New York (1955).

5. I. V. Spencer, U. Fano. Penetration and Diffusion of X-Rays. Calculation of Spatial Distribution by Polynomial Expansion. Phys. Rev. 81:464 (1951).

6. I. V. Spencer, U. Fano. Penetration and Diffusion of X-Rays. Calculation of Spatial Distribution by Polynomial Expansion. $J$. of Res. (NBS) 46:446 (1951).

7. H. Goldstein, J. E. Wilkins, Jr. Calculations of the Penetration of Gamma Rays. Nuclear Development Associates, Inc., Report NYO-3075 (30 June 1954).

8. L. D. Gates, Jr., C. Eisenhauer. Spectral Distribution of Gama Rays Propagated in Air. Armed Forces Special Weapons Project, Technical Analys is Report AFSWP No. 502A (1954).

9. D. A. Holme, K. Stewart. The Gamma Dose-Rate Above an Infinite Plane Source. Atomlc Weapons Research Establishment AWRE Report No. E6/63. May 1963. 


\title{
APPENDIX I
}

\author{
SUMMARY OF PHOTON ENERGIES AND ABUNDANCES
}

Nuclides are arranged by ascending mass number, ordered by atomic number. Metastable-state decays are summarized separately, and these states and their ground states are distinguished by numbers (e.g., As I and As 2, respectively). The half-life of each nuclide is stated below its identification. The source or sources of the data are quoted at the beginning of each summary. The abbreviation NDS is used for the Nuclear Data Sheets compiled and published by National Academy of Sciences-National Research Council. Other literature abbreviations follow standard practice.

The data contained in the columns is indicated by the headings, as follows:

$E(G)$ - Gamma energy in Mev.

$N(G)$ - Corresponding number of photons per disintergration. This column is totalled.

$\mathrm{N}(\mathrm{K})$ - Corresponding number of gammas converted to $\mathrm{X}$-ray photons per disintegration. This column is tatalled.

$N(G) E(G)$ - Product of columns 1 and 2 . This column is totalled.

$E(B)$ - End-point beta-ray energy in Mev.

$N(B)$ - Corresponding number of beta particles per disintegration.

Following the columns the X-ray energy, $\mathrm{E}(\mathrm{K})$, is given. The product of this energy times the total of the $N(K)$ column is given as PRODX. The following totals are then listed:

PH - Total photons emitted per disintegration.

HPH - Total photons emitted per disintegration with energies greater than $0.020 \mathrm{Mev}$.

ED - Total photon energy per disintegration (Mev/dis).

HED - Total photon energy per disintegration (Mev/dis) counting only photons greater than $0.020 \mathrm{Mev}$.

Energies and abundances are printed out only to the third decimal place, although the computer input often carried a fourth place. For this reason, the totals and products (which were machine calculated) are not always completely consistent wth the printout. The discrepancies are not important. 
SOURCE... T.T. THWAITES, PHYS. REV. 129, 1778 (1963),

$722 \mathrm{~N} \quad 30$

$E(B)$

(MEV)

0.246

$N(8)$

(MEV $O$ )

0.001

0.001

0.130

0.014

0.146

PRODX $=0$.

$E D=0.146 M E V / D$

$D H=1.010$

$H P H=1.010$

SOURCE... NOS 59-1-25

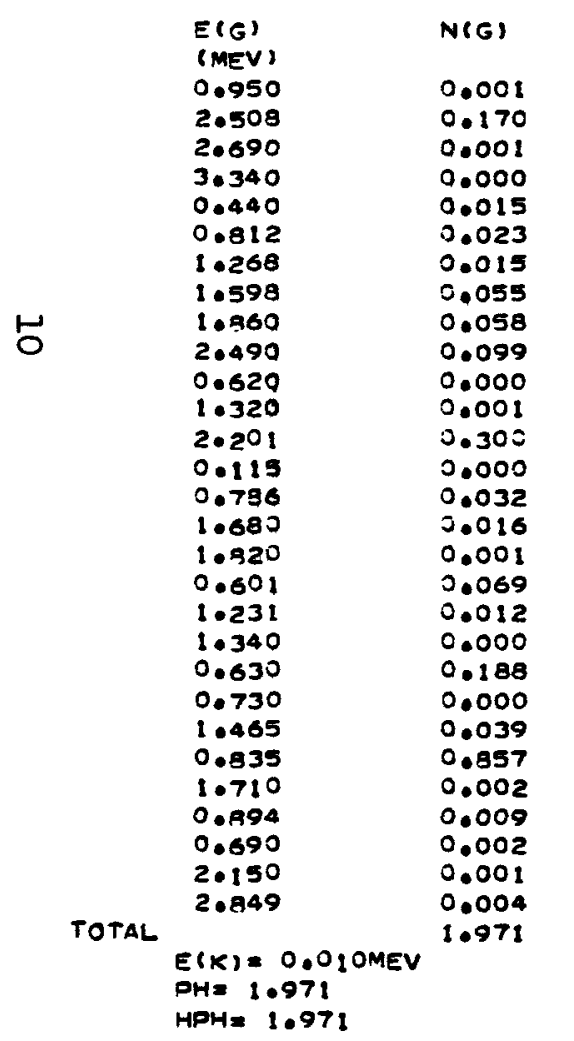

HED = 0.146MEV

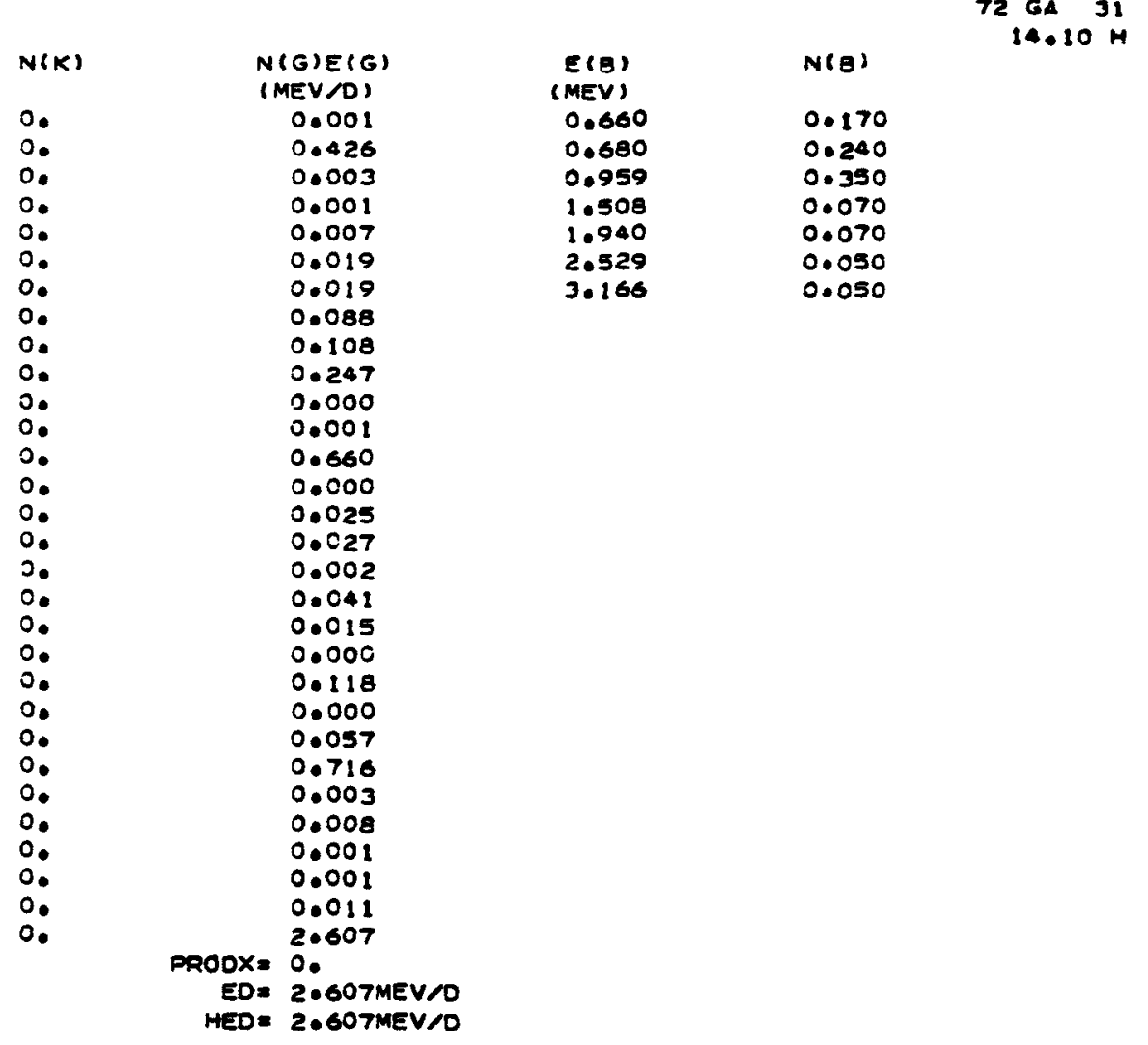


SOURCE... NOS 59-1-37

$\begin{array}{lc}N(K) & N(G) E(G) \\ & \text { (MEV/O) } \\ 0.423 & 0.005 \\ 0.504 & 0.0 \\ 0.927 & 0.005 \\ & \text { ORODX= } 0.009 \\ & E D=0.01 \text { AMEV } \\ & \text { HED }=0.005 M E V / O\end{array}$

73 GEl 32

\begin{tabular}{|c|c|c|}
\hline & $\begin{array}{l}E(G) \\
\text { (MEV) }\end{array}$ & $N(G)$ \\
\hline & $\begin{array}{l}0.054 \\
0.013\end{array}$ & $\begin{array}{l}3.090 \\
3 .\end{array}$ \\
\hline & $\begin{array}{l}F(K)=0.010 M E V \\
O H=1.017 \\
H O H=0.090\end{array}$ & \\
\hline
\end{tabular}

SOURCE... NOS 59-1-37

$\begin{array}{cc}E(G) & N(G) \\ \text { (MEV) } & \\ 0.745 & 0.060 \\ 0.295 & 0.970 \\ 1.040 & 0.010 \\ E(K)=0.010 M E V & 1.040 \\ \text { PHI } 1.040 & \\ \text { HPH }=1.040 & \end{array}$

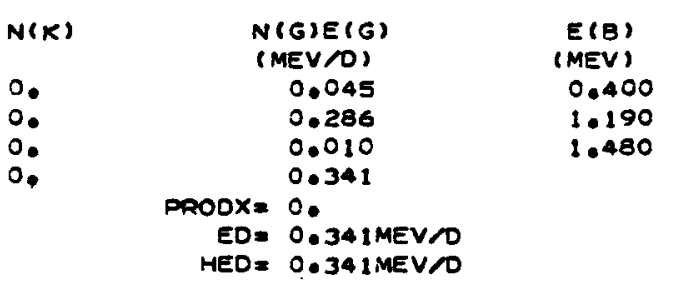

73 GA 31

$N(B)$

0.070

0.910

0.020

HEO $=0.341 \mathrm{MEV} / O$

SOURCE... C.F. VILLER, USNROL-TR-160, (1957).

\begin{tabular}{|c|c|c|}
\hline & $\begin{array}{l}E(G) \\
(M=V) \\
0.700\end{array}$ & $\begin{array}{l}N(G) \\
1.000\end{array}$ \\
\hline JIAL & $\begin{array}{l}E(K)=0.009 M E V \\
P H=1.000 \\
H O H=1.000\end{array}$ & \\
\hline
\end{tabular}

$N(K)$

O.

$+\mathrm{HH}=1.000$
N(GIERG)
(MEV NO)
0.700

0.700

$\begin{aligned} \text { ED } & =0.700 \mathrm{MEV} / \mathrm{O}\end{aligned}$

HED $=0.700$ MEV D

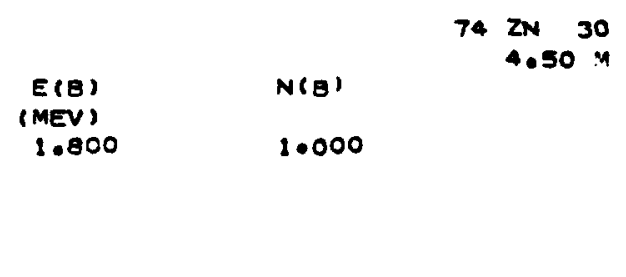


SOURCE... C.YTHIER ET AL.. PHYSICA 251694 (1959).

746431

$\begin{array}{ll}E(G) & N(G) \\ (M E V) & \\ 0.495 & 0.098 \\ 0.600 & 1.401 \\ 0.465 & 0.119 \\ 1.020 & 0.098 \\ 1.100 & 0.077 \\ 1.200 & 0.105 \\ 1.310 & 0.029 \\ 1.450 & 0.094 \\ 1.720 & 0.077 \\ 1.840 & 0.036 \\ 1.960 & 0.094 \\ 2.350 & 0.701 \\ 2.550 & 0.035 \\ 2.720 & 0.049 \\ 2.950 & 0.042 \\ 3.170 & 0.022 \\ 3.330 & 0.024 \\ 3.610 & 0.015 \\ \text { E(K) } & 0.010 M E V \\ O H=3.115 & \\ H P H=3.115 & \end{array}$

5

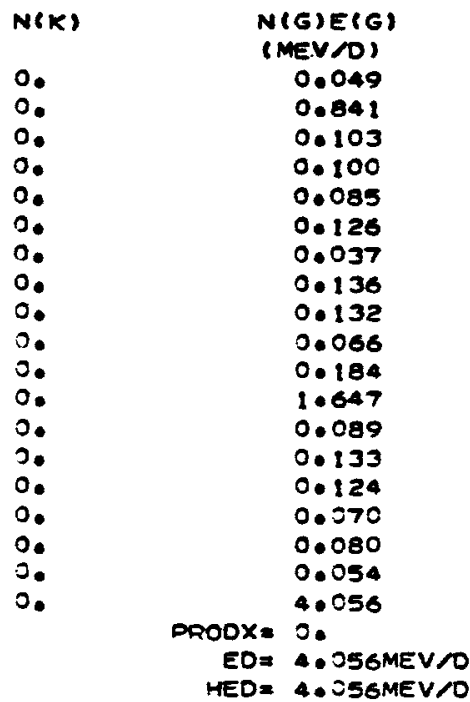

$E(B)$
(MEV)
1.100
1.040
2.050
2.270
2.650
4.300

N(B)

0.110 0.040

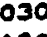

0.100

0.690

0.030

$E D=4.056 \mathrm{MEV} / \mathrm{O}$

75 GEI 32

SOURCE... NOS 59-1-42

N(K)
0.667
0.667

$N(G) E(G)$

(MEV TO)

0.046

$0.139 \quad 0.333$

TOTAL

$E(K)=0.010 M E V$

0.046

$\mathrm{PH}=1.000$

PROOX $=0.007$

$E O=0.053 M E V / O$

$E(B)$

N(B)

$49.00 \mathrm{~S}$

IMEV

75 GE2 32

SOURCE... NOS 59-1-42

$\begin{array}{ll}E(G) & N(G) \\ (M E V) & \\ 0.066 & 0.003 \\ 0.199 & 0.011 \\ 0.264 & 0.110 \\ 0.427 & 0.003 \\ 0.477 & 0.003 \\ 0.628 & 0.001 \\ & 0.134 \\ \text { EN) } & \\ O H=0.138 & \\ H D H=0.134 & \end{array}$

$N(x)$
0.002
0.000
0.002
0.
0.
0.0
0.004

N(G)E(G)
(MEV/D)
0.000
0.003
0.029
0.001
0.001
0.001
0.035

PROOX 0.000

$E D=0.035 M E V / O$

HED $=0.035 M E V / O$

$\begin{array}{ll}\text { E(B) } & N(B) \\ \text { (MEV) } & \\ 0.550 & 0.004 \\ 0.720 & 0.003 \\ 0.920 & 0.114 \\ 0.980 & 0.014 \\ 1.190 & 0.065\end{array}$




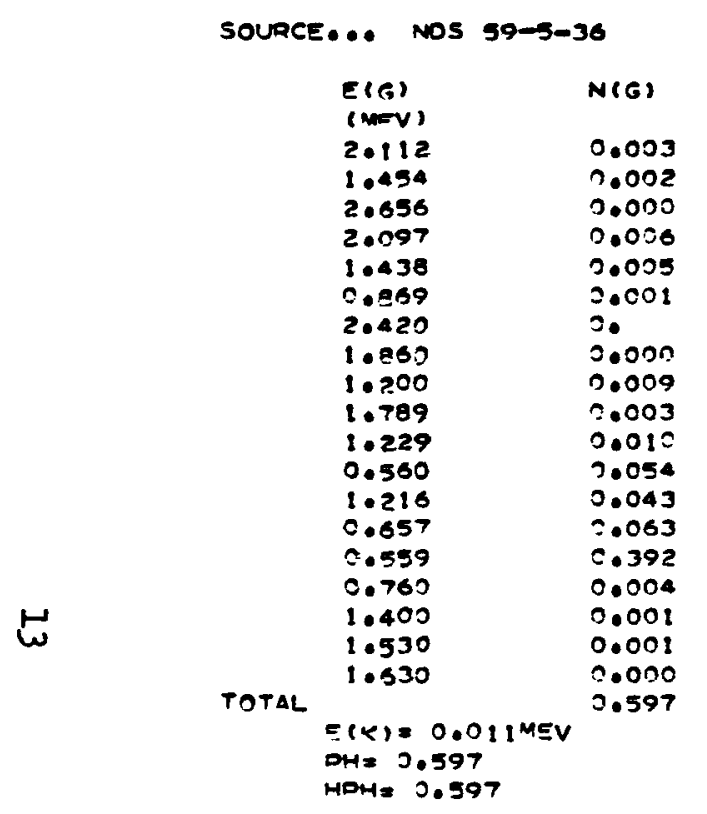

N(K)
0.
$0:$
$0:$
$0:$
$0:$
$0:$
$0:$
$0:$
$0:$
0.
$0:$
$0:$
$0:$
$0:$
0.
$0:$
$0:$
$0:$
$0:$
0.

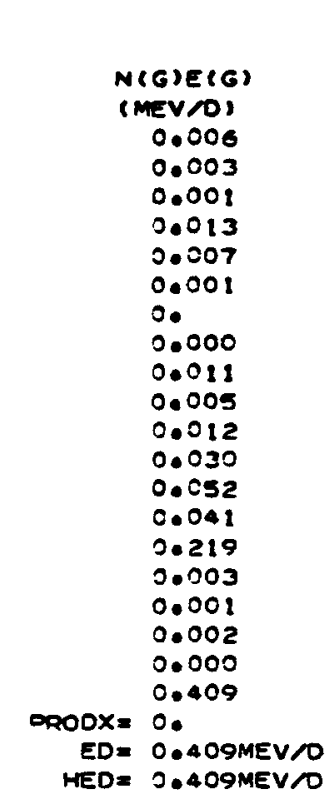

$76 \quad 45 \quad 33$

$26.50 \mathrm{H}$

SOURCE... NOS 59-3-40

$\begin{array}{cc}E(G) & N(G) \\ (M F V) & \\ O .159 & 0.190 \\ 0.215 & 0.180 \\ \text { TOTAL } & 0.370 \\ E(K)=0.011 M E V & \\ O H=0.540 & \\ \text { HPH }=0.370 & \end{array}$

$N(K)$
0.170
0.170
0.170

$E(B)$

(MEV)

0.300

$\begin{array}{ll}0.310 & 0.006 \\ 0.530 & 0.130\end{array}$

$0.550 \quad 0.009$

$1.200 \quad 0.066$

$1.750 \quad 0.036$

3.410

$\begin{array}{ll}2.410 & 0.366 \\ 2.970 & 0.564\end{array}$

HPH $=0.370$ 
SOURCE... NOS 59-3-40

$77 \operatorname{ces} 32$

$11.30 \mathrm{H}$

\begin{tabular}{ll}
$E(G)$ & $N(G)$ \\
$(M E V)$ & \\
2.320 & 0.007 \\
1.960 & 0.024 \\
1.740 & 0.004 \\
0.920 & 0.058 \\
2.020 & 0.024 \\
1.500 & 0.011 \\
1.370 & 0.037 \\
0.500 & 0.108 \\
1.030 & 0.072 \\
1.190 & 0.037 \\
0.709 & 0.108 \\
0.563 & 0.180 \\
0.532 & 0.108 \\
0.416 & 0.252 \\
0.368 & 1.158 \\
0.153 & 0.15 \\
0.210 & 0.355 \\
0.265 & 0.262 \\
0.215 & 0.504 \\
\hline .215 & 2.300
\end{tabular}

TOTAL

$E(x)=0.011$ MEV

H. 2.309

$H P H=2 \cdot 309$

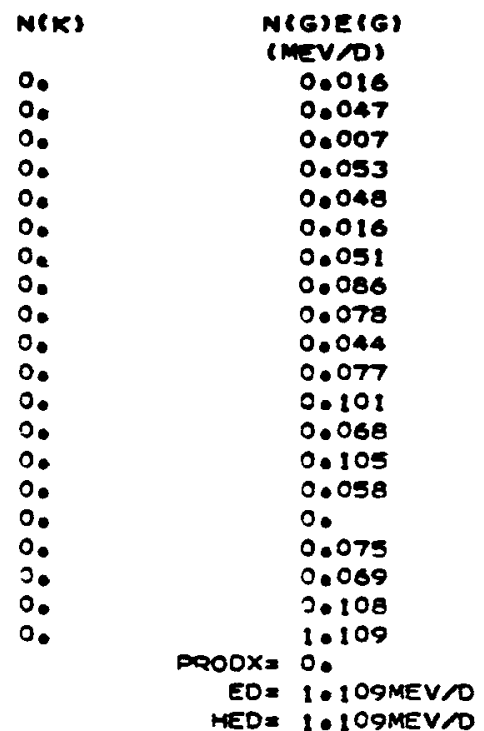

E(B)

$N\left(e^{\prime}\right)$

(MEV)

$0.380 \quad 0.030$

0.150

$1.200 \quad 0.110$

$1.300 \quad 0.040$

$1.560 \quad 0.240$

$2.120 \quad 0.290$

HEO $=1.109 M E V / O$
$2.270 \quad 0.240$

E SOURCE... H. LANGEVIN, J. DUYS. RAD. $16,238129551$. F. RASETTI AN F. C. BOOTH, PHYS. REV. 91. 1192 (1953). NOS 59-3-41 E(G) C.F. MILLER, USNRDL-TR-160, 119571 . (MFV) 0.086

$N(G)$
0.003
0.002
0.023
0.007
0.035
N(K)

N(G)E (G)
(MEV D)
0.000

0.000

0.002

0.245

0.002

0.

TOTAL $0.525 \quad 0.035$

0.004

$$
\mathrm{OH}_{\mathrm{H}}=0.039
$$

HPH= 0.03

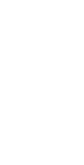

0.000
0.000
0.006
2.004

0.006

0.010

$000 X=0.000$

$E D=0.010 M E V / O$

HED $=0.01$ MMEVTO

SOURCE... C.F. MILLER, USNRDL-TIMIGO, :1957!.

$\begin{array}{cl}E(G) & N(G) \\ (M E V) & \\ 0.450 & 0.990 \\ \text { TOTAL } & 0.990 \\ & (K)=0.011 \text { MEV } \\ \text { PHE } 1.000 & \\ \text { HPH: } 0.990 & \end{array}$

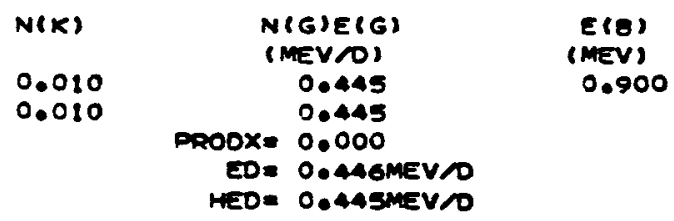

N(E)

1,000
77 AS 33

$38.70 \mathrm{H}$
$N(8)$

0.028

0.001

0.027

0.944

TB Ge 32

$2.10 \mathrm{H}$ 
SOURCE... NOS $59-5-50$

\begin{tabular}{|c|c|c|}
\hline & $\begin{array}{l}E(G) \\
(M E V) \\
0.500\end{array}$ & $\begin{array}{l}N(G) \\
1.000\end{array}$ \\
\hline 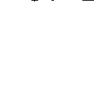 & $\begin{array}{l}E(K)=0.011 \mathrm{MEV} \\
\text { PH=1.000 } \\
\text { HPH=1.000 }\end{array}$ & \\
\hline
\end{tabular}

\begin{tabular}{|c|c|}
\hline$N(K)$ & $\begin{array}{l}\text { N(G)ERG) } \\
\text { (MEV O) }\end{array}$ \\
\hline $\begin{array}{l}0 . \\
0 .\end{array}$ & $\begin{array}{l}0.500 \\
0.500\end{array}$ \\
\hline
\end{tabular}

78 Ast 33

$6.00 \mathrm{M}$

E(B) $\mathrm{N}(\mathrm{B})$

(MEV)

Nis

HED = 0.50OMEV $O$
SOURCE... NOS 59-5-50 (DATA REFERENCED SaVOS ONEY)

$78 \quad 452 \quad 33$

N(S)

0.022

0.021

0.140

0.062

0.097

0.190

$\begin{array}{ll}3.650 & 2.190 \\ 1.270 & 0.472\end{array}$

0.051

0.141

0.031

0.029

0.025
0.229

0.025

0.022

0.037

0.875

PRODX $=0$.

$E D=0.875 M E V / D$

MED $=0.875 M E V / D$

$F H=0.059$

$\mathrm{HPH}=0.959$

SOURCE... NOS 59-2-47

$\begin{array}{cc}E(G) & N(G) \\ \text { (NEV) } & \\ 0.096 & 0.090 \\ \text { TOTAL } & 0.090 \\ \text { ERK) }=0.011 \mathrm{MEV} & \\ \text { PHE } 1.000 & \\ \text { HPH }=0.090 & \end{array}$

SOURCE... NOS 59-1-64

$\begin{array}{ll}E(G) & N(G) \\ (M E V) & \\ 0.103 & 0.104 \\ \text { TOTAL } & 0.104 \\ E(K)=0.011 M E V & \\ \text { OHE } 1.000 & \\ \text { HPH }=0.104 & \end{array}$

$\begin{array}{cc}N(K) & N(G) E(G) \\ & \text { (MEVNO) } \\ 0.910 & 0.009 \\ 0.910 & 0.009 \\ & \text { MOOX } \\ & 0.010 \\ & E D=0.019 M E V N O \\ \text { MED }=0.009 M E V / O\end{array}$

$E(B)$

(MEV)

N(B)

79 SEI 34

$3.89 \mathrm{M}$

81 SeI 34

$57.00 \mathrm{~m}$

\begin{tabular}{|c|c|c|}
\hline$N(K)$ & & $\begin{array}{l}N(G) E(G) \\
(M E \vee O)\end{array}$ \\
\hline $\begin{array}{l}0.896 \\
0.896\end{array}$ & & $\begin{array}{l}0.011 \\
0.011\end{array}$ \\
\hline
\end{tabular}


SOURCE... NOS 59-1-67

$\begin{array}{cl}E(S) & N(G) \\ (N F V) & \\ 0.554 & 0.727 \\ 0.519 & 0.454 \\ 0.599 & 0.309 \\ 0.777 & 0.909 \\ 0.827 & 0.273 \\ 1.044 & 0.318 \\ 1.317 & 0.309 \\ 1.475 & 0.182 \\ \text { TOTAL } & 3.481 \\ \text { E(K) }=0.013 \text { NEV } & \\ \text { PHE } 3.481 & \\ \text { HPH }=3.481 & \end{array}$

$N(x)$
0.
0.
0.
0.
0.
0.
0.
0.

N(G)E(G)
(MEV/DI
0.403
0.281
0.216
0.706
0.226
0.332
0.407
2.268
2.839
PROOX $=0.0$
ED= $2.839 M E V / O$
HED $=2.839 M E V / O$

E(B)

N(g)

J.444

10000

SOUDCE... NOS 59-1-77

$\begin{aligned} E D & =2.839 \mathrm{MEV} / O \\ M E D & =2.839 \mathrm{MEV} / \mathrm{O}\end{aligned}$

83 SE 134

$69.00 \mathrm{~s}$

$\begin{array}{ll}E(G) & V(G) \\ \text { (MEV) } & \\ 2.022 & 0.028 \\ 1.010 & 0.071 \\ 0.350 & 0.014 \\ 0.350 & 0.009\end{array}$

TOTAL

$E(K)=0.012 \mathrm{MEV}$

2.123

DW 0.123

$404=0.123$

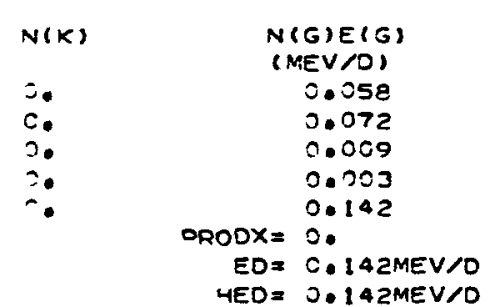

$E(B)$

(MEV)

1.500
3.400

$N(B)$

0.100

0.900

$E D=C .142 M E V / D$

SOURCE... R.G. COCHRAN AN 'ע.W. PRATT, PHYS. REV. 113, BS2 (19S9).

83 SE2 34 $25.00 \mathrm{M}$

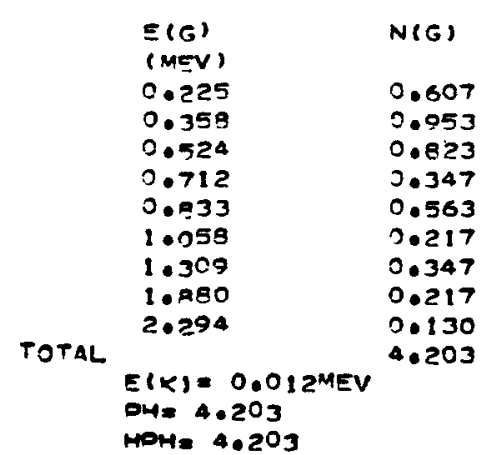

N(K)
0.
$0:$
$0:$
$0:$
$0:$
$0:$
$0:$
$0:$

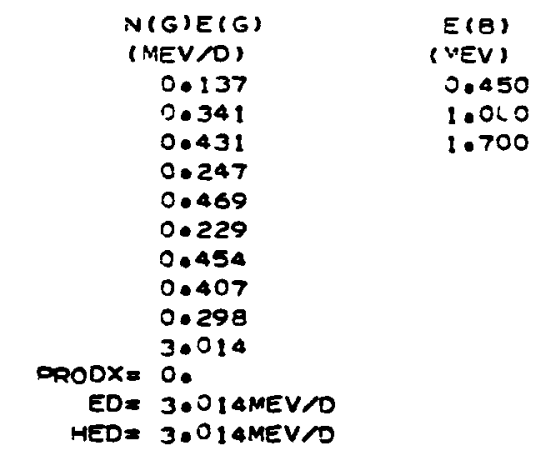

$N(B)$

0.130

0.830

0.040

$H E D=3.014 M E V / D$ 
SOURCE... NOS 59-1-77

$\begin{array}{cl}E(G) & N(G) \\ (M E V) & \\ 0.046 & 0.160 \\ 0.032 & 1.000 \\ 0.009 & 1.000 \\ \text { TOTAL } & 2.160 \\ E(K)=0.013 M E V & \\ \text { OHI } 2.200 & \\ \text { HOW }=1.160 & \end{array}$

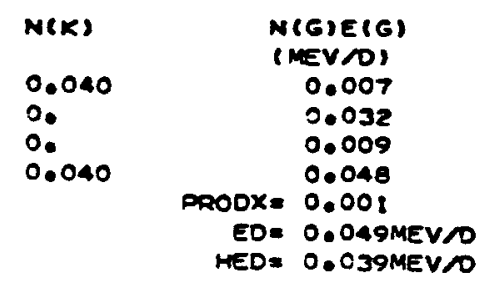

SOURCE... NOS 59-1-77

$\begin{array}{cl}E(G) & N(G) \\ (P P V) & \\ 0.032 & 1.000 \\ 0.009 & 1.000 \\ \text { TOTAL } & 2.000 \\ E(K)=0.013 \text { MEV } & \\ \text { PHF } 2.000 & \\ \text { HPH }=1.000 & \end{array}$

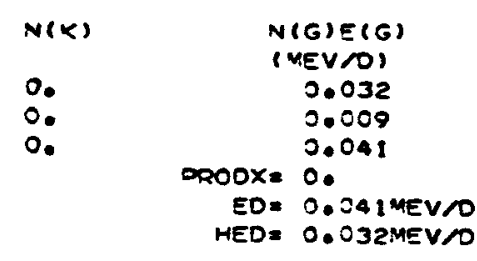

SOURCE... NDS 5-2-5 (DEC. 1962)

83 BR 35

$2.40 \mathrm{H}$

$E(B)$

n(e)

0.200
0.800

0.910

$\begin{aligned} \text { EDD } & =0.049 \mathrm{MEV} / \mathrm{O} \\ \text { HED } & =0.039 \mathrm{MEV} / \mathrm{O}\end{aligned}$

$\begin{array}{cc}N(G) E(G) & E(B) \\ \text { (MEVNO) } & \text { (MEV) } \\ 0.079 & 0.500 \\ 0.410 & 0.830 \\ 0.091 & 0.980 \\ 0.325 & 1.390 \\ 0.001 & 1.810 \\ 0.015 & 2.800 \\ 0.039 & 3.830 \\ 0.053 & 4.680 \\ 0.194 & \end{array}$

N(s)

Q4 BR 35

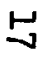

$93 \times R 136$

N(B)
0.030

0.190 0.020 0.140 0.015 0.150 0.140

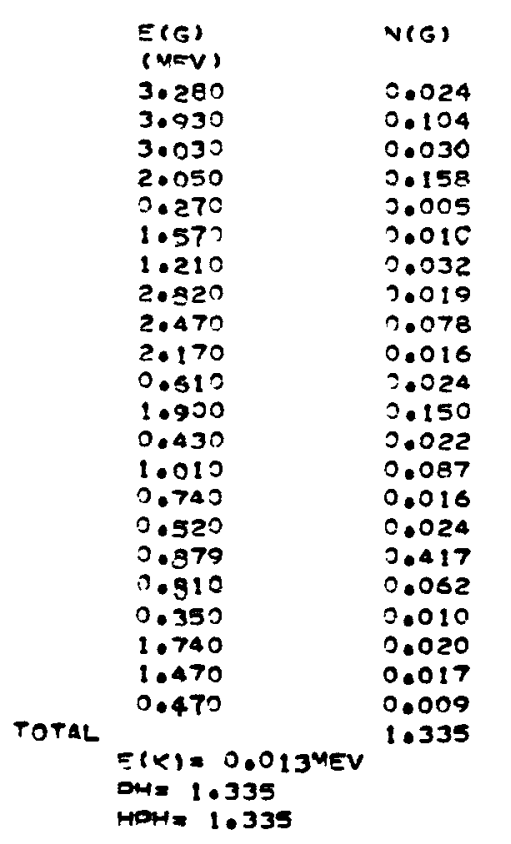
0.194

0.014

0.285

0.285

0.009

0.012

0.012

0.366

0.051

0.004

0.035

0.025

$2 \cdot 148$

Droox $=0$.

ED $=2.1$ ABMEV $/ O$
HED $=2.1$ ABMEV $/ O$ 


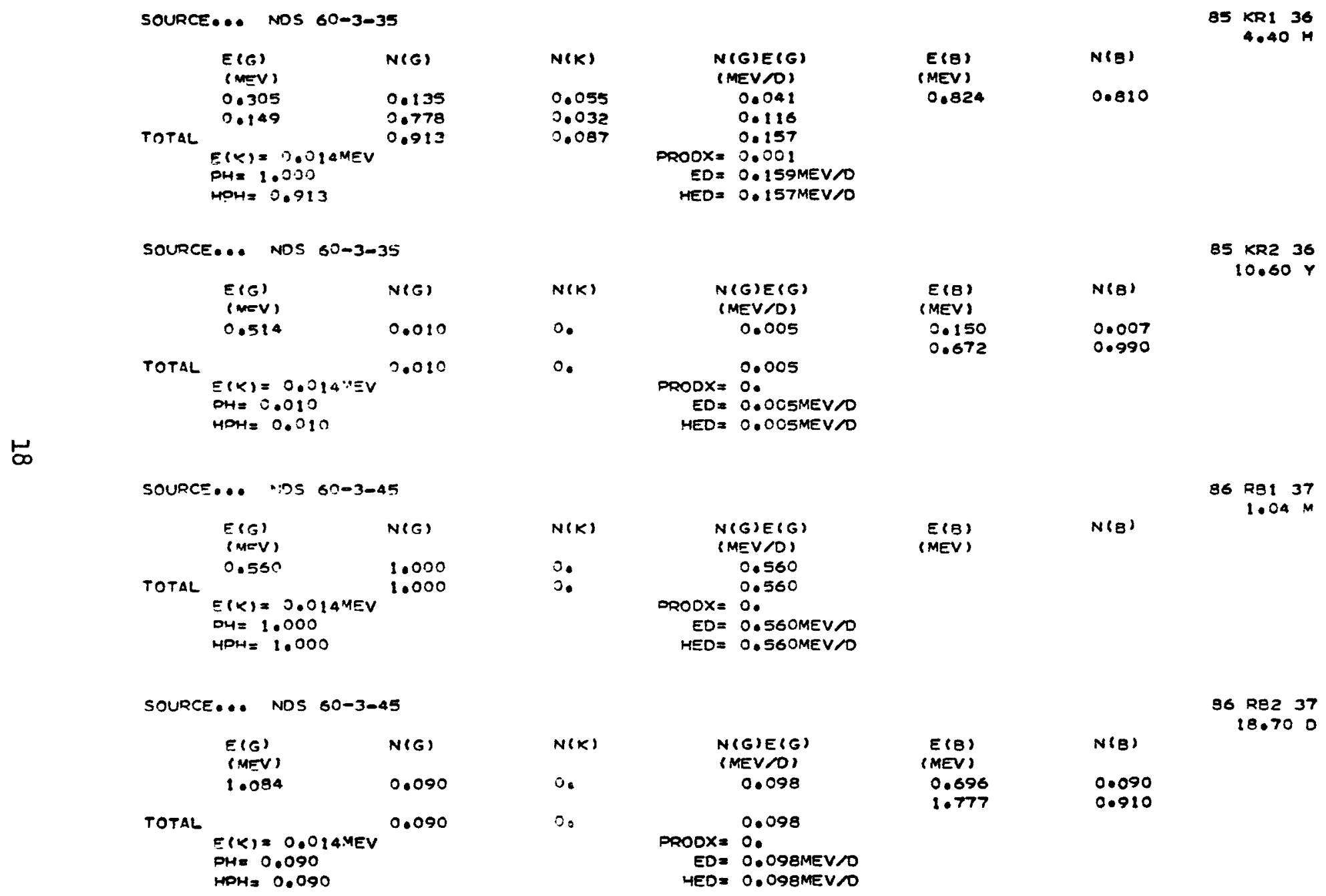


SOURCE... NDS $60-3-56$

\begin{tabular}{|c|c|c|}
\hline & $\begin{array}{l}E(G) \\
\text { (MFV) }\end{array}$ & $N(G)$ \\
\hline & $\begin{array}{l}0.403 \\
0.047\end{array}$ & $\begin{array}{l}0.524 \\
0.100\end{array}$ \\
\hline & $\begin{array}{l}2.050 \\
2.570\end{array}$ & $\begin{array}{l}0.031 \\
0.220\end{array}$ \\
\hline TOTAL & $\begin{array}{l}E(K)=0.014 \mathrm{MEV} \\
3 \mathrm{~W}=9.975 \\
\text { HOM= }=0.875\end{array}$ & 0.375 \\
\hline
\end{tabular}

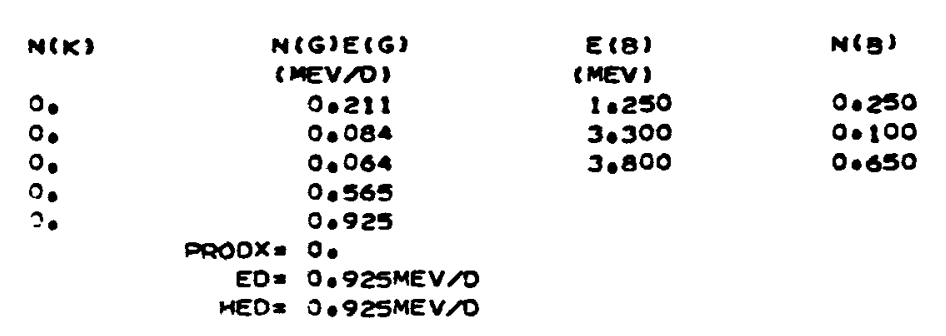

$87 \mathrm{KR} 36$

$78.00 \mathrm{~m}$

SOUPCE... WS 63-3-67

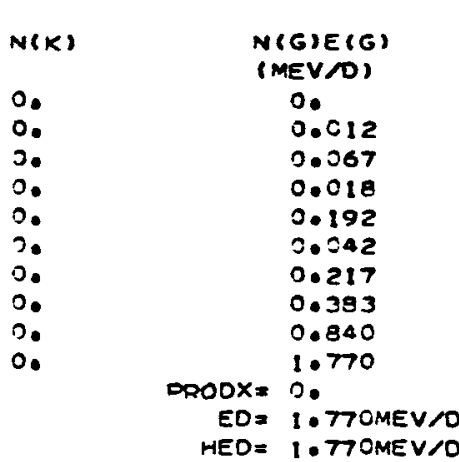

$\begin{array}{ll}0.129 & 0.070 \\ 0.165 & 0.070\end{array}$

$0.191 \quad 0.350$

$0.363 \quad 0.049$

1.19 ? 0.035

1.55 ? 0.140

?.19n 0.175

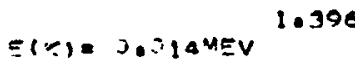

$D_{4}=1.306$

HOH $=1.396$

SOURCE... NDS B.T-3-67

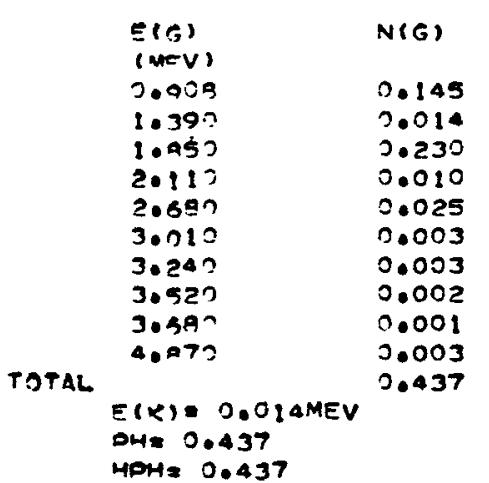

HED $=1.77 O M E V / O$

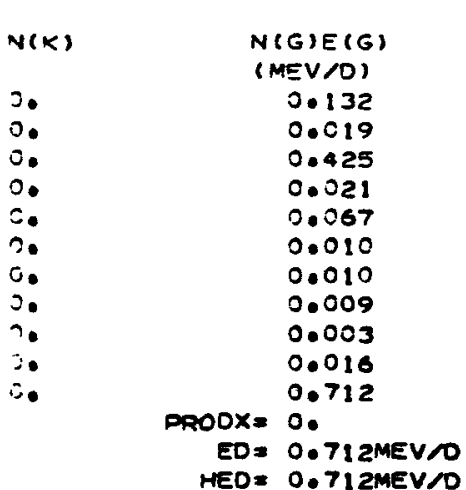

$E(B)$

(MEV)

$0.520 \quad 0.700$

0.100

0.200

88 KR 36

$2.80 \mathrm{H}$

88 RB 37 $N(8)$

(MEV)

2.500

$3.400 \quad 0.240$

$5.200 \quad 0.760$

-0 .

0.060 


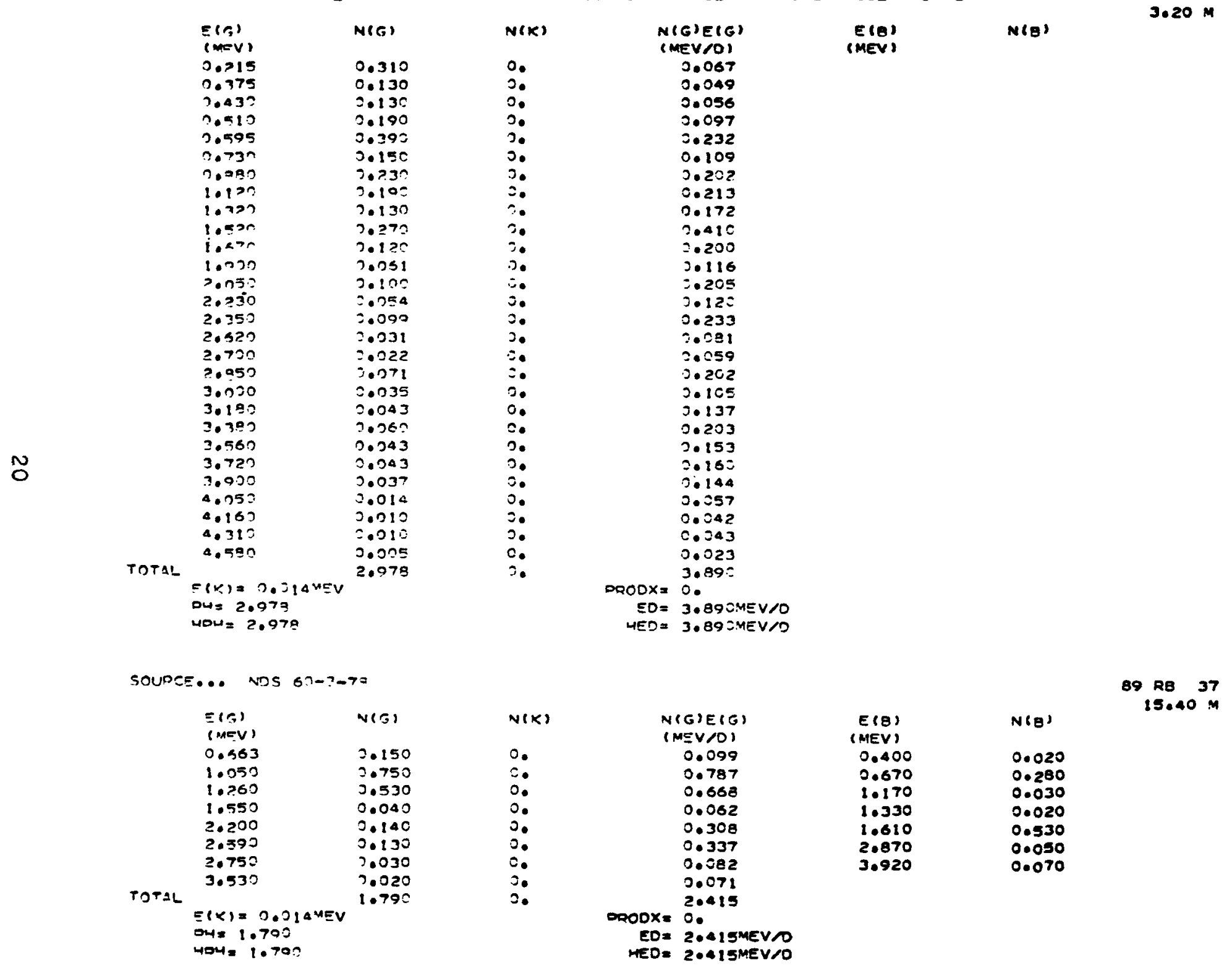


SOURCE... M.A. WAHLGREN AND W.W MEINKE, J. INORG. NUCL. CHEM, 24,1527 (1962).

$90 \underset{33.005}{36}$

$N(\theta)$

E(B)
(MEV)

(MEV OO)

0.084

0.017

0.362

0.737

0.431

0.214

1.844

PRODX $=0$.

$E D=1.844 M E V / D$

$\begin{aligned} E D & =1.844 M E V / D \\ H E D & =1.844 M E V / O\end{aligned}$

$04=2.450$

HPH $=2.480$

SOURCE... NDS $60-5-7 C$

(MEV)

0.150

$\begin{array}{ll}0.748 & 0.270 \\ 0.930 & 0.030\end{array}$

$\begin{array}{ll}0.748 & 0.270 \\ 0.930 & 0.030\end{array}$

$\begin{array}{ll}1.025 & 0.300 \\ 1.413 & 0.150\end{array}$

TOTAL

$E(K)=0.015 \mathrm{MEV}$

0.900 HPH $=0.900$

SOURCE... NDS $60-5-70$

\begin{tabular}{|c|c|c|}
\hline & $\begin{array}{l}E(G) \\
\text { (MEV) }\end{array}$ & $N(G)$ \\
\hline DTAL & 0.551 & $\begin{array}{l}0.956 \\
0.956\end{array}$ \\
\hline & $\begin{array}{l}E(K)=0.015 \mathrm{MEV} \\
D H=1.000 \\
H P H=0.956\end{array}$ & \\
\hline
\end{tabular}

SOURCE... NDS $60-5-70$

$\begin{array}{cc}E(G) & N(G) \\ (M E V) & \\ l .208 & 0.003 \\ \text { TOTAL } & \\ E(X)=0.016 M E V & 0.003 \\ \text { PHE } 0.003 & \\ \text { HPH }=0.003 & \end{array}$

$N(K)$

o.

0.

$N(K)$
0.
$0:$
$0:$
0.
0.

$N(K)$

0.044

0.044

$$
\text { PROOX }=\begin{array}{r}
0.527 \\
0.527
\end{array}
$$

$E D=0.527 \mathrm{MEV} / \mathrm{O}$

MED $=0.527 M E V / D$

$O O X=0$

$\begin{aligned} E D & =0.846 \mathrm{MEV} / \mathrm{D} \\ \text { HED } & =0.846 \mathrm{MEV} / \mathrm{D}\end{aligned}$

N(GIE(G)
(MEV/D)
0.004
0.004
PROOX $=0.0$
ED $=0.004 M E V N D$
HED $=0.004 M E V N D$

$E(B)$

(MEV)

1.090

1.090

2.030

2.030

91 SR 38

$N(B)$

0.070

0.330

0.290

0.040

0.270

$91 \times 139$
$50.00 \mathrm{M}$

$E(B)$

$N(B)$

$91 \quad 4239$

E(B)

$N(B)$

0.003

0.997 
SOURCE... NDS 60-5-9O

$\begin{array}{ll}E(G) & N(G) \\ (M E V) & \\ O .230 & 0.035 \\ 0.440 & 0.041 \\ 1.370 & 0.900 \\ \text { TOTAL } & 0.976 \\ E(K)=0.015 \text { MEV } & \\ \text { OH }=0.976 & \\ \text { HOH }=0.976 & \end{array}$

$\begin{array}{lc}N(K) & N(G) E(G) \\ & (M E V / O) \\ 0 . & 0.008 \\ 0 . & 0.018 \\ 0 . & 1.233 \\ 0 . & 1.259 \\ & \text { DOOX= } 0.1 \\ & E D=1.259 M E V / O \\ & \text { HED }=1.259 M E V / O\end{array}$

SOURCE... M.E. QUNKER ET AL., DHYS. REV. 127. 844 (1962).

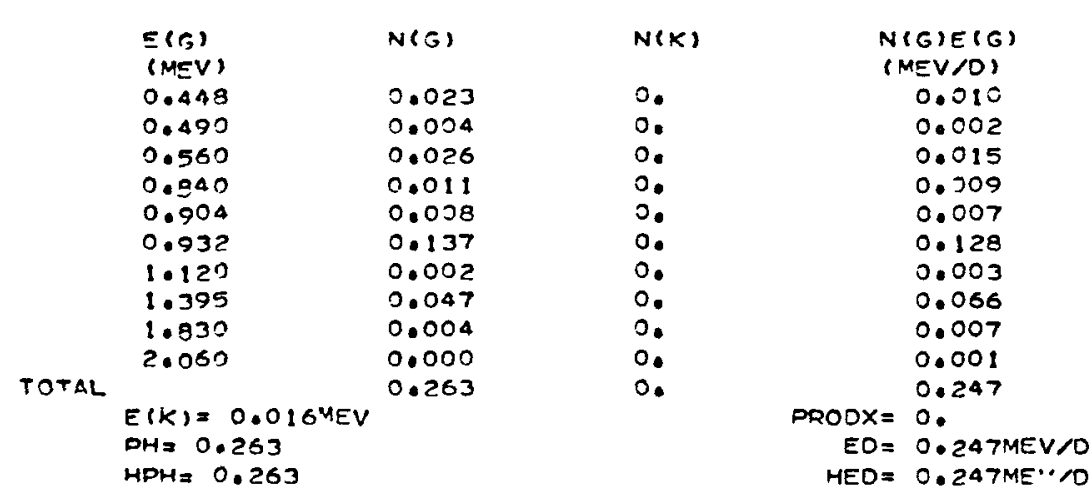

SOURCE... C.F. MILLER, USNRDL-TR-160, (1957).

92 SR 38 $2.70 \mathrm{H}$

$E(B) \quad N(B)$

$0.545 \quad 0.900$

$1.500 \quad 0.100$

$E D=1.259 M E V / D$

HED $=1.259 M E V / O$
$92 Y 39$

N(B)

0.015

0.023

0.031

0.062

0.003

0.008

$3.53 \mathrm{H}$

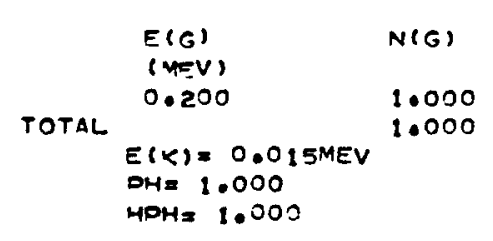

$\mathrm{MH}=1.000$
$N(K)$

O.

$N(G) E(G)$
$(M E V / 0)$
0.200
0.200
PRODX= 0.
ED $=0.200 M E V / O$
MED $=0.200 M E V / O$

HED $=0$. 2OOMEV/O
$E(B)$
(MEV)
2.150

2.150
2.260

2.710

1.320

1.510

$\begin{aligned} E D & =0.247 M E V / D \\ H E D & =0.247 M E \cdot / D\end{aligned}$ 
SOURCE... NOS 60-5-91

$93 Y 39$

$E(G) \quad N(G)$ (MEV) 2.430
2.180 $2 \cdot 180$

2.180
1.950

1.950

1.420

0.490

0.035

0.665

0.367

1.200

TOTAL

2.05

$E(k)=0.016 \mathrm{MEV}$

$04=0.130$

$\mathrm{HOH}=0.129$

SOUPCE... NDS $60-5-92$

w

$\begin{array}{cl}E(G) & \text { NSG) } \\ \text { (MEV) } & \\ 0.029 & 1.000 \\ \text { TOTAL } & 1.000 \\ E(K)=0.017 \text { MEV } & \\ \text { FH= } 1.000 & \\ \text { HDH }=1.000 & \end{array}$

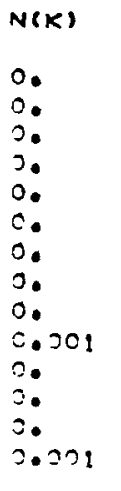

N(G)E(G)

(MEV/O)

0.001

0.002

0.034

0.010

0.003

0.000

0.022

0.005

0.017

0.000

0.005

0.001

$E D=0.103$ HEV $/ D$

$H E D=0.103$ MEV $/ 0$

\section{$E(B)$ \\ (MEV) \\ 0.450 \\ 0.710 \\ 1.470
1.950 \\ 2.620 \\ 2.890}

N(B)

0.001

0.018

0.009

0.030

0.039

0.900

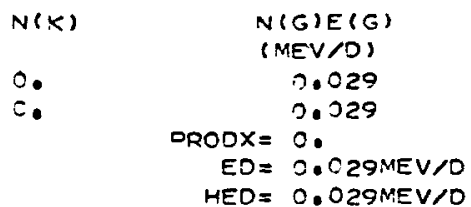

$E(B)$

(MEV)

93 NB2 41 $3.70 \mathrm{Y}$

$N(B)$

SOURCE... J.D. KNIGHT, O.C. HOFFMAN, B.J. DROPESKY AND D.E. FRASCO,

$94 Y^{3} 39$

$N(B)$

(MEV)

TOTAL

$\begin{array}{ll}E(G) & N(G) \\ \text { (MEV) } & \\ 0.560 & 0.060 \\ 0.920 & 0.430 \\ 1.130 & 0.050 \\ 1.650 & 0.024 \\ 1.900 & 0.016 \\ 2.130 & 0.024 \\ 2.570 & 0.015 \\ 2.940 & 0.007 \\ 3.060 & 0.013 \\ 3.930 & 0.011 \\ E(K)=0.016 \text { MEV } & 0.650 \\ \text { DH= } 0.650 & \\ M P H=0.650 & \end{array}$

NeK
0.
$0:$
$0:$
$0:$
$0:$
0.
$0:$
$0:$

0.034

0.396

0.056

0.040

0.030

0.051

0.039

0.020

0.040

0.039

0.744

PROOX $=0$.

$E D=0.744 M E V N O$
HED $=0.744 M E V / O$ 
SOURCE... NOS 60-s-119

952040

$\begin{array}{cl}E(E) & \text { N(E) } \\ (M E V) & 0.550 \\ 0.726 & 0.430 \\ 0.760 & 0.020 \\ 0.235 & 1.000 \\ \text { TOTAL } & \\ & \end{array}$

\begin{tabular}{|c|c|c|}
\hline $\begin{array}{l}\text { N(K) } \\
0.000 \\
0.001 \\
0.0\end{array}$ & $\mathbf{N}$ & $\begin{array}{c}\text { N(G)E(G) } \\
\text { (neVNo) } \\
0.399 \\
0.327 \\
0.005\end{array}$ \\
\hline 0.001 & $\begin{array}{r}\text { PAODX }= \\
\text { ED= } \\
\text { HED }=\end{array}$ & $\begin{array}{l}0.731 \\
=0.000 \\
=0.731 \mathrm{~m} \\
=0.731 \mathrm{~m}\end{array}$ \\
\hline
\end{tabular}

SOURCE... WS $60-5-120$

$\begin{array}{ll}E(3) & N(G) \\ (M=V) & \\ 0.235 & 1.000 \\ \text { TOTAL } & 1.050 \\ E(K)=0.017 M E V & \\ \text { OUE } 1.000 & \\ \text { HOH }=1.000 & \end{array}$

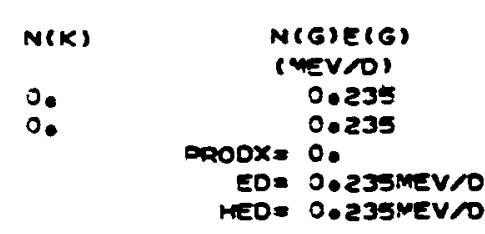

SOUPCE... NOS 60-3-120

\begin{tabular}{|c|c|c|}
\hline & $\begin{array}{l}E(G) \\
\text { (MEV) } \\
\text { SETGQ }\end{array}$ & $N(G)$ \\
\hline TOTAL & & 3.990 \\
\hline & $\begin{array}{l}E(K)=0.01 \text { I MEV } \\
\text { PH= } 0.990 \\
\text { MOH }=0.990\end{array}$ & \\
\hline
\end{tabular}

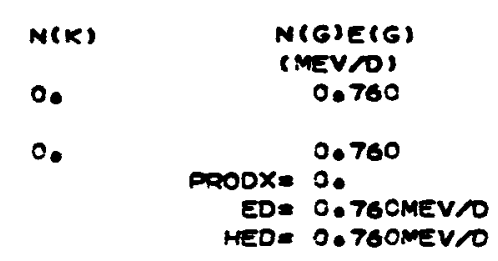

SOURCE... NDS $60-3-33$

$\begin{array}{cl}\text { E(e) } & \text { N(E) } \\ \text { (MEV) } & \\ 0.360 & 0.430 \\ 0.396 & 0.530 \\ 0.005 & 0.020 \\ 1.130 & 0.004\end{array}$

45.000

CDE 0.73IMEV/O

HED $=0.035 \%$

E(Q)

(MEV)

95 NBI 41

$90.00 \mathrm{H}$

95 Ne2 4 !

$35.00 \mathrm{D}$

\section{$E(B)$}

(MEV)

0.160
0.930

$N(8)$

0.990

0.010

$\begin{aligned} & E D=0.76 C M E V / O \\ & \text { HED }=0.760 \mathrm{MEV} / O\end{aligned}$

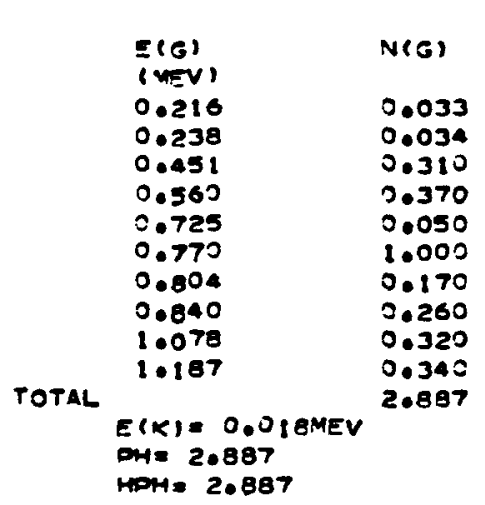

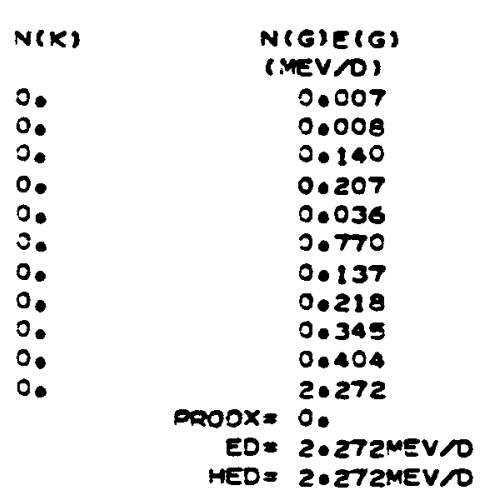

$E(\theta)$

(MEV)

0.370

N(B)

96 Ne 41

0.700

0.080

0.920

$E D=2.272 M E V N O$
HED $=2.272 M E V / O$ 
SOURCE... NOS 60-6-43

$\begin{array}{ll}E(G) & N(G) \\ \text { IMEV } & \\ 1.020 & 0.010 \\ 1.150 & 0.020 \\ 1.720 & 0.005 \\ 1.770 & 0.005 \\ \text { TOTAL } & 0.040 \\ \text { E(K) } & 0.017 M E V \\ \text { DHE } 0.040 & \\ \text { MPH }=0.040 & \end{array}$

SOUTCE... NDS 6O-6-43

$\begin{array}{cc} & \\ N(K) & N(G) E(G) \\ 0 . & \text { (MEV/D) } \\ 0.010 \\ 0 . & 0.023 \\ 0 . & 0.009 \\ 0 . & 0.009 \\ 0 . & 0.051\end{array}$

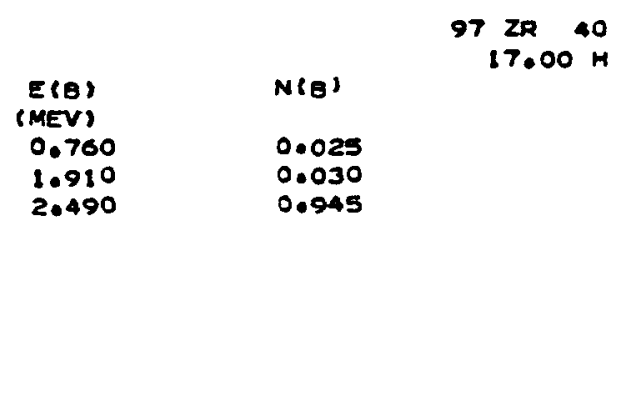

RODX $=0$.

$E D=0.051 M E V / D$

HED $=0.051 \mathrm{MEV} / \mathrm{O}$
E(G) N(G)

(MEV) $\quad 0.986$
TOTAL
$E(K)=0.017 M E V$
H= 1.000
0.986
HOH $=0.986$

N

SOURCE... NOS 60-6-43

$\begin{array}{cl}E(G) & N(G) \\ (M E V) & \\ 1.020 & 0.010 \\ 0.665 & 0.990 \\ \text { TOTAL } & 1.000 \\ E(K)=0.01 \text { MEEV } & \\ \text { PHE } 1.000 & \\ \text { MPHE }=1.000 & \end{array}$

SOURCE... NOS 61-1-49

$\begin{array}{cl}E(G) & N(G) \\ \text { (MEV) } & \\ 0.950 & 0 . \\ 0.740 & 0.100 \\ 0.780 & 0.040 \\ 0.372 & 0.010 \\ 0.181 & 0.037 \\ 0.140 & 0.068 \\ \text { TOTAL } & 0.255 \\ \text { E(K) }=0.019 M E V & \\ \text { PHE } 0.264 & \\ \text { MPH }=0.255 & \end{array}$

97 NBI 41

$1.00 \mathrm{~m}$

$E(\theta)$

N(B)

(MEV $O$ )

$$
0.739
$$

PROOX $=0.000$

ED $=0.74$ CMEV $/ O$

HED $=0.739 \mathrm{MEV} / \mathrm{O}$

(MEV)

N

97 N82 41

73.004

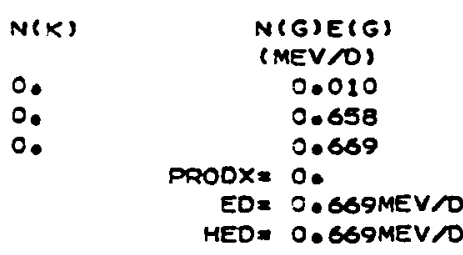

E(B)

(MEV)

0.930

1.267

$N(8)$

0.010

0.990

$\begin{aligned} E D & =0.669 M E V / O \\ \text { HED } & =0.669 \mathrm{MEV} / 0\end{aligned}$

$N(K)$
0.
0.
0.0
0.0003
0.0003
0.000
0.009
$N(G) E(G)$
(MEV/D)
0.
0.074
0.031
0.004
0.007
0.010
0.125

PRODX $=0.000$

$E D=0.125 M E V / D$

HED $=0.125 M E V / D$

$E(\theta)$
$(M E V)$
0.450
0.870
1.230

99 MO 42

$N(\theta)$

0.140

0.010

0.850 
SOURCE... NOS $6:-1=49$

$99 \mathrm{TCl} 43$ $6.00 \mathrm{H}$
(MEV) 0.004

$0.002 \quad 0.986$

TOTAL

$0.140 \quad 0.900$

$E(K)=0.019 \mathrm{MEV}$

OM= 1.976

004

SOURCE... NOS $61-1-63$

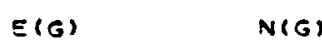

(MFV)

0.538

$\begin{array}{ll}0.530 & 1.000 \\ 0.600 & 1.000\end{array}$

TOTAL

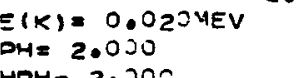

$E(K)=0.0204 E V$

$O H=2.000$

SOURCE... NDS $61-2-26$

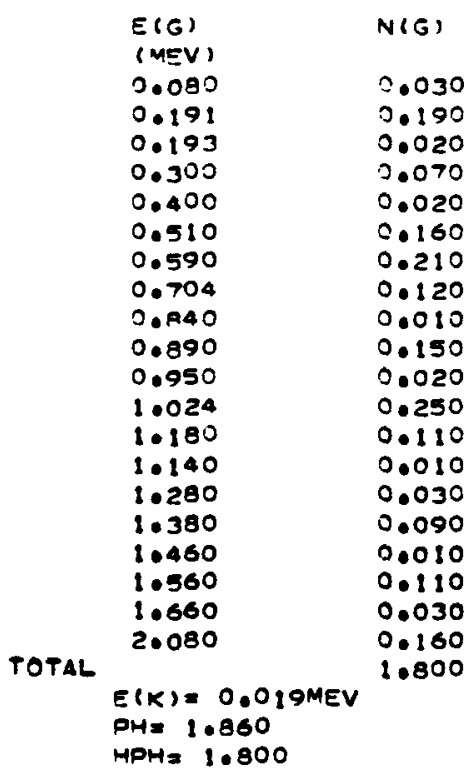

$E(B)$

$N(G) E(G)$

MEV DI

0.
0.086
0.086
0.086

0.086

0.002

0.126

$000 x=0.129$

$E D=0.130 M E V / O$

$H E D=0.127 M E V R$

$N(G) E(G)$
(MEVNO)
0.538
0.600

$1 \cdot 138$

DRODX $=0$ :

$E D=1.138 \mathrm{MEV} / \mathrm{O}$

HEO $=1 \cdot 138 M E V / O$

$E(\theta)$

(MEV)

2.200

2.900

3.370

$N(\theta)$
100 TC 43

$16.00 \mathrm{~s}$

$N(B)$

$-\infty$
$-\infty$

1.000

MPH $=1.800$

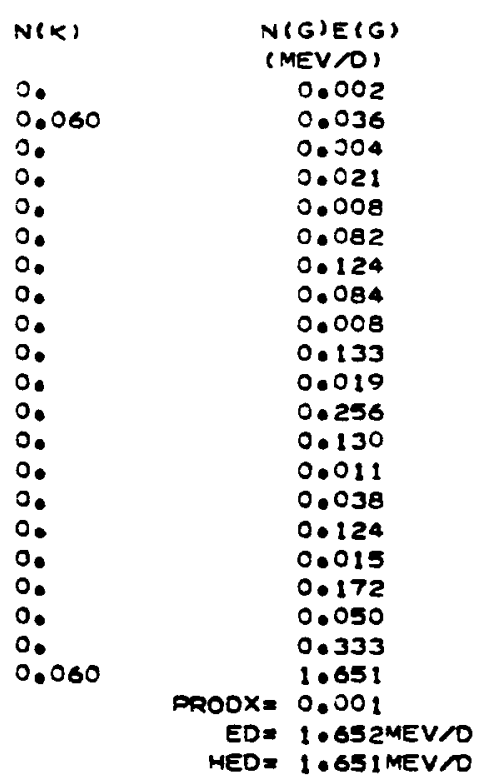

$101 \mathrm{MO} 42$

$\begin{array}{ll}E(B) & N(B) \\ (M E V) & \\ 0.600 & 0.030 \\ 0.700 & 0.380 \\ 0.800 & 0.130 \\ 1.200 & 0.110 \\ 1.600 & 0.250 \\ 2.230 & 0.100\end{array}$


SOURCE... NOS $61-2-26$

101 Te 43

$\begin{array}{ll}E(G) & N(G) \\ (M E V) & \\ 0.939 & 0.002 \\ 0.385 & 0.016 \\ 0 . A 46 & 0.003 \\ 0.720 & 0.011 \\ 0.635 & 0.009 \\ 0.545 & 0.076 \\ 0.235 & 0.007 \\ 0.410 & 0.004 \\ 0.307 & 0.910 \\ \text { TOTAL } & 1.038 \\ E(K)=0.020 M E V & \\ \text { PHE } 1.039 & \\ H P H=1.038 & \end{array}$

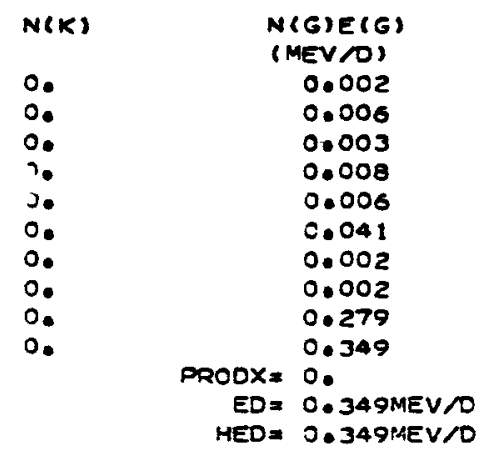

SOURCE... C.F. MILLER, USNROL-TR-160, (1957).

$\begin{array}{cc}E(G) & N(G) \\ (M E V) & \\ 1.100 & 1.000 \\ \text { TOTAL } & 1.000 \\ E(K)=0.020 \text { MEV } & \\ \text { PHE } 1.000 & \\ H P H=1.000 & \end{array}$

N(K)

$\begin{aligned} E D & =0.349 M E V / D \\ M E D & =0.34914 E V / O\end{aligned}$

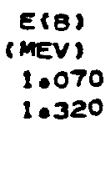

$\begin{array}{ll}\text { S(G) } & N(G) \\ (\text { MEV }) & \\ 0.610 & 0.065 \\ 0.555 & 0.005 \\ 0.498 & 0.880 \\ 0.440 & 0.005 \\ 0.053 & 0.007 \\ \text { TOTAL } & 0.952 \\ E(K)=0.021 \mathrm{MEV} & \\ P H=0.975 & \\ \text { HPH }=0.975 & \end{array}$

$N(K)$
0.
0.005
0.005
0.008
0.013

SOURCE... NOS 61-3-62

$N(G) E(G)$
IMEV/DI
1.100
1.100
RODX $=0$.
ED $=1.100$ MEV $/ 0$
HED $=1.100$ MEV $/ 0$

102 TCl 43

$N(B)$

10000
103 RU 44 $39.70 \quad 0$

$\begin{array}{ll}\text { E(G) } & N(G) \\ \text { (MEV) } & \text { N(G) } \\ 0.040 & 0.024 \\ \text { TOTAL } & 0.024 \\ \text { E(K) }=0.021 \text { MEV } & \\ \text { PH }=1.000 & \\ \text { MOM } & =1.000\end{array}$

$\begin{array}{cc}N(K) & N(G) E(G) \\ & \text { (MEV/D) } \\ 0.976 & 0.001 \\ 0.976 & 0.001 \\ & \text { PRODX } 0.020 \\ & E D=0.021 \text { MEV } O \\ & \text { HED }=0.021 \text { MEV/D }\end{array}$


SNURCE... NOS 61-4-4

104 RHI 45

$4.40 \mathrm{M}$

$\begin{array}{ll}E(G) & N(G) \\ \text { (MEV) } & \\ 0.051 & 0.370 \\ 0.077 & 0.020 \\ 0.096 & 0.020 \\ \text { TOTAL } & 0.410 \\ E(K)=0.022 M E V & \\ \text { OH= } 1.097 & \\ \text { MOH }=1.097 & \end{array}$

$\begin{array}{lc}N(K) & N(G) E(G) \\ & (N E V N O) \\ 0.329 & 0.019 \\ 0.358 & 0.002 \\ 0.0 & 0.002 \\ 0.687 & 0.022\end{array}$

$E(B)$

(MEV)

N(B)

0.001

DRODX $=0.09$

$E D=0.037 M E V / O$

HED $=0.037 M E V / C$

SOURCE... NOS $61-4-4$

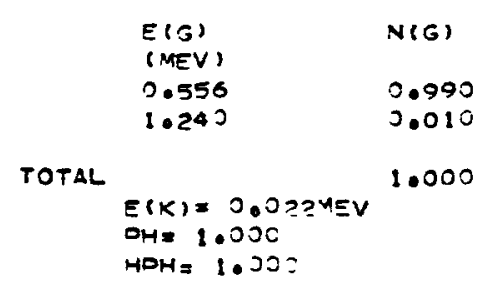

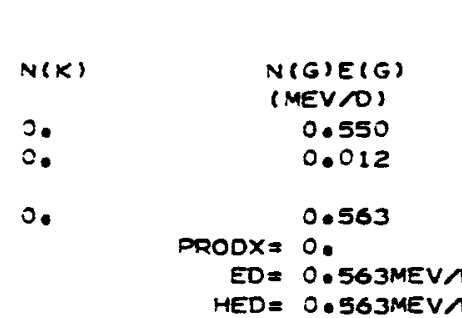

104 RH2 45 $42.00 \mathrm{~S}$

$\begin{array}{ll}E(B) & N(B) \\ \text { (MEV) } & \\ 0.640 & 0.001 \\ 1.880 & 0.018 \\ 2.440 & 0.980\end{array}$

$E D=0.563 M E V / O$

HED $=0.563 M E V P$

$\begin{array}{ll}E(G) & N(G) \\ \text { (MEV) } & \\ 0.130 & 0.011 \\ 2.210 & 0.050 \\ 0.260 & 0.065 \\ 0.317 & 0.130 \\ 0.400 & 0.070 \\ 0.475 & 0.210 \\ 0.670 & 0.175 \\ 0.725 & 0.500 \\ 0.070 & 0.040 \\ 0.970 & 0.025 \\ \text { TOTAL } & 1.276 \\ E(K)=0.021 M E V & \\ \text { OH= } 1.320 & \\ \text { HPH }=1.32 & \end{array}$

$N(K)$
0.044
0.0
0.0
0.0
0.
0.
0.
0.
0.
0.0
0.044

$N(G) E(G)$
(MEV/D)
0.001
0.010
0.017
0.041
0.028
0.100
2.117
0.362
0.035
0.024
0.737
$X=0.001$
$E D=0.737 M E V / D$
$D=0.737 M E V / D$

$\begin{aligned} E D & =0.737 M E V / O \\ H E D & =0.737 M E V / D\end{aligned}$

SOURCE... NOS $61-4-15$

$\begin{array}{cl}E(G) & N(G) \\ \text { IMEV } & \\ 0.129 & 0.476 \\ \text { TOTAL } & 0.476 \\ E(K)=0.021 \text { MEV } & \\ \text { OH: } 1.000 & \\ \text { HOH }=1.000 & \end{array}$

$\begin{array}{lc}N(K) & N(G) E(G) \\ & (M E V / D) \\ 0.524 & 0.061 \\ 0.524 & 0.061 \\ & \text { PROOX }=0.011 \\ & E D=0.072 M E V / D \\ & \text { HED }=0.072 M E V / O\end{array}$

$E(B)$

(MEV)

$0.520 \quad 0.040$

$0.770 \quad 0.020$

$1.115 \quad 0.400$

$1.180 \quad 0.500$

$1.220 \quad 0.050$

105 RU 44

$4.40 \quad 4$
105 RHI 45 $38.00 \mathrm{~S}$

$E(B)$
(MEV)


SOURCE... NDS $6:-4-15$

105 RH2 45 $36.00 \mathrm{H}$

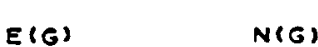

0.097

N(K)

$N(G) E(G)$

(MEV/O)

$E(B)$

(MEV)

0.560

$N(B)$

0.100

0.900

0.003

\section{$0000=0.000$}

$E D=0.031 M E V T O$

HED $=0.031$ MEV $/ O$

$O H=0.100$

$M O H=0.100$

\subsection{7} =.100

SOURCE... O.J. SEGAERT AND J.L. DEMUYNCK, MUCL. PHYS. 16, 49261960$)$

106 RH1 45

$N(a)$

0.400

0.380

0.110

0.100

1.180

.620

0.095

0.124

0.209

0.198

0.020

0.186

0.097

0.198

0.198

0.023

0.013

0.007

1.719

DRODX $=0$.

$E D=1.719 M E V / D$

HED $=1.719 M E V / D$ (

SOURCE... S.Y. AMBIYE AND R.P. SHARMA, NUCL. PHYS. 29,657 (1962).

106 RH2 45 $N(B)$

0.672

0.125

0.170

0.020

TOTAL

$\begin{array}{ll}E(G) & N(G) \\ \text { (MEV) } & \\ 0.513 & 0.315 \\ 0.624 & 0.139 \\ 0.860 & 0.005 \\ 1.060 & 0.019 \\ 1.140 & 0.006 \\ & 0.484 \\ E(K)=0.022 M E V & \\ P H=0.484 & \\ H P H=0.484 & \end{array}$

$N(K)$
0.
0.
0.
0.
0.
0.

N(G)E(G)

0.162

0.087

0.004

0.007

PROOX= 0 .

$E D=0.280 M E V / O$

ElB

3.550

3.050

2.000

$\mathrm{HPH}=0.484$

HED $=0.28$ OMEV O 
SOUREE... W.R. PIERSON, H.C. GRIFFIN AND C.D. CORYELL. PHYS. REV. 127. 1708 (1962).

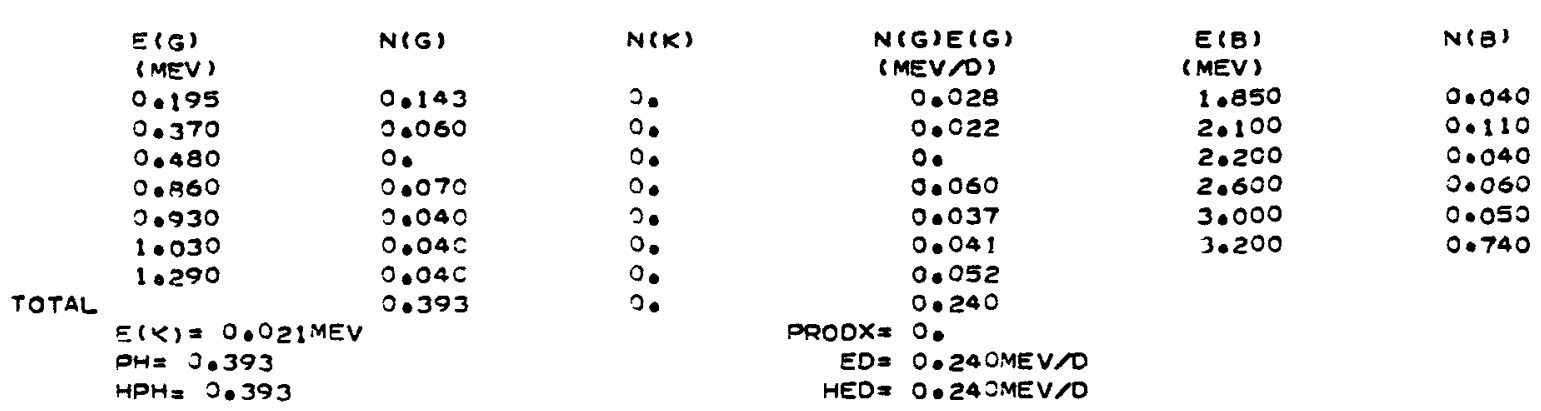

SOURCE... 'N.R. PIERSON, 4.C. GRIFFIN ANO C.D. CORYELL, PHYS. REV. 127, 1708 (1962)

$\begin{array}{ll}E(G) & N(G) \\ \text { (MEV) } & \\ 0.115 & 0.005 \\ 0.285 & 0.025 \\ 0.307 & 0.730 \\ 0.365 & 0.015 \\ 0.390 & 0.107 \\ 0.470 & 0.010 \\ 0.570 & 0.018 \\ 0.575 & 0.027 \\ \text { TOTAL } & 0.937 \\ E(K)=0.022 M E V & \\ \text { PH= } 0.937 & \\ H P H=0.937 & \end{array}$

$N(K)$
0.
$0:$
$0:$
0.
0.
$0:$
0.

ROOX $=0$.

$E D=0.312 M E V / D$

$\begin{array}{ll}E(B) & N(\theta) \\ \text { (MEV) } & \\ 0.830 & 0.070 \\ 0.930 & 0.020 \\ 1.100 & 0.080 \\ 1.200 & 0.710 \\ 1.510 & 0.170\end{array}$

107 RU 44

$4.60 \mathrm{M}$

SOURCE... W.R. PIERSON, H.C. GRIFFIN ANO C.D. CORYELL, PHYS. REV. 127, 1708 (1962)

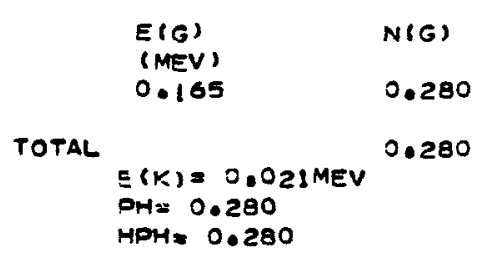

$N(K)$
0.

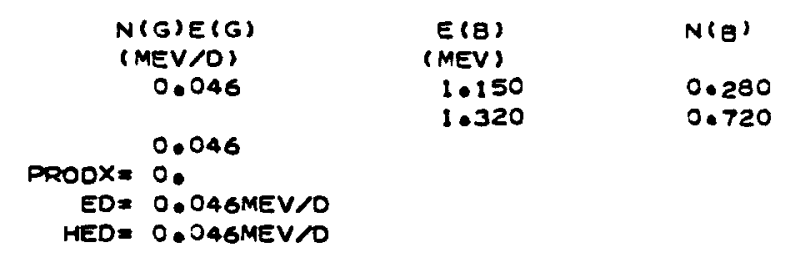

108 RU 44

$4.30 \mathrm{M}$

4.30 .9
107 RH2 45

$22.00 \mathrm{M}$ 
SOURCE... W.R. PIERSON, H.C. GRIFFIN ANO C.O. SORYELL, PHYS. REV. 127. 1708 (1962)

108 RH 45

$18.00 \mathrm{~S}$

$\begin{array}{cl}E(G) & N(G) \\ (M E V) & \\ 0.430 & 0.430 \\ 0.510 & 0.100 \\ 0.620 & 0.220 \\ 1.520 & 0.051 \\ \text { TOTAL } & 0.801 \\ E(K)=0.022 M E V & \\ \text { PH }=0.801 & \\ H P H=2.801 & \end{array}$

N(K)
0.
0.
0.
0.

$N(G) E(G)$
(MEV/O)
0.185
0.051
0.136
0.078
0.450

$E(B)$

N(B)

3.500

PRODX $=0$.

$E D=0.45 O M E V / D$

HED $=0.450 M E V / O$

SOURCE... VOS $60-2-47$

\begin{tabular}{|c|c|c|}
\hline & $\begin{array}{l}E(G) \\
(M E V)\end{array}$ & $N(G)$ \\
\hline & 0.110 & 1.000 \\
\hline JTAL & $\begin{array}{l}\equiv(K)=0.021 \text { MEV } \\
\text { DH }=1.020 \\
\text { YPH }=1.300\end{array}$ & 1.000 \\
\hline
\end{tabular}

SOURCE... NOS SO-2-47

$\stackrel{\omega}{\omega}$

\begin{tabular}{|c|c|c|}
\hline & $\begin{array}{l}E(G) \\
(Y E V)\end{array}$ & $N(G)$ \\
\hline & 0.180 & 0.625 \\
\hline TAL & $\begin{array}{l}E(K)=0.0224 E V \\
\text { OH= }=1.000 \\
H P M=1.000\end{array}$ & 2.625 \\
\hline
\end{tabular}

SOURCE... NOS $60-2-47$

$\begin{array}{ll}E(G) & N(G) \\ (M E V) & \\ 0.088 & 0.100 \\ \text { TOTAL } & 0.100 \\ E(K)=0.023 \text { HEV } & \\ \text { PHE } 1.000 & \\ \text { HPH }=1.000 & \end{array}$

SOURCE... NDS $60-2-47$

$\begin{array}{cc}E(G) & N(G) \\ \text { (MEV) } & \\ 0.088 & 0.046 \\ \text { TOTAL } & 0.046 \\ E(K)=0.023 M E V & \\ \text { PHI } 0.482 & \\ \text { MPH }=0.482 & \end{array}$

$\begin{array}{lc}\text { N(K) } & N(G) E(G) \\ & \text { (MEV/O) } \\ 3 . & 0.110 \\ 3 . & 0.110 \\ & \text { PRODX= } 0.11 \\ & E D=0.110 \text { MEV } / D \\ H E D= & 0.110 M E V / O\end{array}$

$\begin{array}{lc}N(K) & N(G) E(G) \\ & \text { (MEVIO) } \\ 0.375 & 0.112 \\ 0.375 & 0.112 \\ & \text { PRODX }=0.009 \\ & E D=0.121 \text { MEV } D \\ \text { HED }=0.121 \text { MEV } D\end{array}$

$\begin{array}{lc}N(X) & N(G) E(G) \\ & \text { (MEV/O) } \\ 0.900 & 0.009 \\ 0.900 & 0.009 \\ & \text { PRODX }=0.020 \\ & \text { ED }=0.029 \text { MEV } / O \\ & \text { HEO }=0.029 M E V / O\end{array}$

$\begin{array}{lc}N(K) & N(G) E(G) \\ & (M E V / O) \\ 0.436 & 0.004 \\ 0.436 & 0.004 \\ & \text { PRODX }=0.010 \\ & E D=0.014 M E V N O \\ & H E D=0.014 M E V / D\end{array}$

3.600

4.120

109 Rm! 45

$50.00 \mathrm{~s}$

$E(B)$

(MEV)

$N(B)$

0.050

0.170

0.170

109 PO1 46

$4.70 \mathrm{M}$

E(B)

N(B)

109 PD2 46

$14.00 \mathrm{H}$

$E(B) \quad N(B)$

(MEV)

$1.025 \quad 10000$

109 AGI 47

39.005
$E(8)$

(MEV)
N(B)

(2)


SOURCE... S.F. ECCLES, PHYSICA 28, 251 (1962).

\begin{tabular}{|c|c|c|c|c|}
\hline & $\begin{array}{l}E(G) \\
\text { (MEV) } \\
0.170\end{array}$ & $\begin{array}{l}N(G) \\
0.300 \\
0.300\end{array}$ & $\begin{array}{l}N(K) \\
0.380 \\
0.380\end{array}$ & $\begin{array}{c}\text { N(GIE(G) } \\
\text { (MEV D) } \\
0.051 \\
0.051\end{array}$ \\
\hline & $\begin{array}{l}E(K)=0.022 \mathrm{MEV} \\
P H=0.680 \\
H P H=0.680\end{array}$ & & & $\begin{aligned} \text { PRODX } & =0.008 \\
\text { ED } & 0.059 M E V / O \\
\text { HED } & =0.059 M E V / O\end{aligned}$ \\
\hline
\end{tabular}

$E(B)$

N(a)

$5.50 \mathrm{H}$

SOURCE... S.F. ECCLES, PHYSICA 28, 251 (1962).

11100246

$23.00 \mathrm{M}$

$\begin{array}{ll}E(G) & N(G) \\ (\text { MEV } & \\ 0.067 & 0.036 \\ 0.108 & 0.007 \\ 0.129 & 0.011 \\ 0.148 & 0.019 \\ 0.293 & 0.009 \\ 0.367 & 0.011 \\ 0.399 & 0.046 \\ 0.435 & 0.019 \\ 0.479 & 0.011 \\ 0.523 & 0.011 \\ 0.584 & 0.027 \\ 0.650 & 0.033 \\ 0.727 & 0.017 \\ 0.828 & 0.011 \\ 0.865 & 0.004 \\ 0.963 & 0.002 \\ 1.030 & 0.004 \\ 1.140 & 0.009 \\ 1.220 & 0.003 \\ 1.315 & 0.003 \\ 1.400 & 0.004 \\ 1.600 & 0.001 \\ 1.690 & 0.008 \\ 1.780 & 0.004 \\ 1.990 & 0.003 \\ E(K)=0.023 M E V & 0.317 \\ 0 H=0.317 & \\ H D H=0.317 & \end{array}$

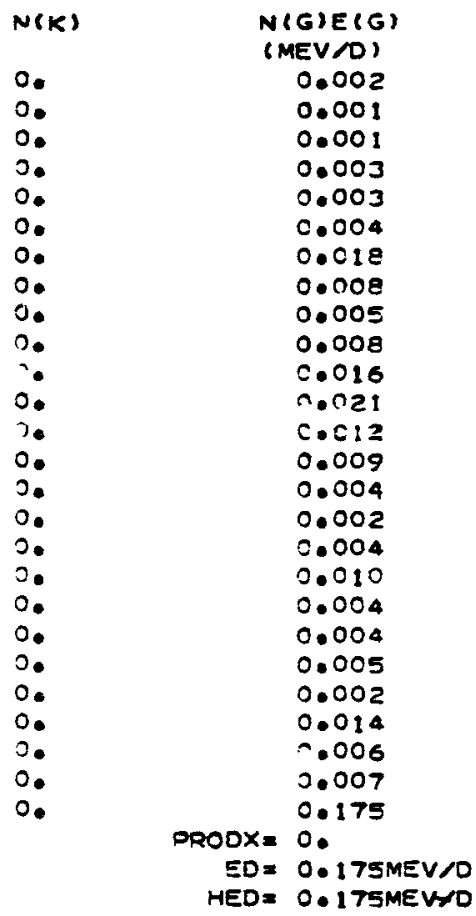

$E(8)$

$N(8)$

(MEV)

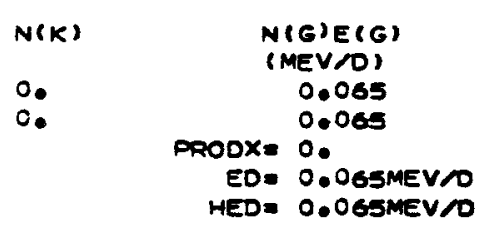

E(B)

(MEV) 
SOURCE... NOS 60-2-79

$\begin{array}{llll}111 & 162 & 47\end{array}$ 7.600

N(K)
0.001
0.001
0.0
0.002
TOTAL E(G) N(E) 0.060 0.072

$E(K)=0.024 M E V$

$\mathrm{PH}=0.074$

HPH: 0.074

SOURCE.. NOS $60-2-89$

$\begin{array}{ll}E(G) & N(G) \\ (M E V) & \\ 0.018 & 0.200 \\ \text { TOTAL } & 0.200 \\ \equiv(K)=0.023 M E V & \\ \text { SHE } 1.000 & \\ \text { MOH }=0.800 & \end{array}$

SOURCE... IDS 60-2-90 N(G)E (G)
(MEV/O) (MEVNO) 0.022 0.000

PROOX $=0.000$

$E D=0.023 M E V N O$

HED $=0.023 \mathrm{MEV} / D$

$E(B)$

n(E)

0.062

0.011

$0.69 n$

0.790

0.927
$112 \triangle G \quad 47$

$3.20 \mathrm{H}$
1120046

$21.00 \mathrm{H}$

$E(B)$

(MEV)

(MEV NO) (MEV)

$N(B)$

1.000

0.800

0.004

PROOX $=0.018$

$E D=0.022$ INEV/O

HED $=0.018 M E V / O$

N(G)ERG
SMEV/OI
0.002
0.001
0.001
0.014
0.017
0.018
0.018
0.011
0.068
0.025
0.015
0.014
0.013
0.052
0.012
0.074
0.007
0.014
0.007
0.018
0.031
0.246
0.0679
0.019

PRODX=0.

$E D=0.679 M E V / O$

HED $=0.679 M E V / O$

$N(B)$

$0.460 \quad 2.570$

$0.750 \quad 2.630$

0.920
1.220

$\begin{array}{ll}1.220 & 3.420 \\ 1.340 & 4.040\end{array}$

$\begin{array}{ll}1.340 & 4.040 \\ 1.500 & 0.018\end{array}$

$\begin{array}{ll}1.500 & 0.018 \\ 1.610 & 0.020\end{array}$

$1.780 \quad 0.044$

$2.010 \quad 0.053$

$2.200 \quad 0.006$

$2.570 \quad 0.016$

2.630

2.730

3.420

$\begin{array}{ll}3.420 & 0.180 \\ 4.040 & 0.560\end{array}$

$1=0.024 M E V$

$P H=0.696$ 
SOURCE... NOS $60-3-104$

$115 \operatorname{CO} 48$

E(G) N(G)

(MEV)

$\begin{array}{ll}0.485 & 0.023 \\ 1.300 & 0.010\end{array}$

$0.935 \quad 0.023$

TOTAL

0.036

$E(K)=0.025 \mathrm{MEV}$

$0.036 \quad c$

MPH $=0.036$

SOURCE... NDS $60-3-104$

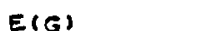

E(G)

2.240

0.523

0.263

0.033

0.490

0.005

TOTAL

0.260

0.006

0.017

$E(K)=0.025 M E V$

0.389

SOURCE... WOS $50-3-104$

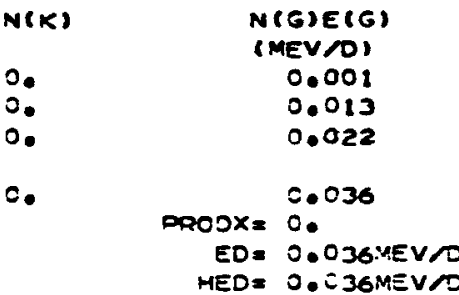

$E(B)$

$N(B)$

43.000

MED $=0.036 \mathrm{MEV} / \mathrm{O}$

$0.200 \quad 0.003$

$0.335 \quad 0.010$

$\begin{array}{ll}0.687 & 0.020 \\ 1.630 & 0.967\end{array}$

$\begin{array}{ll}E(G) & N(G) \\ (M E V) & \\ O .335 & 0.516 \\ \text { TOTAL } & 0.516 \\ E(K)=0.025 M E V & \\ \text { OH= } 0.877 & \\ \text { HOH }=0.877 & \end{array}$

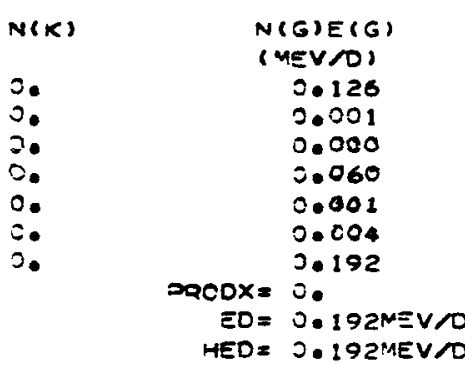

$E(B)$

(MEV)

0.590

0.630

0.850

1.110

1 is $\operatorname{CD} 48$ $53.00 \mathrm{H}$

N(e)

0.244

0.126

3.621

$E D=0.192 M E V / O$ HED $=0.192 M E V / O$

$4.51 H$

$\begin{array}{lc}N(K) & N(G) E(G) \\ & \text { (MEV/D) } \\ 0.361 & 0.173 \\ 0.361 & 0.173 \\ & \text { DRODX }=0.009 \\ & E D=0.182 M E V / D \\ & \text { HED }=0.182 \text { VEV }\end{array}$

$E(\xi)$

MEV)

$N(g)$

0.055

$\begin{aligned} E D & =0.182^{M E V} / D \\ \text { HED } & =0.182 \mathrm{MEV} / \mathrm{D}\end{aligned}$

SOURCE... NDS $60-3-117$

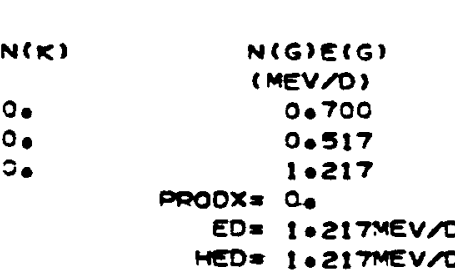

116 AG 47

$2.50 \mathrm{~m}$

\section{E(3)}

S.000

$N(B)$

1.000

$0.700 \quad 1.000$

2.000

PH= 2.000

HDH 2.000

HED = 1.21 TMEVIO 
SOYRCE... C.F. MILLER, USNDROL-TR-160, (1957).

$\begin{array}{ll}E(G) & N(G) \\ \text { (MEV) } & \\ 0.291 & 0.570 \\ 0.430 & 0.120 \\ 0.242 & 0.147 \\ 1.270 & 0.700 \\ 1.550 & 0.150 \\ \text { TOTAL } & 1.682 \\ E(<)=0.024 M E V & \\ 04=2.020 & \\ H 04=2.000 & \end{array}$

$N(K)$
0.280
0.030
0.010
0.
0.0
0.320

$N(G) E(G)$
(MEV/O)
0.160
0.052
0.118
0.889
0.232
1.451
PRODX $=0.008$
$E D=1.458 M E V / D$
HED $=1.458 M E V / D$

$\begin{array}{ll}E(B) & \\ \text { (MEV) } & \\ -0 . & \end{array}$

$117 \mathrm{CO1} 48$

$3.00 \mathrm{H}$

SOURCE... NOS $62-3-131$

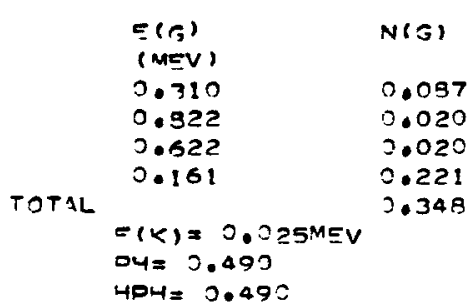

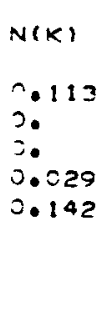

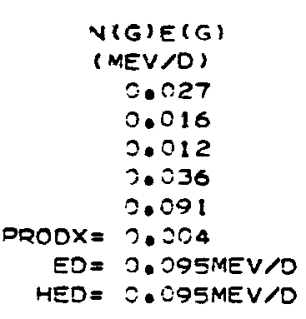

$E(3)$

(MEV)

0.950

1.620

$N \&=1$

117 IN1 49 $2.00 \mathrm{H}$

0.040

0.230
0.530

1.770

SOURCE... NDS 6:-3-131

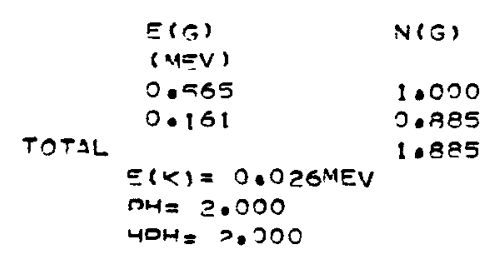

$N(K)$
0.
0.115
0.115

SOUPCE... VDS $60-3-131$

$N(G) E(G)$
$(M E V / D)$
0.565
0.142
0.707
PRODX $=0.003$
$E D=0.710$ MEV
HED $=0.710$ MEV/O

E(B)
(MEV)
0.740

117 IN2 49 $45.00 \mathrm{M}$

$N(B)$

1.000

$\begin{array}{cl}E(G) & N(G) \\ \text { (MEV) } & \\ 0.161 & 0.885 \\ 0.159 & 0.310 \\ \text { TOTAL } & 1.195 \\ E(K)=0.026 \mathrm{MEV} & \\ O H=2.000 & \\ \text { HOH }=2.000 & \end{array}$

$N(K)$
0.115
0.690
0.805

19 SNI 50

4.000 
SOURCE... NOS $62-3-142$

11 INZ $49^{\circ}$

5.005

$\begin{array}{cccc}\text { N(K. } & \text { N(G)E(G) } & \text { E(S) } & \text { NIE) } \\ \text { (MEVNO) } & \text { (MEV) } & 0.244 \\ 0 . & 3.300 & 0.220 \\ \text { O. } & 0.244 & 4.520 & 0.820\end{array}$

DROOX $=0$.

$E D=0.244 M E V / O$

HEO $=0.244$ MEVTO

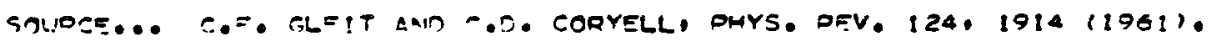

119 INI 49 18.004

$N(3)$

$0.4=0$

0.500

0.050

s.

0.017

\section{$000 x=0.052$}

ED $=0.053 M E V / O$

MED = 3.053 MEVIO

$N(K)$

$(B)$
(NEV)

2.750

2.720
1.820

(⿻)

w

Qur $=2.000$

HPHE 3.09 ?

0.073

SOURCE... C.E. GLEIT AND C.0. CORYELL, DHYS. REV. $124,1914(1961)$.

119 IN2 40 $2.50 "$
TOTAL

\begin{tabular}{|c|c|}
\hline $\begin{array}{l}E(\theta) \\
(n=v)\end{array}$ & $N(G)$ \\
\hline $\begin{array}{l}2.795 \\
2.731 \\
2.02 a\end{array}$ & $\begin{array}{l}0.950 \\
2.05 \\
0.050\end{array}$ \\
\hline
\end{tabular}

N(K)
$\therefore$ :.
O:

\section{$N(G) E(G)$}

(MEV D)

2.756
2.537

0.001

0.201

$D R O D X=0$.

$E D=0.794 M E V / O$

HED $=0.794 \mathrm{MEV} N$

SOURCE... C.E. GLEIT AND C.D. CORYELL, PHYS. REV. 124.1914 (1961).

$\begin{array}{ll}E(G) & N(G) \\ \text { (WEV) } & 0.120 \\ \text { O.N24 } & 0.120 \\ \text { TOTAL } & \\ \text { E(X) }=0.026 M E V & \\ \text { PHE } 1.000 & \\ \text { HOH }=1.000 & \end{array}$

$\begin{array}{lcr}N(K) & N(G) E(G) & E(B) \\ & (M E V / O) & \\ 0.880 & 0.003 & \\ \text { S.B8ก } & 0.003 & \\ & \text { (MEV) } \\ & P R O D X=0.023 \\ & E D=0.026 M E V / O \\ & H E D=0.026 M E V / O\end{array}$

245.000 
SOURCE... C.F, MILLER, USNROL-TR-160, (1957).

1200048

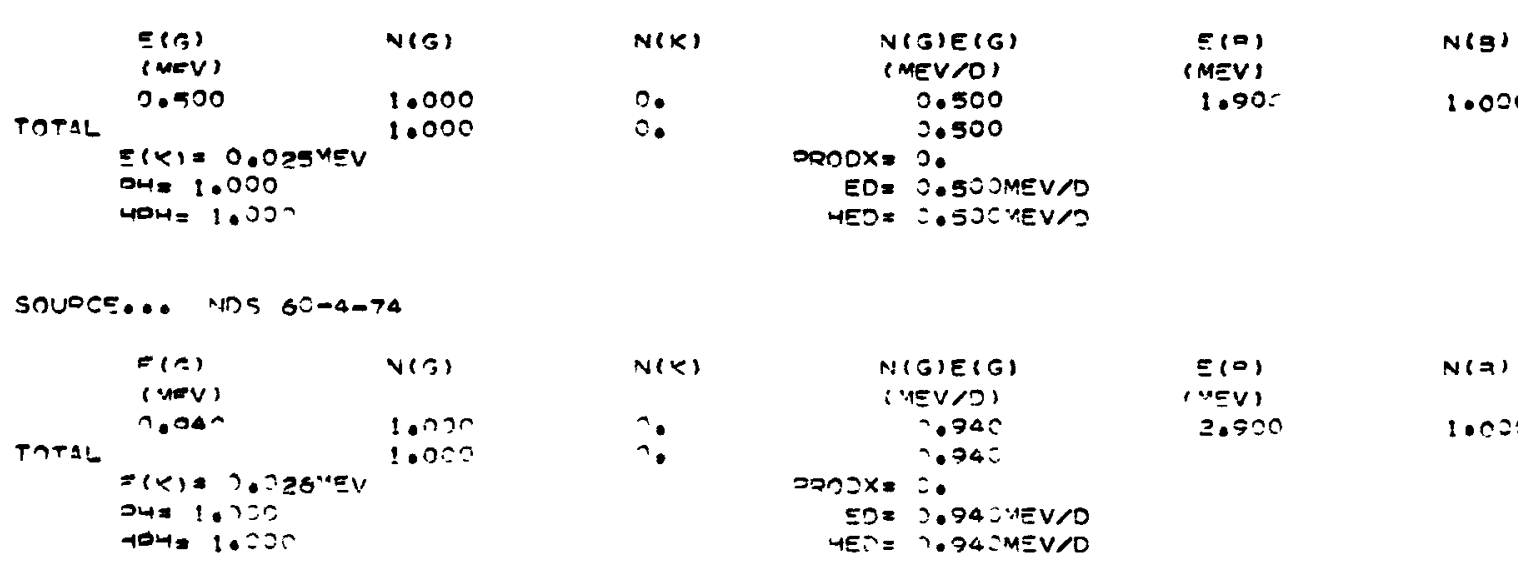

121 IN2 49 32.025

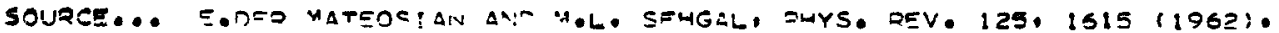

\begin{tabular}{|c|c|}
\hline POTAL & $\begin{array}{l}=(f) \\
(4, v) \\
n . n 75 \\
0 . n 51\end{array}$ \\
\hline & $\begin{array}{l}E(K)=2.3>7 M E V \\
\partial H=2.090 \\
\text { HM=2.วग? }\end{array}$ \\
\hline
\end{tabular}

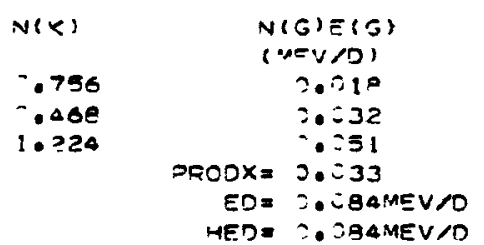

$\equiv(0)$

( $v=y)$

122 इ31 51 N(3) $3.52 \times$

SOURCE... vina 60-4-as

\begin{tabular}{|c|c|c|}
\hline & $\begin{array}{l}E(G) \\
(a n=V)\end{array}$ & $N(G)$ \\
\hline & $\begin{array}{l}0.564 \\
0.586 \\
1.258\end{array}$ & $\begin{array}{l}2.064 \\
3.023 \\
0.001\end{array}$ \\
\hline & $\begin{array}{l}E(K)=0.028 M E V \\
O H=0.671 \\
M O H=2.571\end{array}$ & $\therefore 668$ \\
\hline
\end{tabular}

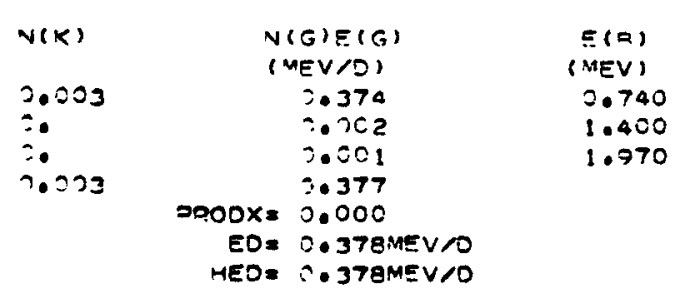

1225925 $2 \cdot 8=0$

SOURCE... NOS $60-6-23$

\begin{tabular}{|c|c|c|}
\hline & $\begin{array}{l}E(G) \\
(M V)\end{array}$ & $N(G)$ \\
\hline & 1.10 .0 & 1.000 \\
\hline & $\begin{array}{l}E(K)=0.026 \text { YEV } \\
\text { OH= } 1.050 \\
\text { MOM }=1.000\end{array}$ & \\
\hline
\end{tabular}

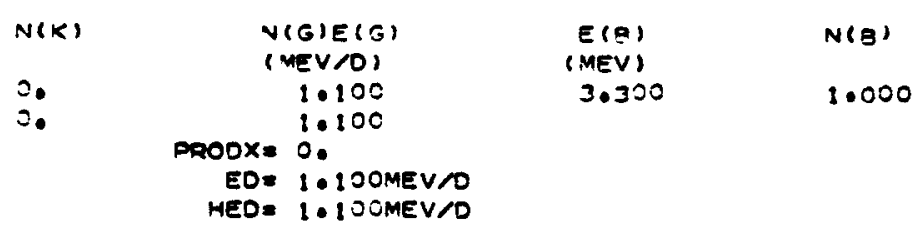

123 INZ 49 $10.00 \mathrm{~s}$ 
SOURCE... NDS 60-6-63

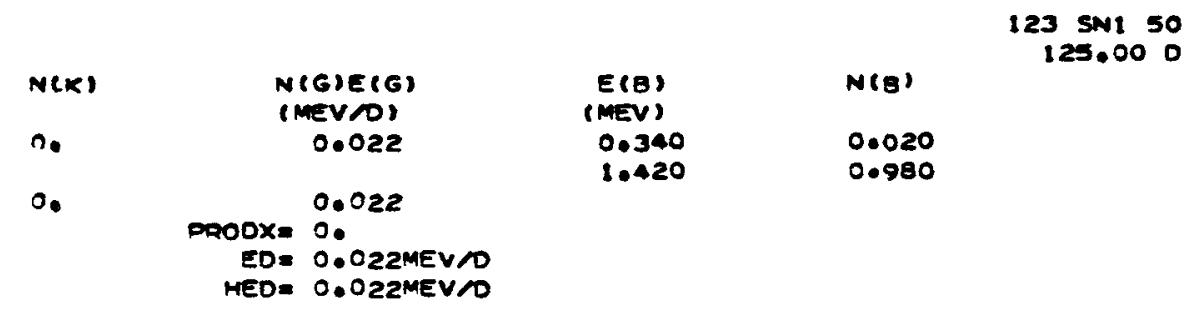

SOURCE... NDS 60-6-63

N(G)

0.020

TOTAL

E(K) $=0.027 M E V$
OME 0.020
MPH $=0.020$

E(G) N(G)

TOTAL

$\begin{array}{ll}\text { (MEV) } & \text { N(G) } \\ 0.160 & 0.885 \\ & 0.985\end{array}$

$E(K)=0.027 M E V$

DY. 1.000

HOH= 1.000

$\omega$

SOURCE... NOS 60-6-76

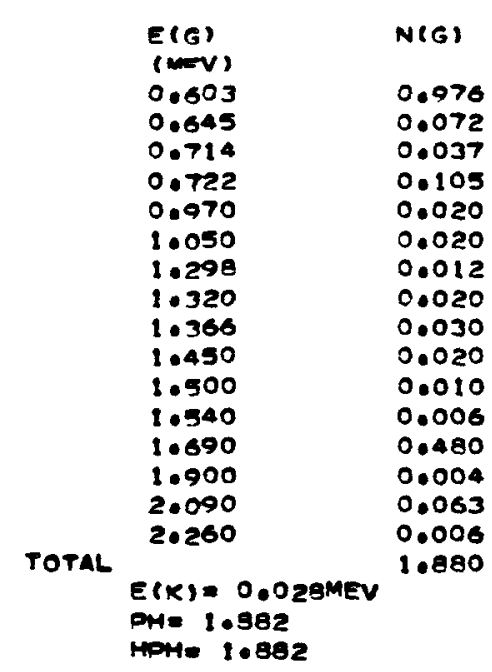

\begin{tabular}{|c|c|}
\hline$N(K)$ & $\begin{array}{l}N(G) E(G) \\
\text { (MEV }(D)\end{array}$ \\
\hline 0.115 & Q.14? \\
\hline$=.11=$ & $\begin{aligned} \text { FFOCX } & =0.142 \\
E D & =0.003 \\
\text { HED } & =0.145 \mathrm{MEV} / \mathrm{M} \\
& 0.145 \mathrm{M} V \mathrm{O}\end{aligned}$ \\
\hline
\end{tabular}

$E(\theta)$

(MEV)

1.250

N(B)

10000

123 SN2 50

$4: 00 \mathrm{M}$

12458251

60.000

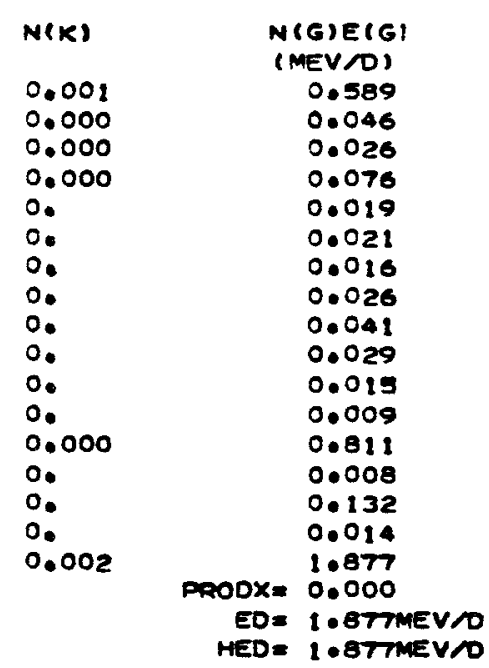

$E(B)$

$N(B)$

$0.051 \quad 0.020$

$0.225 \quad 0.110$

$0.621 \quad 0.510$

$0.954 \quad 0.050$

$\begin{array}{ll}0.954 & 0.050 \\ 1.016 & 0.015\end{array}$

$\begin{array}{ll}1.016 & 0.015 \\ 1.590 & 0.050\end{array}$

$2.313 \quad 0.230$ 
SOURCE... NOS 80-6-91

125 SN1 50

$9.76 \mathrm{M}$

$\begin{array}{ll}N(K) & N(G) E(G) \\ \text { (MEV/D) } & 0.325 \\ 0 . & 0.002 \\ 0 . & 0.003 \\ 0 . & 0.026 \\ 0 . & 0.356\end{array}$

PROOX $=0$.

ED=0.356MEV/O

HED $=0.356 \mathrm{MEV} / \mathrm{O}$

$\begin{array}{cl}E(\theta) & N(B) \\ \text { (MEV) } & \\ 0.650 & 0.022 \\ 2.040 & 0.978\end{array}$

0.978

$E(K)=0.027 M E V$

0.019

HOH= 1.022

SOURCE... NDS 60-6-91

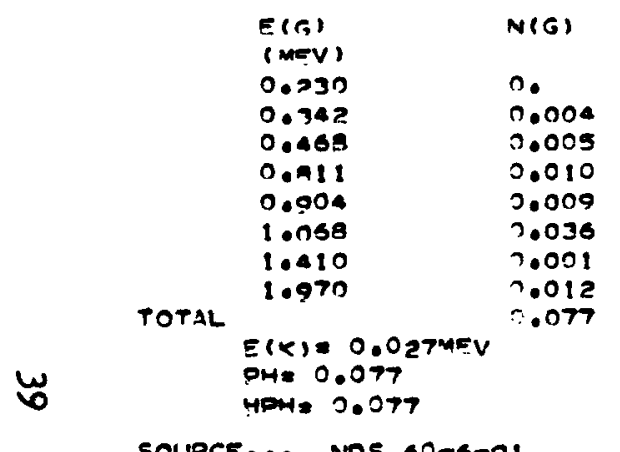

SOURCE... NOS 60-6-91

\begin{tabular}{|c|c|c|}
\hline & $\begin{array}{l}E(G) \\
\text { (MEV) }\end{array}$ & $N(s)$ \\
\hline & 0.035 & 0.064 \\
\hline & $\begin{array}{l}0.110 \\
0.109\end{array}$ & 0.040 \\
\hline & $\begin{array}{l}0.125 \\
0.171\end{array}$ & 0.003 \\
\hline & 0.176 & 0.068 \\
\hline & 0.203 & 3.002 \\
\hline & 0.306 & 0.003 \\
\hline & 0.255 & 0.015 \\
\hline & 0.290 & 0.015 \\
\hline & 2.389 & 0.008 \\
\hline & 0.379 & 3.012 \\
\hline & 0.427 & 0.310 \\
\hline & 0.462 & 0.100 \\
\hline & 0.598 & 0.190 \\
\hline & 0.604 & 0.052 \\
\hline & 0.633 & 0.110 \\
\hline & 0.652 & 0.007 \\
\hline & 0.668 & 0.016 \\
\hline & & 1.015 \\
\hline & $\begin{array}{l}E(K)=0.028 M E V \\
\text { OH= } 1.936 \\
\text { MOH }=1.936\end{array}$ & \\
\hline
\end{tabular}

$\begin{array}{lc}N(K) & N(G) E(G) \\ \text { (MEV/O) } \\ 0 . & 0.0 \\ 0 . & 0.001 \\ 3 . & 0.002 \\ 0.0 & 0.008 \\ 0 . & 0.008 \\ 0 . & 0.038 \\ 0 . & 0.001 \\ 0 . & 0.024 \\ 0 . & 0.083\end{array}$

PRODX $=0$.

$0.083 M E V / O$

HED $=0.083$ MEV $P$

$125 \mathrm{Se} 51$

$2.00 Y$

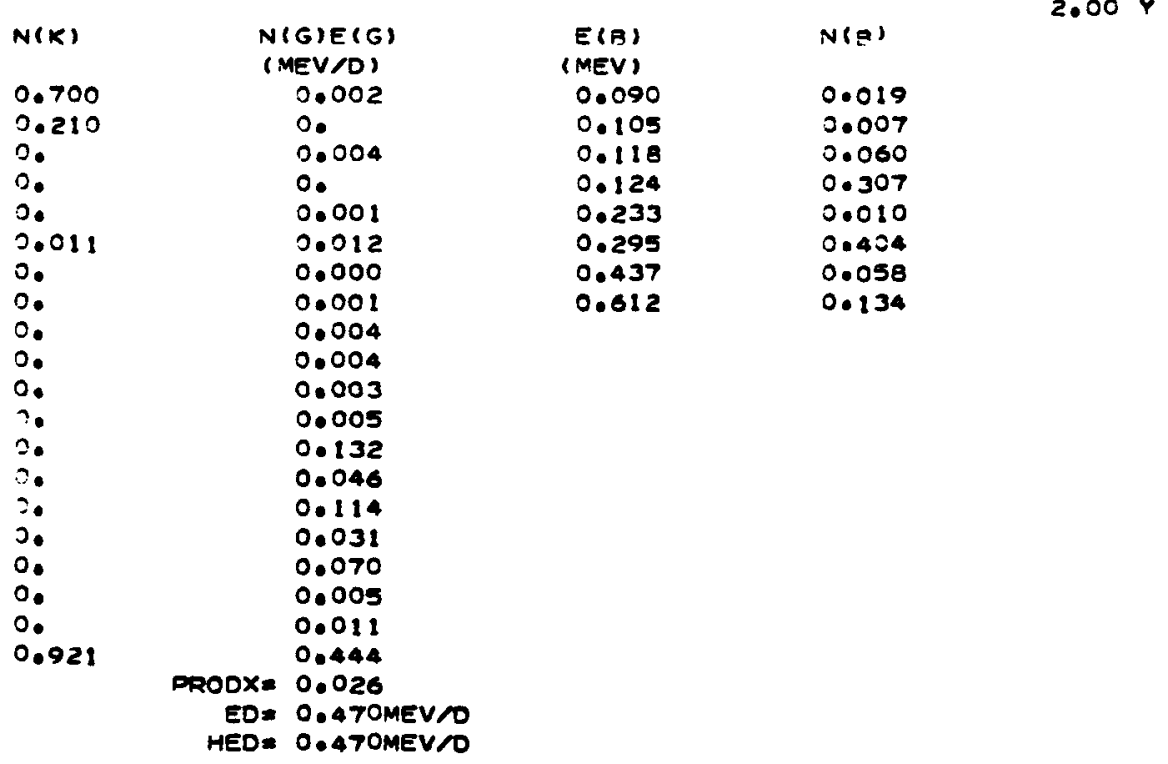


SOURCE... NDS SO S-9!

125 TEL 52

58.000

\begin{tabular}{|c|c|c|}
\hline & $\begin{array}{l}E(G) \\
(M=V)\end{array}$ & $N(G)$ \\
\hline & $\begin{array}{l}0.035 \\
0.110\end{array}$ & $\begin{array}{l}0.081 \\
0.006\end{array}$ \\
\hline 5146 & $\begin{array}{l}E(K)=0.02 \text { ?MEV } \\
\text { OH=2.000 } \\
\text { HOH=2.00? }\end{array}$ & \\
\hline
\end{tabular}

$\begin{array}{lc}N(K) & N(G) E(G) \\ & (M E V / D) \\ 0.919 & 0.003 \\ 2.994 & 0.001 \\ 1.913 & 0.004 \\ & \text { PRODX } 0.054 \\ & E D=0.057 \text { MEV NO } \\ & \text { HED }=0.057 M E V / D\end{array}$

$E(B)$

$N(\theta)$

(MEV

12658251

SOURCE... NIS SO-S-104

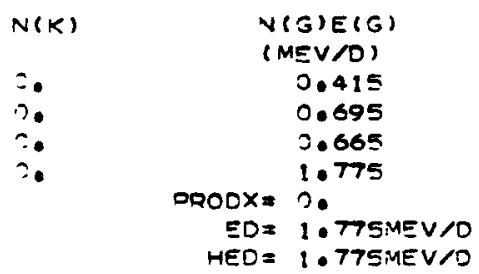

5 SOIRCE... C.F. MILLFR, USVRDL-TR-16), (1957).

\begin{tabular}{|c|c|c|}
\hline & $\begin{array}{l}E(G) \\
(\text { YeV })\end{array}$ & $N(G)$ \\
\hline & $\begin{array}{l}0.415 \\
0.495 \\
0.865\end{array}$ & $\begin{array}{l}1.000 \\
1.000 \\
1.000\end{array}$ \\
\hline & $\begin{array}{l}E(<)=0.02 \text { Q }^{M} E V \\
P H=3.050\end{array}$ & $3.00=$ \\
\hline
\end{tabular}

$H E D=1.775$ MEV $O D$

$$
\begin{aligned}
& \text { E(G) N(G) } \\
& 0.500 \quad 1.000 \\
& \text { TOTAL } \\
& 0.500 \\
& \begin{array}{l}
1.000 \\
1.000
\end{array} \\
& E(K)=2.027 \mathrm{MEV} \\
& 04=1.000 \\
& \text { पY⿱DH }=1.00 \text { ? }
\end{aligned}
$$

Nex
$?$.
$?$.

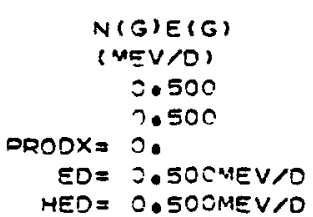

SOURCE... NDS $61-1-7 ?$

$\begin{array}{ll}\text { E(G) } & \text { N(G) } \\ \text { (MEV) } & \\ 0.06) & 0.040 \\ 0.248 & 0.140 \\ 0.310 & 0.060 \\ 0.463 & 0.540 \\ 0.772 & 0.240 \\ \text { TOTAL } & 1.020 \\ \text { E(K) } & 0.029 M E V \\ \text { OH= } 1.020 & \\ \text { HPH } 1.01 .020 & \end{array}$

N(x)
0.
0.
0.
0.
0.

$$
\begin{aligned}
& N(G) E(G) \\
& \text { (MEV/D) } \\
& 2.002 \\
& 0.035 \\
& 0.019 \\
& 0.250 \\
& 0.185 \\
& 0.491
\end{aligned}
$$$$
\text { PRODX }=0 .
$$

$E D=0.491 M E V T O$

HED $=0.491 \mathrm{MEV} / \mathrm{D}$
127 SN 50 $2.60 \mathrm{H}$ E (B)

3.000

N\{Q

1.000

$127 \mathrm{SB} \quad 51$ N(E)

$E(B)$

MEV)

$0.80 \mathrm{C}$

1.110

1.500

0.350

0.100

0.350

0.200 
SOURCE... NOS $61-1-72$

127 TEL 52

105.000

\begin{tabular}{|c|c|c|}
\hline & $\begin{array}{l}E(s) \\
(x-V)\end{array}$ & $N(s)$ \\
\hline & $\begin{array}{l}0.099 \\
0.059 \\
0.665\end{array}$ & $\begin{array}{l}0.985 \\
0.015 \\
0.000\end{array}$ \\
\hline & $\begin{array}{l}E(K)=0.029 \mathrm{VEV} \\
\text { OU=1.050 } \\
\text { HOU = } 1.000\end{array}$ & \\
\hline
\end{tabular}

NeK)
3.
$5:$
$0:$

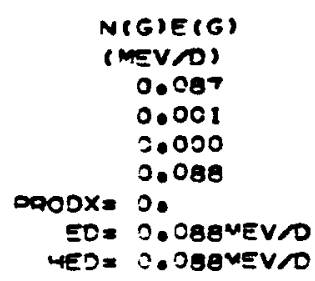

E(A)

(MEV)

N'B'

0.000

swure... vas 61-1-72

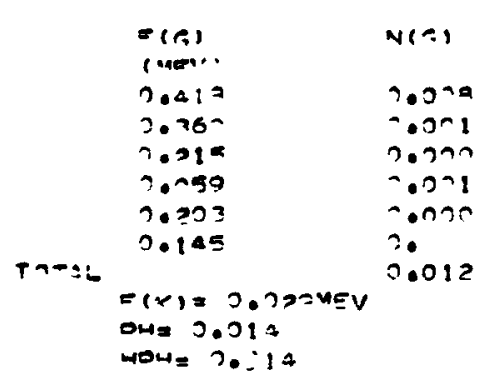

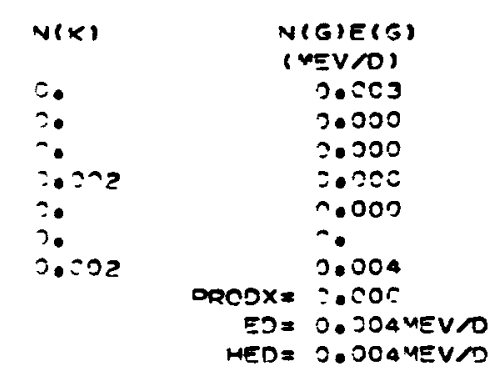

127 TE2 52 9.304

$E(a)$

(MEV)

0.270

0.695

N(S)

0.010 0.990

128 SN 50 $57.00 \mathrm{M}$

SOUPCE... O. DEL MAOMOL I I J. COLLARD, NUCL. OHYS. 36,109119621 .

\begin{tabular}{|c|c|c|}
\hline & $\begin{array}{l}E(3) \\
\text { (wev) }\end{array}$ & $N(G)$ \\
\hline & $\begin{array}{l}0.044 \\
0.072 \\
0.497 \\
0.570\end{array}$ & $\begin{array}{l}0.07 n \\
0.191 \\
0.50= \\
0.015\end{array}$ \\
\hline DTAL & $\begin{array}{l}E(x)=0.027 M E V \\
\text { OH= } 1.275 \\
\text { HOM }=1.275\end{array}$ & 1.025 \\
\hline
\end{tabular}

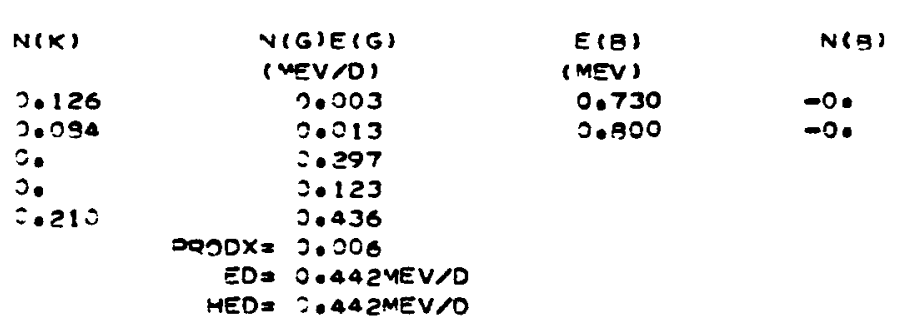

SOUPCE... D. DEL marmol and J. COLLARD. NUCL. PHYS. $36.109(1962)$.

12858152

$11.00 \mathrm{M}$

$\begin{array}{ll}E(T) & N(G) \\ \text { IMEV) } & \\ 0.320 & 0.910 \\ 0.754 & 1.960 \\ 1.074 & 0.040 \\ \text { TOTAL } & 2.810 \\ \text { EIKI) } 0.028 \text { NEV } & \\ \text { OH= } 2.010 & \\ \text { HOH }=2.810 & \end{array}$

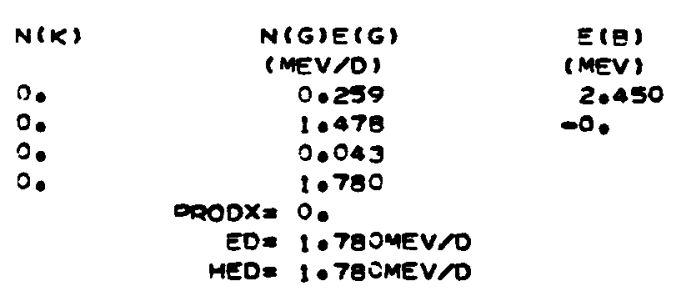

\author{
$N(B)$ \\ 0.850 \\ 0.150
}


sounce... NOS $61-1-93$

$120 \quad 153$

25.00

\begin{tabular}{|c|c|c|}
\hline & $\begin{array}{l}E(G) \\
(E-V)\end{array}$ & $N(G)$ \\
\hline & $\begin{array}{l}0.445 \\
0.750 \\
0.540 \\
0.990\end{array}$ & $\begin{array}{l}0.172 \\
0.002 \\
0.017 \\
0.003\end{array}$ \\
\hline & $\begin{array}{l}E(K)=0.030 \mathrm{MeV} \\
\text { OM= } 0.194\end{array}$ & \\
\hline
\end{tabular}

Nex)
0.
0.
0.
0.

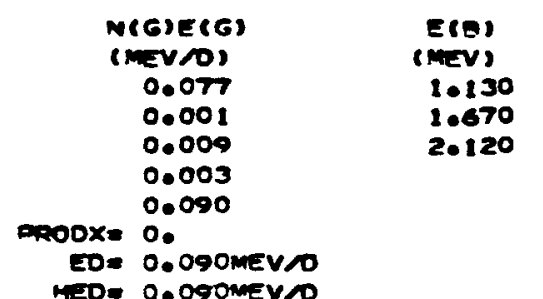

N(E)

0.020

$0.15=$

0.760

ED= 0.090MEV/O

SOURCE... C.F. MILLER, USVONL-TR-160. (1957).

$\begin{array}{cl}E(E) & N(S) \\ i(E V) & 0.590 \\ 2.155 & 0.550 \\ 2.309 & 1.000 \\ 0.534 & 0.250 \\ 0.799 & 2.390 \\ \text { TOTAL } & \\ E(K)=0.029 M E V & \\ P H=2.550 & \\ H P H=2.550 & \end{array}$

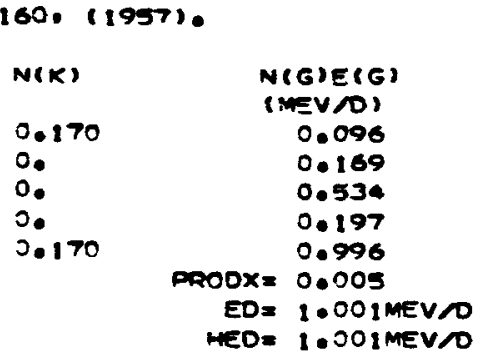

$\begin{array}{ll} & \\ \text { E(E) } & N(8) \\ \text { (MEV) } & \\ 1.250 & 0.250 \\ 1.560 & 0.550 \\ 1.870 & 0.200\end{array}$

$12958 \quad 51$ $4.60 \mathrm{H}$

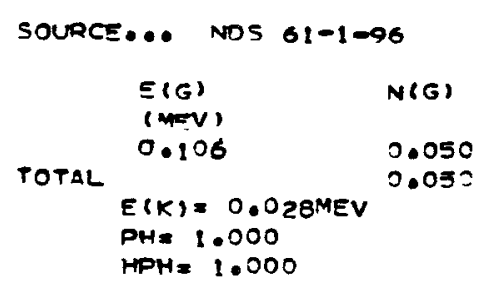

$\begin{array}{cc}N(K) & N(G) E(G) \\ & \text { (MEVND) } \\ 0.950 & 0.005 \\ 0.950 & 0.005 \\ & \text { PRODX }=0.027 \\ & E D=0.032 M E V / O \\ & \text { HED }=0.032 M E V / D\end{array}$

SOURCE... M.E. GRAVES AND A.C.G. MitChELL, PhYS. REV. 101, 701 (1956).

$N(B)$

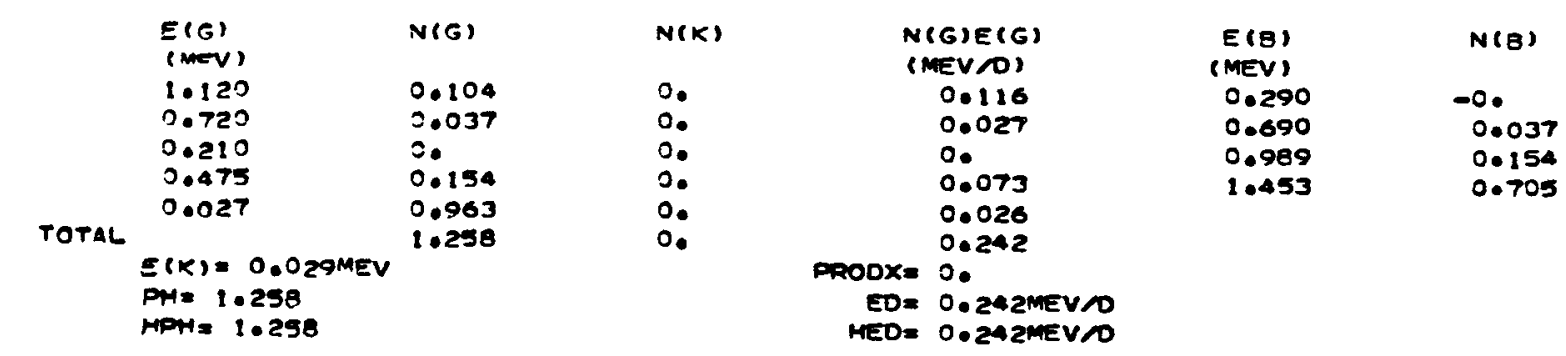

129 TEI 52 37.000

MED $=0.242 M E V O$ 
SOURCE... NOS $61-1-96$

\begin{tabular}{|c|c|c|}
\hline & $\begin{array}{l}\text { E(C) } \\
(\text { NEV })\end{array}$ & $N(s)$ \\
\hline & $\begin{array}{l}0.196 \\
3.042\end{array}$ & $\begin{array}{l}0.083 \\
0.1119 \\
0.201\end{array}$ \\
\hline & $\begin{array}{l}=(K)=3.030 \mathrm{MEV} \\
\mathrm{OH}=2.000\end{array}$ & \\
\hline
\end{tabular}

$\begin{array}{lc}N(K) & N(G) E(G) \\ & \text { (MEV/D) } \\ 0.917 & 0.016 \\ 0.882 & 0.005 \\ 1.799 & 0.021\end{array}$

PRODX $=0.055$

$E D=0.076 M E V / D$

$P=2.000$

HED $=0.076 \mathrm{MEV} / \mathrm{O}$

SOURCE... NDS $57-3-52$

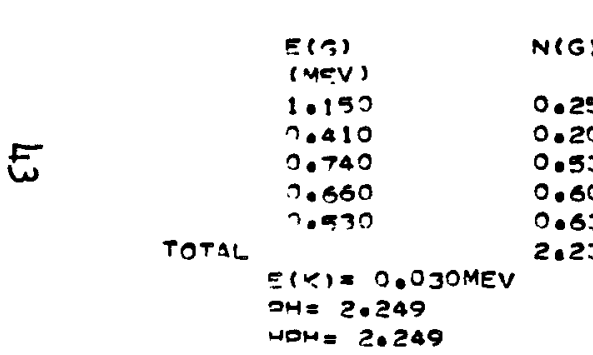

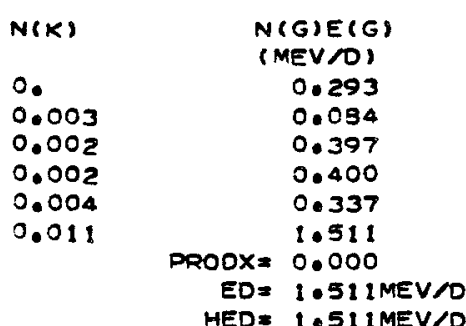

SOUDE=... C.F. MILLER, USNRDL-TR-160, (1957).

\begin{tabular}{|c|c|c|}
\hline & $\begin{array}{l}E(\sigma) \\
(M E V) \\
2.500\end{array}$ & $\begin{array}{l}N(G) \\
1.000\end{array}$ \\
\hline & $\begin{array}{l}E(K)=0.028 M E V \\
O H=1.000 \\
\text { MPH }=1.000\end{array}$ & \\
\hline
\end{tabular}

$\begin{array}{lc}\text { N(K) } & N(G) E(G) \\ 0 . & \text { (MEV/D) } \\ 0.600 \\ 0 . & 0.600 \\ & \text { PROOX }=0 . \\ & E D=0.600 M E V / O \\ & \text { HED }=0.600 \mathrm{MEV} / 0\end{array}$

$E(3)$

(MEV)

3.000

$E(B)$

0.600

0.600
1.020

1.020

$129 \times E 154$ 0.000

E(B)
MEV)

$N(B)$

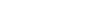

$130 \quad 53$

$N(5)$

0.470

0.530

0.002

HED $=0.600 \mathrm{MEV} / \mathrm{O}$ 
SOURCE... NOS $61-2-48$

131 TEI 52

$30.00 \mathrm{M}$

E TOTAL

0.020

$0.780 \quad 3.800$

0.182

0.

2.241

$E(K)=0.029 M E V$

PH= 2.613

SOURCE... NDS $61-2-48$

$\begin{array}{ll}E(G) & N(G) \\ \text { (MEV) } & \\ 1.130 & 0.100 \\ 0.985 & 0.1050 \\ 0.920 & 0.050 \\ 0.600 & 0.050 \\ 0.454 & 0.200 \\ 0.145 & 0.800 \\ \text { TOTAL } & 1.200 \\ \text { E(K) } & 0.029 M E V \\ \text { OHE } 1.408 & \\ \text { HPH } 1.408 & \end{array}$

$E(8)$

(MEV)

0.215

0.420

0.570

0.013

0.080

0.207

0.054

0.003

0.038

0.008

0.016

0.325

0.054

0.049

0.336

0.046

0.046

D200X $=0.011$

$E D=1.872 M E V / O$

HED $=1.872 M E V / D$

$N(K)$
0.
0.
0.
0.
0.
0.208
0.208

n(E)

0.036

0.430

0.038

$$
0.001
$$$$
0.190
$$

He

$N(G) E(G)$
(MEV/D)
0.113
0.
0.046
0.030
0.091
0.116
0.396
PRODX $=0.006$
ED= 0.402 MEV/O
HED $=0.402 M E V / O$

131 TE2 52

$N(8)$

E(B)

1. 150

1.150
1.360

1.680
2.140

0.100

0.050

0.250
0.600 
SOURCE... NDS $61-2-49$

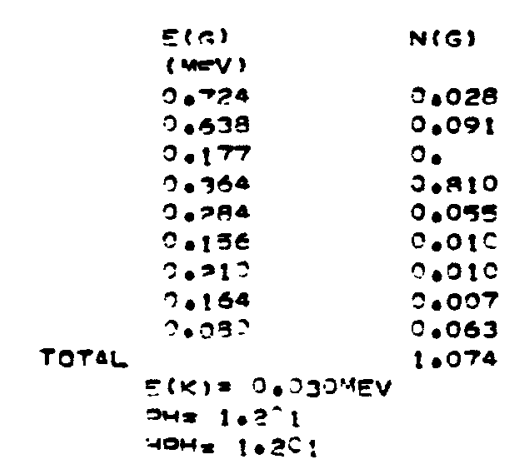

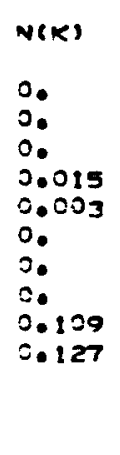

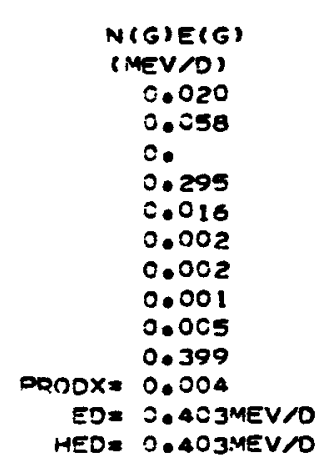

E(9)

(MEV)

0.250

0.330

0.606

0.810

$131 \operatorname{los}_{800}^{50}$

E

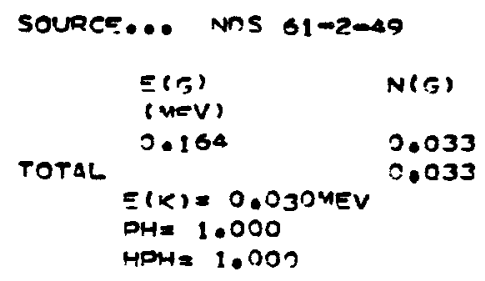

$N(K)$
0.967
0.967

$N(G) E(G)$
(MEV/D)
0.005
0.005
PRODX= 0.029
ED= 0.035 MEV/O
HED $=0.035$ MEV D

$E(g)$

(MEV)

131 XeI 54

12.000

SOURE... NDS $61-2069$

$\begin{array}{ll}E(G) & N(G) \\ (W E V) & \\ 0.233 & 0.935 \\ 0.052 & 0.160 \\ \text { TOTAL } & 1.095 \\ E(K)=0.029 M E V & \\ \text { OHE } 2.000 & \\ \text { MOH }=2.000 & \end{array}$

$N(x)$
0.055
0.840
0.905

$N(G) E(G)$
(MEV/D)
0.218
0.008
0.226
PROOX= 0.026
ED= $0.253 M E V / D$
HED $=0.253 M E V / O$
$E(3)$
(MEV)

N(B)

0.028

0.091

0.875

0.007

MOH $=2.000$

HED $=0.253 M E V N$ 
SOURCE... NDS 61-2-69

$132 \stackrel{53}{2.30}$

$\begin{array}{ll}E(G) & N(G) \\ (M E V) & \\ 0.240 & 0.013 \\ 0.375 & 0.046 \\ 0.460 & 0.022 \\ 0.523 & 0.190 \\ 0.624 & 0.052 \\ 0.647 & 0.090 \\ 0.650 & 0.170 \\ 0.670 & 0.990 \\ 0.720 & 0.065 \\ 0.775 & 0.820 \\ 0.950 & 0.190 \\ 1.142 & 0.045 \\ 1.300 & 0.031 \\ 1.400 & 0.083 \\ 1.450 & 0.014 \\ 1.500 & 0.018 \\ 1.650 & 0.007 \\ 1.750 & 0.004 \\ 1.919 & 0.009 \\ 1.990 & 0.010 \\ 2.370 & 0.007 \\ 2.180 & 0.002 \\ 2.352 & 0.001 \\ 2.420 & 0.001 \\ 2.540 & 0.001 \\ 2.530 & 0.000 \\ 2.300 & 0.000 \\ 150 & 2.882\end{array}$

$E(K)=0.030 \mathrm{MEV}$

$P H=2.882$

HPH $=2.832$

SOUPCE... NOS $61-2-36$

$N(K)$
0.
$0:$
$0:$
$0:$
$0:$
$0:$
$0:$
$0:$
$0:$
$0:$
$0:$
$0:$
$0:$
$0:$
$0:$
$0:$
$0:$
$0:$
$0:$
$0:$
$0:$
$0:$
$0:$
$0:$
$0:$
$0:$
$0:$
$0:$

$N(G) E(G)$

(MEV/O)

0.003

2.017

0.099

0.032

0.032

0.058

0.110

0.047

0.635

0.180

0.051

0.040

0.116

0.020

0.027

0.012

0.007

0.017

0.014

0.004

0.003

0.004

0.002

0.001

0.000

DROOX $=0$.

.197

$E D=2.197 \mathrm{MEV} / \mathrm{O}$

HEO $=2.197 \mathrm{MEV} / \mathrm{O}$

$E(P)$

$N(B)$

0.800

0.800
1.040

1.040

1.220

1.490

2.140

2.200

2.920

0.210

0.150

0.120

2.120

0.210

0.180

2.000

$\begin{array}{cl}E(G) & N(G) \\ (Y E V) & \\ 0.334 & 0.041 \\ 0.309 & 0.207 \\ 0.387 & 0.057 \\ 0.432 & 0.500 \\ 0.468 & 0.220 \\ 0.557 & 0.350 \\ 0.632 & 0.180 \\ 0.698 & 0.240 \\ 0.754 & 0.850 \\ 0.910 & 0.570 \\ 0.973 & 0.100 \\ \text { TOTAL } & 3.315 \\ \text { E(K) }=0.028 M E V & \\ \text { PHI } 3.404 & \\ \text { HPH }=3.404 & \end{array}$

$N(K)$
0.089
0.
0.
0.
0.
0.
0.
0.
0.
0.
0.
0.089

N(G)E(G)

( MEV DO)

0.014

0.064

0.022

0.216

0.103

0.195

0.114

0.168

0.641

0.519

0.097

2.152

PRODX $=0.002$

$E D=2.154 M E V / D$

133 TE1 52

$52.00 \mathrm{M}$

HED $=2.154 M E V / D$

(MEV)

1.300

2.400

$N(8)$

0.720

0.170 
SOURCE... C.F. MILLER, USNROL-TR-160, (1957).

133 TE2 52

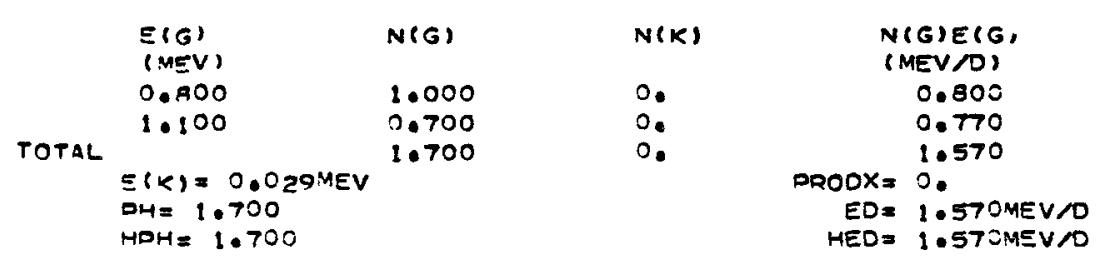

SMIJRCE... C.F. MILLER, USNROL-TD-160, $(1957)$.

$\begin{array}{ll}E(G) & N(G) \\ (M E V) & \\ 0.530 & 0.980 \\ 0.050 & 0.050 \\ 1.380 & 0.020 \\ \text { TOTAL } & 1.050 \\ E(K)=0.030 \mathrm{NEV} & \\ \text { OH }=1.050 & \\ \text { HOH }=1.050 & \end{array}$

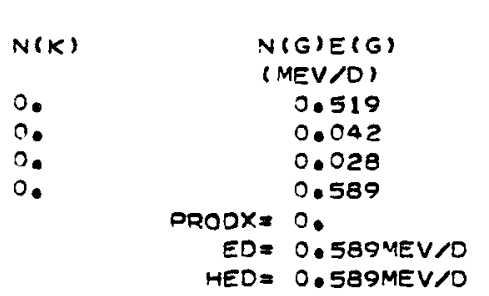

$\Xi$

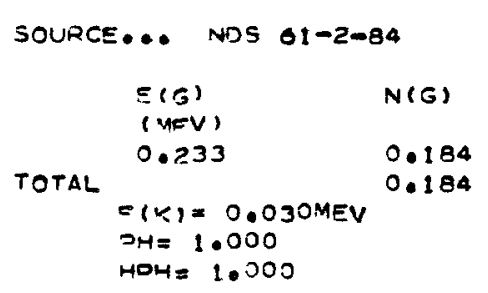

SOURCE... NDS $61-2-84$

$\begin{array}{cl}E(G) & N(G) \\ (M E V) & \\ 0.390 & 0 . \\ 0.302 & 0.000 \\ 0.160 & 0.001 \\ 0.079 & 0.008 \\ 0.081 & 0.410 \\ \text { TOTAL } & 0.419 \\ E(K)=0.032 M E V & \\ D H=1.009 & \\ H P H=1.009 & \end{array}$

$\begin{array}{lc}N(K) & N(G) E(G) \\ 0.816 & (M E V / D) \\ 0.816 & 0.043 \\ & 0.043 \\ & \text { PRODX }=0.025 \\ & E D=0.068 \text { MEV } 10 \\ \text { HED }=0.068 M E V / O\end{array}$

$E(B)$

$E(B)$

0.490

1.340

N(g)

0.070

0.930

$133 \div 53$

$20.80 \mathrm{H}$
$133 \times E 154$
2.300

$N(B)$

$\begin{array}{lc}N(K) & N(G) E(G) \\ & (M E V / O) \\ 0 . & 0.0 \\ 0 . & 0.000 \\ 0 . & 0.000 \\ 0.0 & 0.001 \\ 0.590 & 0.033 \\ 0.590 & 0.034 \\ & \text { PRODX }=0.019 \\ & E D=0.053 M E V / O \\ & \text { HED }=0.053 M E V / D\end{array}$

E(B)
(MEV)
0.046
0.268
0.347

$133 \times E 254$

$N(a)$

0.000

0.001

0.990 
SOURCE... C.F. MILLER, USNROL-TR-160, (1957).

134 TE 52

$\begin{array}{cc}E(G) & N(G) \\ \text { (MEV) } & \\ 1.500 & 10000 \\ \text { TOTAL } & 1.000 \\ E(K)=0.292 M E V & \\ P H=1.000 & \\ \text { MPHE } 10000 & \end{array}$

N(K)

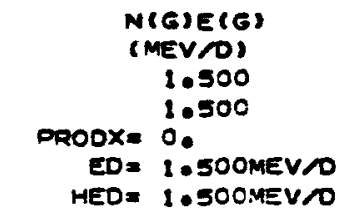
E(8)
(MEV)

$N(B)$
o.
HED $=1$. SOOMEV 0

SOURCE... vOS 61-2-97

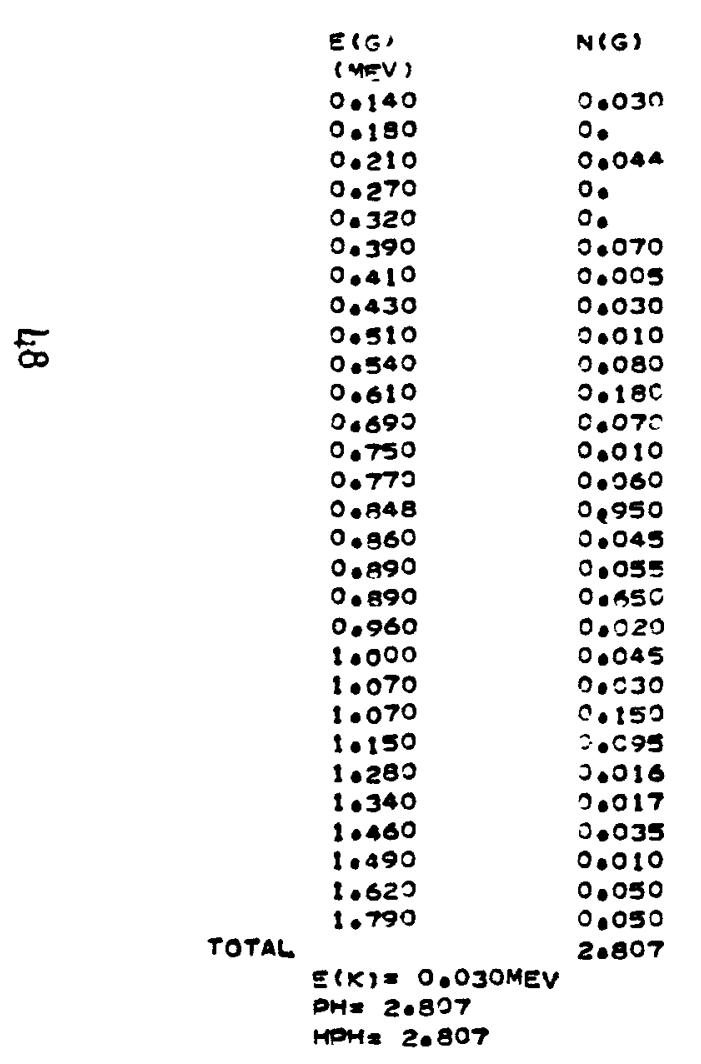

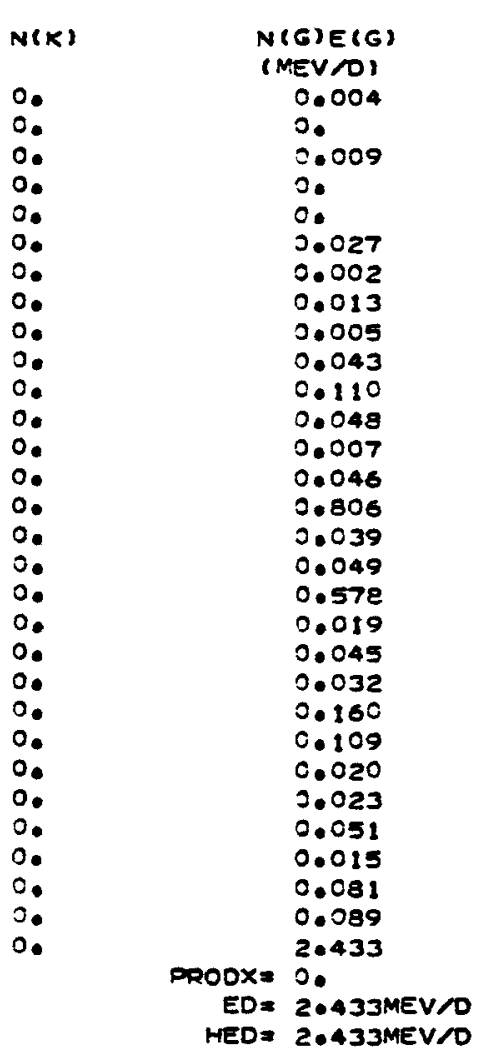

$\begin{array}{ll}E(B) & N(B) \\ (M E V) & \\ 0.500 & 0.065 \\ 1.050 & 0.010 \\ 1.250 & 0.230 \\ 1.490 & 0.150 \\ 1.680 & 0.075 \\ 1.810 & 0.095 \\ 2.210 & 0.120 \\ 2.410 & 0.250\end{array}$

$343^{1} 53$

.065

0.230

0.075

0.120

0.250 
SOURCE... NDS $81-2-97$

\begin{tabular}{|c|c|c|}
\hline & $\begin{array}{l}\text { E(G) } \\
\text { (NEV) }\end{array}$ & $N(G)$ \\
\hline & $\begin{array}{l}0.127 \\
0.137 \\
0.010\end{array}$ & $\begin{array}{l}0.682 \\
2.008 \\
0.0\end{array}$ \\
\hline DTAI & $\begin{array}{l}\Sigma(k)=0.033 \text { YEV } \\
D H=1.992 \\
\mathrm{HOH}=1.992\end{array}$ & 0.590 \\
\hline
\end{tabular}

$\begin{array}{lc}N(K) & N(G) E(G) \\ & (N E V / D) \\ 0.310 & 0.087 \\ 0.0 & 0.001 \\ 0.992 & 0.00 \\ 1.302 & 0.088 \\ & \text { DRODX }=0.043 \\ & E D=0.131 \mathrm{MEV} / 0 \\ & \text { HEO }=0.131 \mathrm{MEV} / \mathrm{C}\end{array}$

SOUQCE... NOS S1-2-97

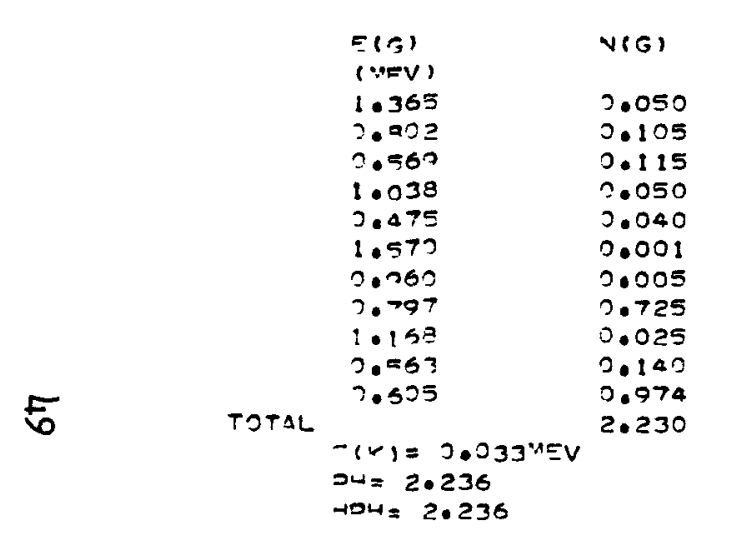

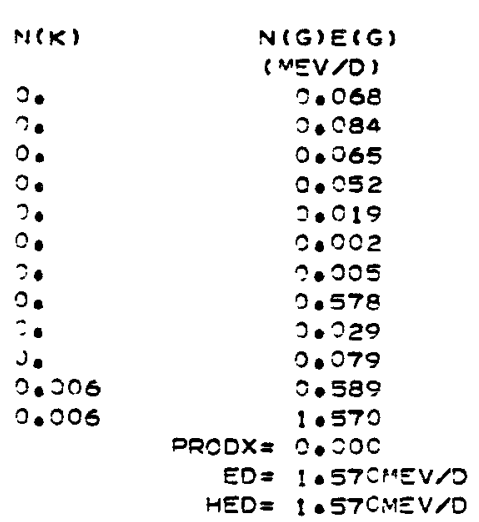

134 es2 55

$E(E)$

(MEV)

0.282

0.282
$0.41 C$

0.658

0.892

1.453

N(S)

0.010

34 CS1 55

.

(MEV I
0.550

10.010

SOUQCE... NOS $61-2-113$, NOS $6:-2-116$

$\begin{array}{ll}E(G) & N(G) \\ (M E V) & \\ 2.420 & 0.144 \\ 1.533 & 0.277 \\ 2.620 & 0.416 \\ 0.700 & 0.194 \\ 3.851 & 0.116 \\ 1.040 & 0.198 \\ 1.140 & 0.532 \\ 1.270 & 3.554 \\ 1.470 & 0.198 \\ 1.690 & 0.256 \\ 1.800 & 0.183 \\ \text { TOTAL } & 3.068 \\ \text { ERK } & \\ \text { DH= } 3.06030 & \\ \text { HPH }=3.068 & \end{array}$

$v(k)$
0.
$0:$
$0:$
$0:$
$0:$
$0:$
$0:$
$0:$
$0:$
$0:$
$0:$

$N(G) E(G)$
$(M E V / D)$
0.060
0.148
0.258
0.136
0.099
0.226
0.606
0.724
0.291
0.433
0.329
3.270
PQODX= 0.2
ED= $3.270 M E V / D$
HED $=3.270 M E V / D$

$\begin{array}{ll}E(B) & N(B) \\ (M E V) & \\ 0.470 & 0.035 \\ 1.000 & 0.040 \\ 1.400 & 0.250\end{array}$

135.60 .53

0.035

0.250 
SOURCE... NOS $61-2-113$

$135 \times E t$ SA

15.30

$\begin{array}{cc}E(G) & N(G) \\ \text { (MEV } & \\ O . S 28 & 0.830 \\ \text { TOTAL } & 0.830 \\ E(K)=0.030 \mathrm{YEV} & \\ \text { OHE } 1.000 & \\ \text { HPH }=1.000 & \end{array}$

SOURCE... NOS $01-2-113$

$\begin{array}{lc}N(K) & N(G) E(G) \\ & (M E V / D) \\ 0.170 & 0.438 \\ 0.170 & 0.438 \\ & \text { ORODX= } 0.005 \\ & E D=0.443 M E V / O \\ & H E D=0.443 M E V / O\end{array}$

$\begin{array}{cl}E(G) & N(G) \\ \text { (MEV) } & \\ 0.604 & 0.030 \\ 0.360 & 0.001 \\ 0.250 & 0.970 \\ \text { TOTAL } & 1.001 \\ \text { E(K) } & 0.032 M E V \\ \text { PHE } 1.053 & \\ \text { HPH }=1.053 & \end{array}$

SOURCE... NOS $61-2-122$

$\begin{array}{cl}E(G) & N(G) \\ (M E V) & \\ 1.000 & 0.060 \\ 2.400 & 0.120 \\ 0.710 & 0.030 \\ 2.250 & 0.070 \\ 3.200 & 0.050 \\ 1.910 & 0.050 \\ 0.390 & 0.190 \\ 1.720 & 0.020 \\ 2.840 & 0.030 \\ 1.350 & 0.040 \\ 0.200 & 0.120 \\ 2.630 & 0.110 \\ 1.320 & 0.950 \\ \text { EIKI) } & 1.89030 \text { MEV } \\ \text { PHTAL } & \\ \text { PHE } 1.890 & \\ \text { MPH. } 1.890 & \end{array}$

135 XE2 S4 $9.20 \mathrm{H}$

N(K)
0.
0.
0.052
0.052

N(G)E(G)

(MEV D)

0.018

0.000

0.242

DROOX $=0.261$

$E D=0.263 M E V / D$

HED $=0.263 \mathrm{MEV} / \mathrm{O}$

$E(B)$

(MEV)

0.910

$N(8)$

0.030

0.970

$136 \quad 53$

$93.00 \mathrm{~s}$
E(B)

Nie\}

$N(G) E(G)$
(MEV/D)
0.060
0.288
0.021
0.157
0.160
0.095
0.074
0.034
0.227
0.062
0.024
0.289
1.254
2.747
E.
$2.747 M E V / O$
PROD
$2.747 M E V / O$

(MEV

2.730

4.160

4.370

5.600

7.000

0.060

0.050

0.240

0.150

0.060
HED $=2.747 M E V / D$ 
SOURCE... NOS $61-2-122$

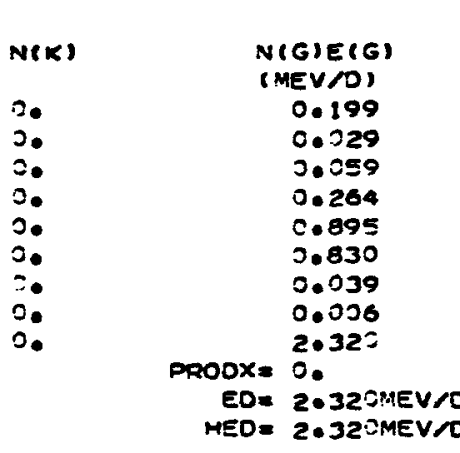

136 CS 55

$E(B) \quad N(B)$

(:MEV )

$0.341 \quad 0.926$

$0.657 \quad 2.074$

$\begin{array}{ll}0.152 & 0.190 \\ 0.270 & 0.220\end{array}$

$1.255 \quad 0.210$

$1.065 \quad 0.940$

$0.230 \quad 1.000$

TOTAL

0.200

$(K)=0.033 \mathrm{MEV}$

PHE $=3.310$

$H D H=3.310$

SOURCE... NOS $61-2-130$

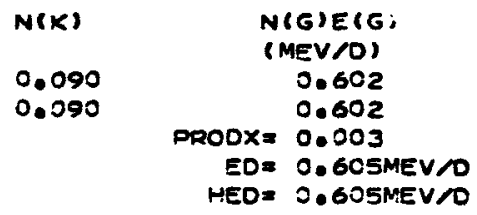

SOURCE... NOS $61-3-77$

$\begin{array}{ll}E(G) & N(G) \\ \text { (NEV) } & \\ 0.420 & 1.000 \\ 0.510 & 0.200 \\ 1.780 & 0.200 \\ \text { TOTAL } & 1.400 \\ \text { E(K) } & \\ \text { OHI } 1.0 .032 M E V & \\ \text { HPHE } 1.400 & \end{array}$

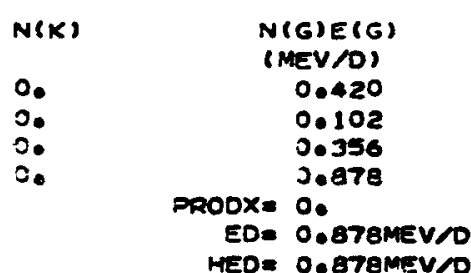

0.878MEV/O
137 SA1 56 $2.60 \mathrm{M}$

$E(\theta)$

(MEV)

$N(B)$ 
SOURCE... NOS $61-3-77$

$138 \mathrm{Cs} 55$
$32.20 \mathrm{M}$

$\begin{array}{ll}E(G) & N(G) \\ \text { (MEV) } & \\ 3.340 & 0.005 \\ 2.630 & 0.090 \\ 0.193 & 0.008 \\ 1.010 & 0.250 \\ 0.550 & 0.020 \\ 0.229 & 0.016 \\ 0.139 & 0.020 \\ 0.870 & 0.040 \\ 0.411 & 0.030 \\ 2.210 & 0.180 \\ 0.463 & 0.230 \\ 1.426 & 0.730 \\ \text { TOTAL } & 1.679 \\ \text { E(K) } & 0.033 \text { MEV } \\ \text { OH= } 1.679 & \\ \text { HPH } 1.679 & \end{array}$

$\begin{array}{lc}N(K) & N(G) E(G) \\ 0 . & (M E V / D) \\ 0 . & 0.017 \\ 0 . & 0.237 \\ 0 . & 0.002 \\ 0 . & 0.252 \\ 0 . & 0.044 \\ 2 . & 0.004 \\ 0 . & 0.003 \\ 0 . & 0.035 \\ 0 . & 0.012 \\ \partial . & 0.392 \\ 0 . & 0.105 \\ O . & 1.041 \\ 2 . & 2.150 \\ & \text { PRODX } \\ & \text { ED }=2.150 M E V / O \\ & H E D=2.150 M E V / C\end{array}$

$\begin{array}{ll}\text { E(B) } & N(B) \\ \text { (MEV) } & \\ 1.490 & 0.015 \\ 2.200 & 0.100 \\ 2.390 & 0.360 \\ 2.530 & 0.050 \\ 2.620 & 0.160 \\ 2.940 & 0.120 \\ 3.400 & 0.210\end{array}$

SOURCE... M.A. WAMLGREN ANO 'N०W. MEINKE, J. INORG. NUCL. CHEM. 24, 1527 (1962).

139 XE 54

$41.00 \mathrm{~s}$

$\begin{array}{lc}N(X) & N(G) E(G) \\ 0 . & (M E V / D) \\ 0 . & 0.034 \\ 0 . & 0.108 \\ 0 . & 0.077 \\ 0 . & 0.039 \\ & 0.258 \\ & P Q O D X=0 . \\ E D= & 0.258 M E V / O \\ H E D= & 0.258 M E V / D\end{array}$

$\varepsilon(B)$

N(B)

$\begin{array}{ll}\text { (MEV) } & \\ 0.170 & 0.200\end{array}$

0.220

0.295

0.260

TOTAL

$(K)=0.032 \mathrm{VEV} \quad 1.050$

$P 4=1.050$

$\mathrm{HPH}=1.050$

HED $=0.258 M E V / D$

SOURCE... NDS 61-3-86

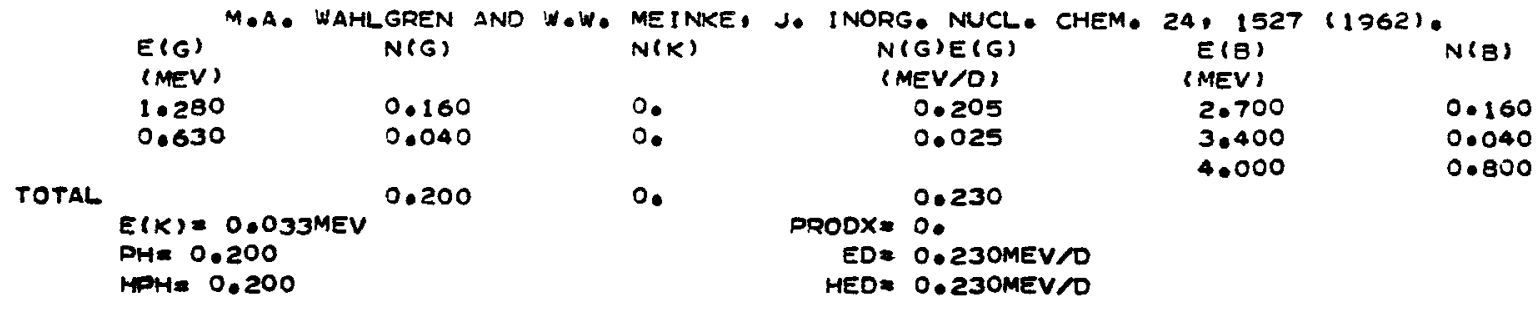


SOURCE... NOS $61-3-86$

$\begin{array}{ll}E(G) & N(G) \\ \text { (MEV) } & \\ 1.340 & 0.000 \\ 1.430 & 0.003 \\ 1.260 & 0.000 \\ 1.090 & 0.000 \\ 0.166 & 0.233 \\ \text { TOTAL } & 0.237 \\ \text { E(K) } & 0.034 \text { MEV } \\ \text { OH= } 0.294 & \\ \text { HPH }=0.294 & \end{array}$

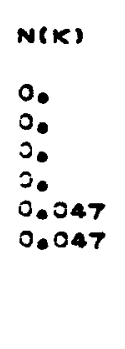

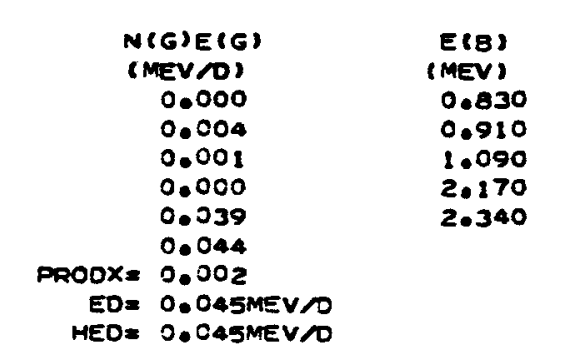

SOURCE... M.A. 'WAHLGREN AND 'Y,W. MEINKE, J. INORG. NUCL. OHEM. 24,1527 (1962).

\begin{tabular}{|c|c|c|c|}
\hline & & $\begin{array}{l}E(G) \\
(\text { MEV) } \\
0.610\end{array}$ & $\begin{array}{l}N(G) \\
1.000\end{array}$ \\
\hline & TOTAL & $\begin{array}{l}E(K)=0.033 \text { MEV } \\
P H=1.000 \\
H P H=1.000\end{array}$ & 1.000 \\
\hline
\end{tabular}

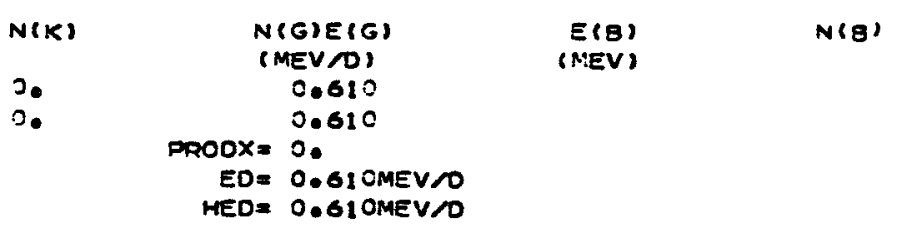

SOURCE... NOS S9-1-83

$\begin{array}{ll}E(G) & N(G) \\ (M E V) & \\ 0.537 & 0.250 \\ 0.430 & 0.045 \\ 0.306 & 0.033 \\ 0.162 & 0.073 \\ 0.030 & 1.050 \\ \text { TOTAL } & 1.451 \\ E(K)=0.034 M E V & \\ \text { PH= } 1.608 & \\ \text { HPH }=1.608 & \end{array}$

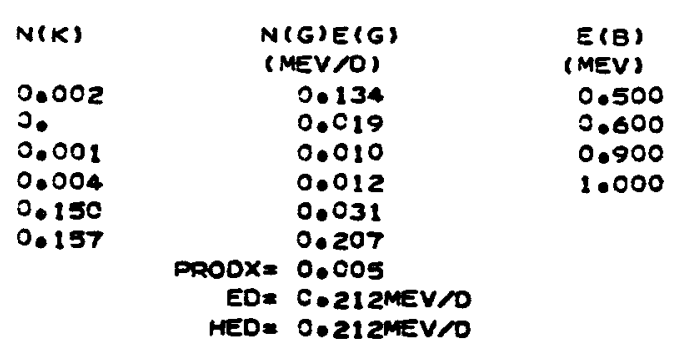

N(B)

0.000

0.003

0.000

0.280

0.720

140 CS 55

66.005
$140 \mathrm{BA} 56$

12.800
39 BA 56
$83.00^{M}$

$N(B)$

0.250

0.100

0.050

0.650 
SOURCE... NOS 59-1-83

140 LA 57

$\begin{array}{ll}E(G) & N(G) \\ \text { (MEV) } & \\ 1.597 & 1.000 \\ 0.329 & 0.224 \\ 0.431 & 0.040 \\ 0.486 & 0.432 \\ 0.752 & 0.034 \\ 0.915 & 0.280 \\ 0.868 & 0.050 \\ 0.926 & 0.110 \\ 2.343 & 0.008 \\ 2.550 & 0.050 \\ 3.000 & 0.001 \\ \text { TOTAL } & 2.229 \\ \text { E(K) } 20.035 M E V & \\ O H=2.247 & \\ H O H=2.247 & \end{array}$

$\begin{array}{lc}N(K) & N(G) E(G) \\ 0.001 & (M E V / D) \\ 0.011 & 1.597 \\ 0.0 & 0.074 \\ 0.004 & 0.017 \\ 0.0 & 0.210 \\ 0.002 & 0.026 \\ 0.0 & 0.228 \\ 0.0 & 0.043 \\ 0.0 & 0.102 \\ 0.0 & 0.019 \\ 0.0 & 0.127 \\ 0.018 & 0.003 \\ & 2.446 \\ & \text { PRODX= } 0.001 \\ & E D=2.447 M E V / O \\ & H E D=2.447 M E V / O\end{array}$

$40.20 \mathrm{H}$

$N\left(B^{3}\right.$

E(B)
(MEV)

0.420

0.830

$1 \cdot 100$

1.380

1.710

2.200

0.160

0.120

0.260

0.450

0.100

0.070

$H E D=2.447 M E V / O$
SOURCE... C.F. MILLER, USNROL-TR-160, (1957).

$141 \mathrm{BA} 56$

$18.00:$

$N(3)$

2.800

1.000

ED $=0.700$ MEV

$E(K)=0.034 \mathrm{MEV}$

PH $=1.000$

$\mathrm{HPH}=1.000$

1.000

2.

N(G)E (G)

0.700

0.700

HED $=0.700 M E V / D$

SOURCE... NOS $61-4-29$

$\begin{array}{ll}E(G) & N(G) \\ (M E V) & \\ 1.370 & 0.020 \\ \text { TOTAL } & \\ E(K)=0.035 M E V & 0.020 \\ P H=0.020 & \\ \text { HPH }=0.020 & \end{array}$

SOURCE... NOS $61-4-29$

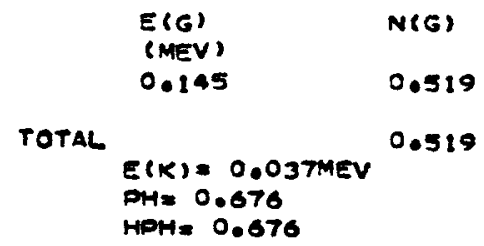

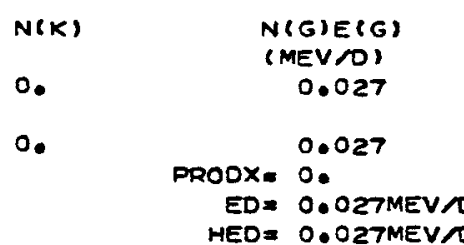

$\varepsilon(B)$

(MEV)

2.510

0.910

$E D=0.027 M E V / D$

$\begin{array}{cc}N(K) & N(G) E(G) \\ & (M E V / D) \\ 0.157 & 0.075 \\ & \\ 0.157 & 0.075 \\ & \text { PRODX }=0.006 \\ & E D=0.081 M E V / D \\ & \text { MED }=0.081 M E V / D\end{array}$

141 CE 58

$E(B) \quad N(B)$

(MEV)

0.435

0.700

\footnotetext{
33.000
}

141 LA 57 $3.80 \mathrm{H}$
0.950 0.050 
SOURCE... C.F. MILLER, USNROL-TR-160, \$19STI.

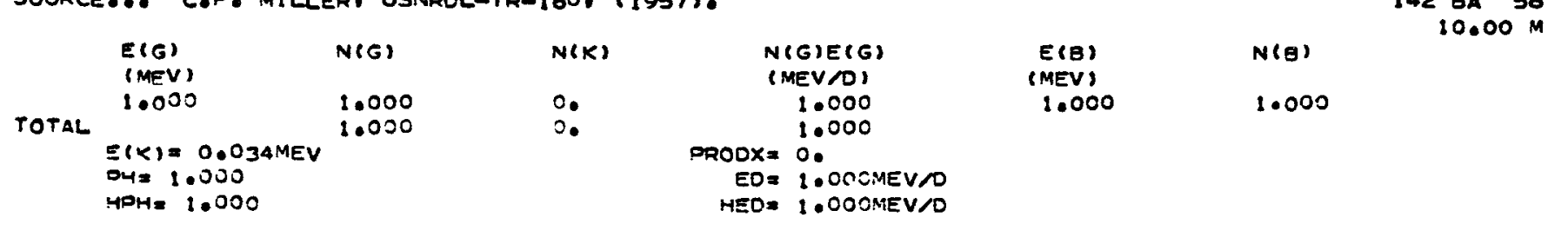

SOURCE... R.P. SCHUMAN, E.H. TURK AND R.L. HEATH, PHYS. REV, $115,185(1959)$

142 LA 57

$1.40 \mathrm{H}$

\begin{tabular}{|c|c|}
\hline $\begin{array}{l}E(G) \\
(\mu=V)\end{array}$ & $N(G)$ \\
\hline 0.540 & 0.550 \\
\hline 0.900 & 0.120 \\
\hline 1.055 & 0.050 \\
\hline 1.540 & 0.040 \\
\hline 1.750 & $0.02=$ \\
\hline 1.920 & 0.060 \\
\hline 2.000 & 0.030 \\
\hline 2.400 & 0.110 \\
\hline 2.615 & 0.030 \\
\hline 3.000 & $0.04 \mathrm{C}$ \\
\hline 3.300 & 0.007 \\
\hline 3.650 & 0.012 \\
\hline\langle\rangle$=$ & 1.084 \\
\hline
\end{tabular}

$N(K)$
0.
$0:$
0.
$0:$
0.
0.
0.
0.
0.
0.
0.
0.
0.

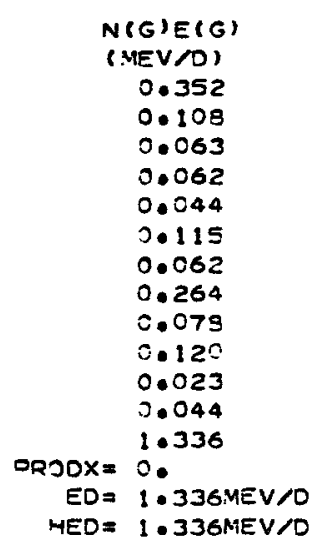

$E(B)$

$N(B)$

(MEV)

$4.400-0$.

120

.063

025

v

SOURCE... C.F. MILLER, USNROL-TR-160, (1957).

$\begin{array}{cl}E(G) & N(G) \\ \text { (MEV) } & \\ 1.200 & 1.000 \\ \text { TOTAL } & 1.000 \\ E(K)=0.035 \mathrm{MEV} & \\ P H=1.000 & \\ M P H=1.000 & \end{array}$

\begin{tabular}{|c|c|c|}
\hline$N(K)$ & N & $\begin{array}{l}Y(G) E(G) \\
(M E V / D)\end{array}$ \\
\hline 0. & & $\begin{array}{l}1.200 \\
1.200\end{array}$ \\
\hline & $\begin{array}{r}\text { PRODX }= \\
E D= \\
\text { MED }=\end{array}$ & $\begin{array}{l}=0 . \\
=1.200 M E V / O \\
=1.200 M E V / O\end{array}$ \\
\hline
\end{tabular}

$\begin{array}{ll}E(B) & N(B) \\ (M E V) & 1.000 \\ 2.300 & \\ & \end{array}$

143 LA 57

$18.00 \mathrm{n}$ 
SOURCE..O G.N. RAO ANO H.S. HANS, NUCL. PHYS, A1, 511 (1963),

143 CE 58

$33.00 \mathrm{H}$

$\begin{array}{lll}E(G) & N(G) & N(K) \\ \text { (MEV) } & & \\ 0.056 & 0.243 & 1.557 \\ 0.293 & 0.935 & 0.065 \\ 0.230 & 0.062 & 0.007 \\ 0.341 & 0.055 & 0 . \\ 0.488 & 0.058 & 0 . \\ 0.665 & 0.130 & 0 . \\ 0.718 & 0.162 & 0 . \\ 0.970 & 0.017 & 0 . \\ 1.087 & 0.012 & 0.069\end{array}$

$(K)=0.035^{4 E V}$

TOTAL

OH= 30312

$M O 4=30312$
NSGIELGR

MEV/D)

0.014

0.274

0.022

0.028

0.086

0.115

0.015

0.014

$0900 X=0.058$

$E D=0.640 M E V N O$

HED $=3.64$ CMEV

$\begin{array}{ll}\text { E(B) } & N(B) \\ \text { (MEV) } & \\ 0.300 & 0.060 \\ 0.542 & 0.050 \\ 0.730 & 0.050 \\ 1.100 & 0.400 \\ 1.400 & 0.370\end{array}$

SOURCE... J.S. GEIGER ET ALE: NUCLO PHYS, 28,387 (1961).

$\begin{array}{ll}E(G) & N(G) \\ (M E V) & \\ 0.133 & 0.100 \\ 0.053 & 0.001 \\ 0.034 & 0.012 \\ 0.100 & 0.001 \\ 0.041 & 0.013 \\ 0.090 & 0.025 \\ 0.059 & 0.012 \\ & 0.164 \\ E(K)=0.037 M E V & \\ D H=0.276 & \\ M P H=0.276 & \end{array}$

$N(K)$
0.072
0.010
0.
0.
0.0
0.030
0.00
0.112

$N(G) E(G)$
$(M E V / 0)$
0.013
$0.00 C$
0.000
0.000
0.001
0.002
0.001
0.017

PROOX $=0.004$

$E D=0.021 \mathrm{MEV} / D$

HEO $=0.021 \mathrm{MEV} / O$

SOURCE... NOS 59-1-106

$\begin{array}{ll}E(G) & N(G) \\ (M E V) & \\ 2.180 & 0.008 \\ 0.691 & 0.015 \\ 1.190 & 0.002 \\ \text { TOTAL } & 0.025 \\ \text { E(K) }=0.038 M E V & \\ \text { OHI } 0.025 & \\ \text { HPH. } 0.025 & \end{array}$

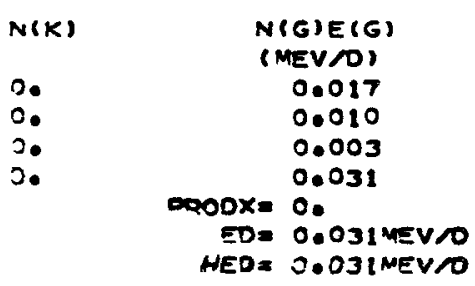


SOURCE... C.F. MILLER, USNROL-TR-160, (1957).

145 CE 58

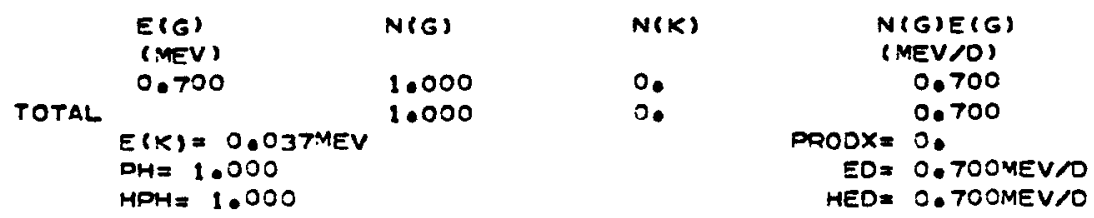

$E(B)$

(MEV)

$N(B)$

1.000

SOURCE... C.F, MILLER, USNROL-TR-160, (1957),

145 PR 59

\begin{tabular}{|c|c|c|c|c|}
\hline & $\begin{array}{l}E(G) \\
\text { (MEV) }\end{array}$ & $N(S)$ & $N(K)$ & $\begin{array}{l}N(G) E(G) \\
(Y E V D O)\end{array}$ \\
\hline & 0.200 & 1.000 & 2. & 0.800 \\
\hline IAC & $\begin{array}{l}E(K)=0.038 \mathrm{MEV} \\
P H=1.000 \\
\mathrm{HOH}=1.000\end{array}$ & 1.000 & 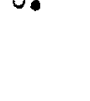 & $\begin{aligned} \text { DROOX } & =0 . \\
E D & =0.80 \text { MEV } \\
H E D & =0.80 \mathrm{JM} \text { V V }\end{aligned}$ \\
\hline
\end{tabular}

E(B)
(AEV)
I.OOO

$5.98 \mathrm{H}$

SOURCE... C.F. MILLER, USNRDL-TR-160, (1957).

\begin{tabular}{|c|c|c|}
\hline & $\begin{array}{l}E(G) \\
\text { (MEV) }\end{array}$ & $N(G)$ \\
\hline & 0.050 & 0.010 \\
\hline & $\begin{array}{l}0.110 \\
0.142\end{array}$ & $\begin{array}{l}0.090 \\
0.200\end{array}$ \\
\hline & 0.210 & 0.160 \\
\hline & 0.220 & 2.070 \\
\hline & 0.250 & 0.020 \\
\hline & 0.270 & 0.050 \\
\hline & 0.320 & 0.470 \\
\hline & & 1.070 \\
\hline & $\begin{array}{l}E(K)=0.037 \mathrm{MEV} \\
\mathrm{PH}=1.300 \\
\mathrm{HPH}=1.300\end{array}$ & \\
\hline
\end{tabular}

$\begin{array}{lc}N(K) & N(G) E(G) \\ 0.040 & (M E V / D) \\ 0.040 & 0.000 \\ 0.090 & 0.010 \\ 0.100 & 0.028 \\ 0 . & 0.034 \\ 0 . & 0.015 \\ 0 . & 0.005 \\ 0 . & 0.013 \\ 0 . & 0.15 C \\ 0.230 & 0.257 \\ & \text { DROOX }=0.008 \\ & \text { ED }=0.265 \text { MEV/D } \\ & \text { HED }=0.265 \text { MEV } 0\end{array}$

SOURCE... NOS 59-1-125

$\begin{array}{cc}E(G) & N(G) \\ (M E V) & \\ 1.490 & 0.330 \\ 0.750 & 0.460 \\ 0.460 & 1.000 \\ \text { TOTAL } & 1.790 \\ E(K)=0.038 M E V & \\ \text { PHE } 1.790 & \\ \text { MPH }=1.790 & \end{array}$

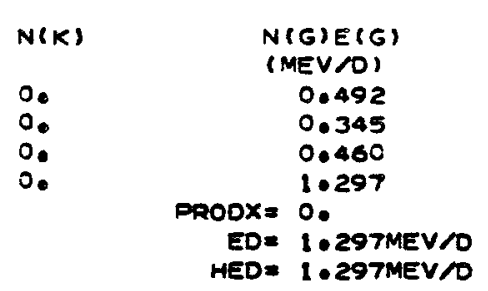

$\begin{array}{ll}E(B) & N(B) \\ \text { (MEV) } & \\ 3.700 & 0.560 \\ 2.300 & 0.440\end{array}$

146 PR 59

$24.40 \mathrm{M}$ 
SOURCE... NDS $59-1-133$

147 ND 60

11.100

\begin{tabular}{|c|c|c|}
\hline $\begin{array}{l}E(G) \\
\text { (MEV) }\end{array}$ & $N(G)$ & $N(K)$ \\
\hline $\begin{array}{l}0.532 \\
0.441 \\
0.120 \\
0.688 \\
0.600\end{array}$ & $\begin{array}{l}0.168 \\
0.019 \\
0.006 \\
0.010 \\
0.003\end{array}$ & $\begin{array}{l}0.002 \\
0.0 \\
0.005 \\
0.0 \\
0.0\end{array}$ \\
\hline $\begin{array}{l}0.277 \\
0.197 \\
0.400\end{array}$ & $\begin{array}{l}0.014 \\
2.003 \\
0.016\end{array}$ & $\begin{array}{l}0.001 \\
0.0 \\
0 .\end{array}$ \\
\hline $\begin{array}{l}0.321 \\
0.091\end{array}$ & $\begin{array}{l}3.032 \\
2.280 \\
0.551\end{array}$ & $\begin{array}{l}0.001 \\
0.504 \\
0.513\end{array}$ \\
\hline
\end{tabular}

$E(<)=0.040 \mathrm{MEV}$

$24=1.064$

1.06

$N(G) E(G)$
(MEV/DI
0.089
0.008
0.001
0.007
0.002
0.004
0.001
0.006
0.016
0.025
0.154

DRODX $=0.020$

$E D=0.174: 4 E V / O$

HED $=0.174 M E V / O$
$E(B)$

(MEV)

0.212

0.368

0.030

0.200

0.770
SOURCE... C.F. SCHWERDTFEGER, E.G. FUNK, JQ. AND J.W. NIHELICH,

148 PM 61

5.400

$(\mathrm{s})$

0.090

0.720

$\begin{array}{ll}1.900 & 0.090 \\ 1.020 & 0.720 \\ 2.460 & 0.220\end{array}$

PRODX 1.093

$E D=1.093 \mathrm{MEV} / \mathrm{O}$

HED $=1.093 \mathrm{MEV} / 0$

$\mathrm{OH}=1.032$
$\mathrm{HOH}=1.082$

0.436

.0003
0.
2.0003

(MEV/O)

0.198

0.256

SOURCE... NDS 5-2-16

$\begin{array}{ll}E(G) & N(G) \\ \text { (MEV) } & \\ 0.029 & 0 . \\ 0.096 & 0.09 \\ 0.112 & 0.024 \\ 0.1114 & 0.144 \\ 0.124 & 0.017 \\ 0.188 & 0.021 \\ 0.198 & 0.019 \\ 0.210 & 0.327 \\ 0.226 & 0.014 \\ 0.240 & 0.267 \\ 0.266 & 0.089 \\ \text { TOTAL } & 0.924 \\ \text { E(K) } & 0.040 \text { MEV } \\ \text { OHE } 1.546 & \\ \text { HOH }=1.546 & \end{array}$

$\begin{array}{lc}N(K) & N(G) E(G) \\ & (M E V / O) \\ 0.011 & 0 . \\ 0.040 & 0.003 \\ 0.086 & 0.003 \\ 0.286 & 0.016 \\ 0.043 & 0.002 \\ 0.009 & 0.004 \\ 0.005 & 0.004 \\ 0.083 & 0.069 \\ 0.006 & 0.003 \\ 0.043 & 0.064 \\ 0.011 & 0.024 \\ 0.623 & 0.189 \\ & \text { PROOX= } 0.025 \\ & E D=0.214 M E V / D \\ & \text { MED }=0.214 M E V / O\end{array}$

E(B)

N(B)

149 ND 60

$2.00 \mathrm{H}$

0.950

1.100

0.160

0.430

0.310 
SOURCE.. NOS S-2-16

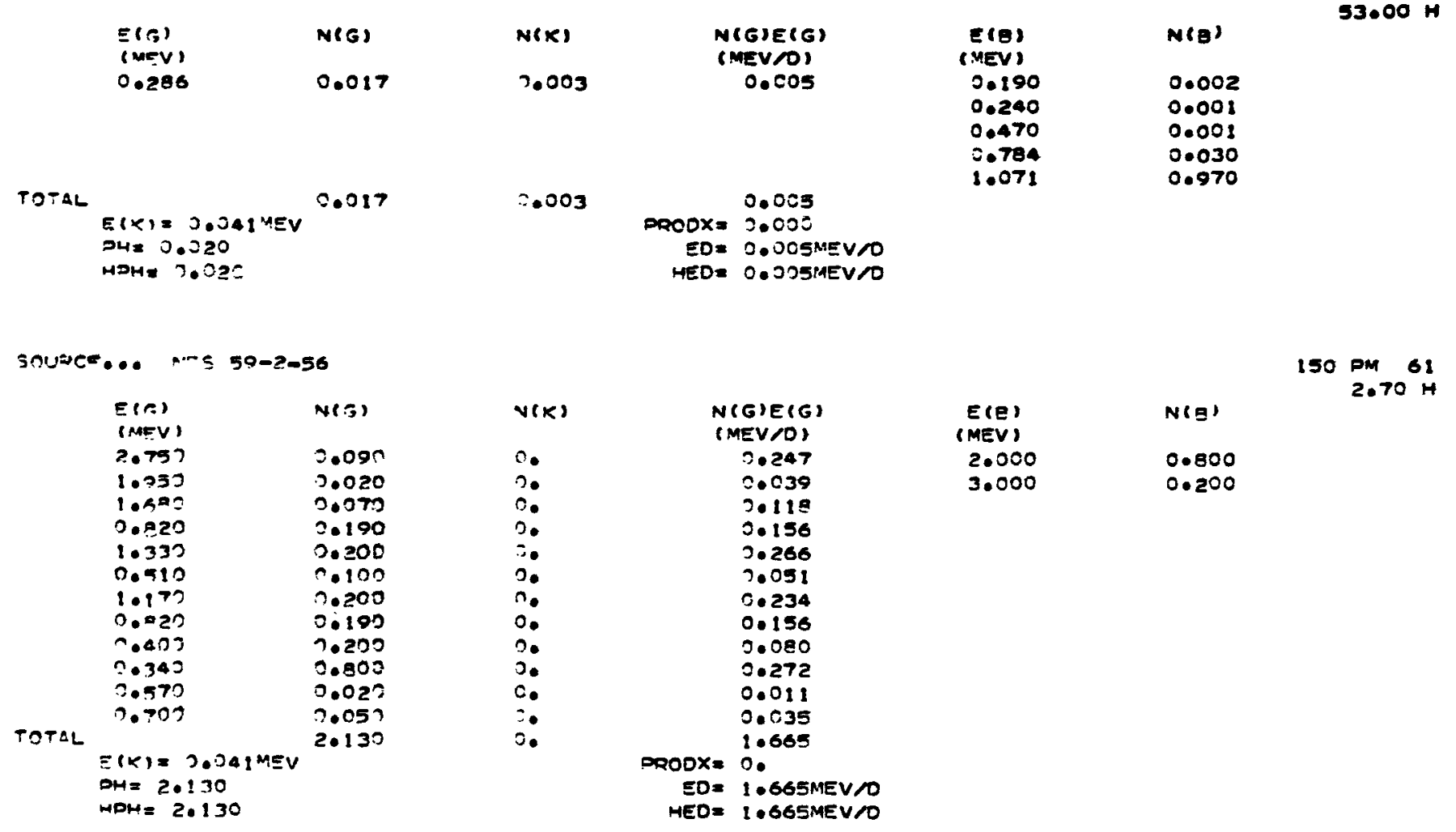

SOIJOE... C.F. MILLEQ, USMROL-TQ-180, (1957).

151 ND 60

$13.00 \mathrm{M}$

$N(K)$
0.160
0.130
0.130
0.130
0.1
0.
0.1
0.550
$404=2.250$

$v(3)$
0.090
2.120
0.120
0.120
0.250
0.500
0.500
1.700
(MEVP)

0.008

0.013

0.013

2.014

0.105

0.360

0.570

DRODX $=0.022$

ED= 1.105MEVTO

$\begin{array}{cc}\text { E(B) } & N(9) \\ \text { (MEV) } \\ 10930 & 1.000 \\ & \end{array}$


SOURCE... D.G.BURKE, M.E.LAW AND M.H.JOHMS, CAN. J. PHYS. 41.5719631.

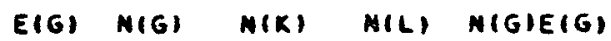

0.02570. $\begin{array}{ll}0.0351 & 0 . \\ 0.0649 & 0 .\end{array}$

0.06490.

0.06970 .

0.07620.

0.10000.

0.10480.

0.14320 .0080 .0 .00011

0.15220 .0040 .00 .0006

0.15620 .0 .00060$.

0.16320 .0240 .00510 .0010 .0039

$0.1680 \quad 0.1100 .0056 \quad 0.0010 .0185$

$\begin{array}{llllll}0.1768 & 0.052 & 0.0092 & 0.002 & 0.0092\end{array}$

$\begin{array}{lllllllll}0.2018 & 0.013 & 0 . & 0 . & 0.0026\end{array}$

$0.2090 \quad 0.0180 .00300 .0 .0038$

0.23240 .0140 .00120 .0 .0033

0.23650 .0140 .00040 .0 .0033

0.24010 .0300 .00050 .0 .0072

0.27520 .0630 .00100 .0 .0173

$0.32400 .0090 . \quad 0.0 .0029$

0.34010 .2100 .00190 .0 .0714

$0.34490 .015 \mathrm{U} 00.00052$

$0.35290 .0050 . \quad 20.0 .0013$

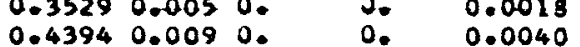

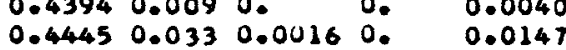

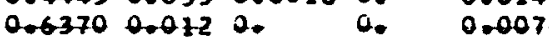

0.67000 .0120 .0 .0080

$0.71700 .0350 . \quad 0.0 .0251$

0.73500 .0080 .0 .0059

0.75200 .0010 .0 .0008

0.77200 .0010 .0 .00005

TOTAL

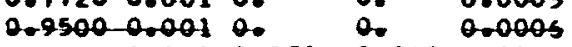

0.7010 .27110 .0990 .2193

\section{$E(K)=0.0410$ \\ $E(L)=0.0077$}

HKKLE(KL $=0.0111$

PH=1.00711

MILUE $(L)=0.0008$

$M P H=0.9721$
151 PM 61

28.4 HR

MEV

$1.20 \quad 0.11$

1.130 .13

1.630 .12

0.850 .44

0.750 .11

0.4250 .09

-


SOURCE... C.F. MILLER, USNROL-TR-160, (1957).

$152 P M 61$

$6.00 \mathrm{M}$

$\begin{array}{ll}E(E) & N(G) \\ \text { IMEV } & \\ 0.120 & 1.000 \\ 0.500 & 1.000 \\ \text { TOTAL } & 2.000 \\ E(K)=0.041 M E V & \\ \text { OHE } 2.000 & \\ \text { HOH }=2.000 & \end{array}$

$N(K)$
0.
0.
0.

SOURCE... NOS 59-4-79

$\begin{array}{ll}E(G), & N(G) \\ (M E V) & \\ 1.390 & 0.010 \\ 0.963 & 0.090 \\ 0.541 & 0.110 \\ 0.563 & 0.240 \\ 0.122 & 0.132 \\ 1.321 & 0.010 \\ 0.975 & 0.005 \\ 0.344 & 0.026 \\ \text { E(K) } & 0.000 .044 M E Y \\ \text { PH= } 0.623 & \\ \text { HPH }=0.623 & \end{array}$

SOURCE... NDS 59-4-77

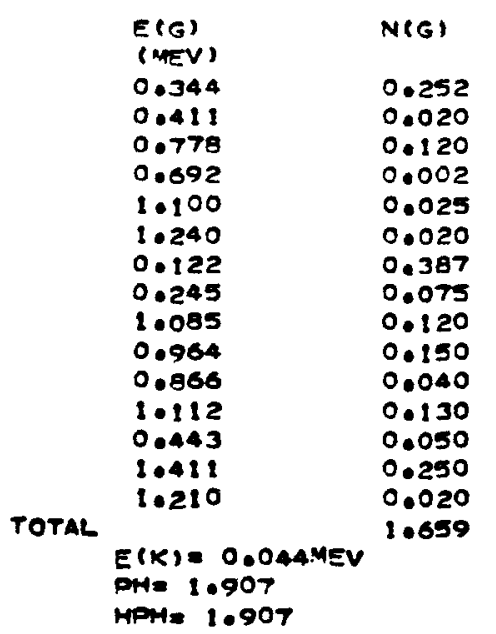

N(G)E(G)
(MEV O)
0.120
0.500
0.620
PRODX= 0.
ED= 0.620 MEV NO
HED $=0.620$ MEV O

E(B)

(MEV)

$3.200 \quad 1.000$

HED $=0.620 \mathrm{MEV} / \mathrm{O}$

152 EUI 63

$9.30 \mathrm{H}$
$N(B)$

0.000

0.016

0.000

0.020

0.740
PROOX $=0.371$

$E D=0.371 \mathrm{MEV} / \mathrm{O}$

HED $=0.371$ MEV $/ O$

(MEV)

0.560

1.260

1.550

1.870

0.016

0.013

0.005$$
0 .
$$

152 EU2 63

$13.00 \mathrm{Y}$ 
SOURCE... K. KOTAJIMA. NUCL. PHYS. 39.89 (1962).

$153 \mathrm{PM} 62$

$5.50 \mathrm{M}$

E(a)

(MEV)

$N(B)$

1.000

0.0 .094

oroox 0.139

$E D=0.139 M E V N O$

HED $=0.139 M E V / O$

DHE 1.000

HPH $=1.000$

SOURCE... NOS 59-3-55

\begin{tabular}{|c|c|c|}
\hline $\begin{array}{l}E(G) \\
(W C V)\end{array}$ & $N(G)$ & $N(K)$ \\
\hline 0.590 & 0.000 & o. \\
\hline 0.540 & 0.001 & 3.008 \\
\hline 0.191 & 0. & \\
\hline 0.107 & & \\
\hline $\begin{array}{l}0.173 \\
0.077\end{array}$ & 0.003 & $\begin{array}{l}0.013 \\
0.114\end{array}$ \\
\hline 0.054 & 0.006 & 0. \\
\hline 0.097 & 0.014 & 0. \\
\hline 0.103 & 0.143 & 0.162 \\
\hline & 2.195 & 0.297 \\
\hline
\end{tabular}

$E(K)=0.045 \mathrm{E}$

$O H=0.492$

$H P H=0.492$

SOURCE... NDS 59-3-63

E(G)

(nev)

$N(G)$

0.693

0.050

0.021

$0.725 \quad 0.206$

$0.591 \quad 0.062$

0.345

1.006

0.145

0.758

0.086

$0.998 \quad 0.114$

$\begin{array}{ll}0.475 & 0.172 \\ 0.248 & 0.062\end{array}$

TOTAL

$0.123 \quad 0.189$

$E(K)=0.044 \mathrm{MEV}$

0.189

OM= 1.549

MPHe 1.549

NRGIE(G)
(MEVTD)
0.000
0.000
0.
0.0
0.001
0.002
0.000
0.001
0.015
0.020

PRODX $=0.013$

$E D=0.032 M E V / D$

MED $=0.032 \mathrm{MEV} / O$

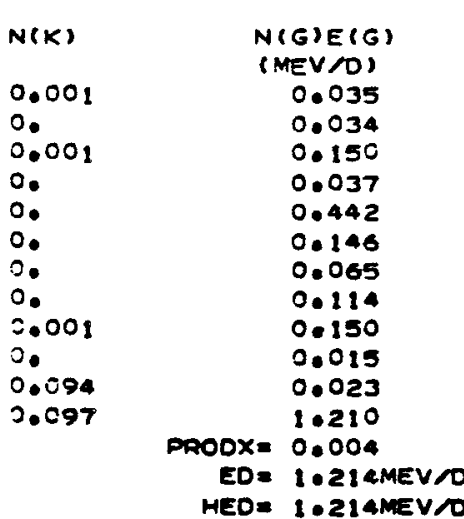

21 MMEV 0
$153 \mathrm{SM} 62$

$47.00 \mathrm{H}$

$E(B)$

(MEV)

0.130

0.640

0.698

$0 \cdot 008$

0.001

0.380

0.400
0.220

154 EU 63

$E(B)$

(MEV)

0.250

0.570

0.830

0.970

1.600

1.850

NIS

$16.00 Y$
0.290

0.350

0.200

0.030

0.030

0.100 
SOURCE... PIOS 59-3-74

$155 \mathrm{SM} 62$

$24.00 \mathrm{M}$

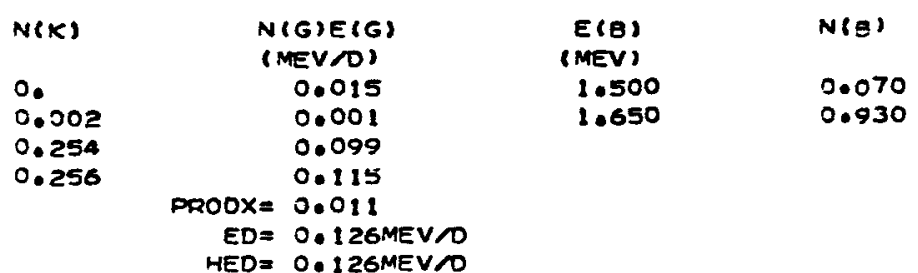

155 EU 63

$1.70 Y$

$E(s) \quad N(e)$

MEV

$0.150 \quad 0.300$

$0.160 \quad 0.400$

$0.190 \quad 0.100$

$0.250 \quad 0.200$

$\begin{array}{ll}0.132 & 0.023 \\ 0.049 & 0.018\end{array}$

TOTAL

0.181

0.042

ED $=0.050$ YEV NO

0.451

$=(K)=0.044$ KE

$\overrightarrow{T H}=0.632$

$N($ e)

156 EU 63

15.400

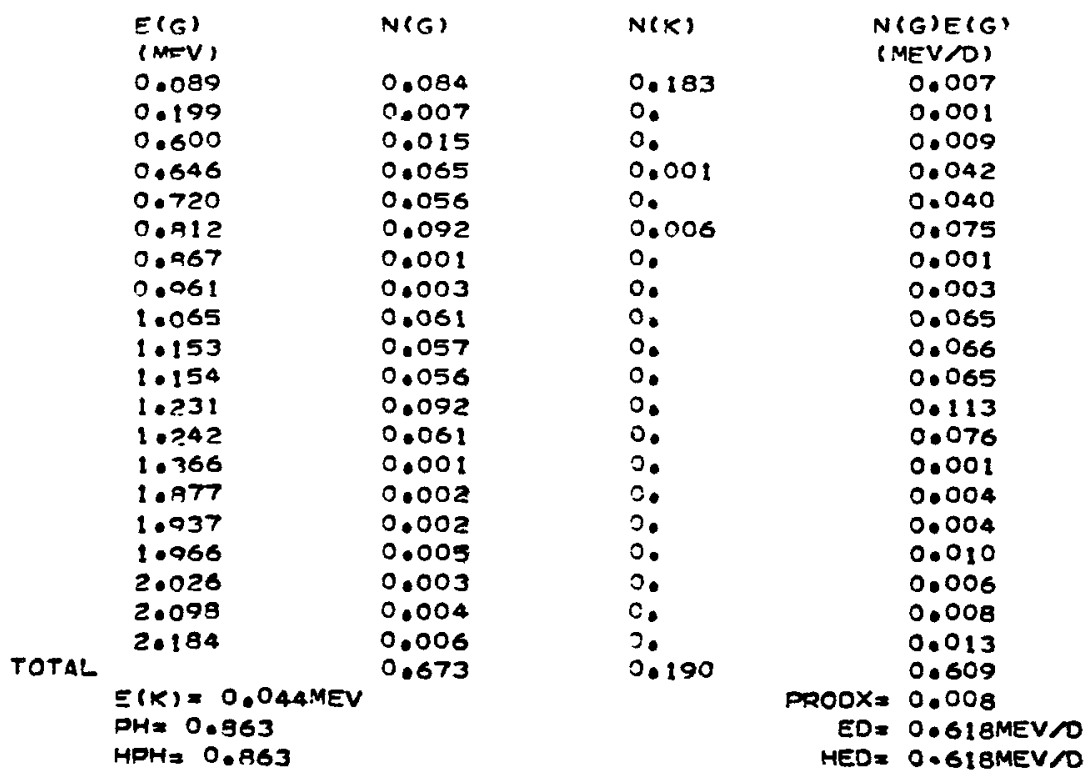

$\begin{array}{ll}\text { E(B) } & N(E) \\ \text { (MEV) } & \\ 2.430 & 0.330 \\ 1.195 & 0.140 \\ 0.485 & 0.360\end{array}$

0.360

$0.350 \quad 00.000$

HED = O.SIBMEV 
SOURCE... C.F. MILLER, USNROL-TR-160, (1957).

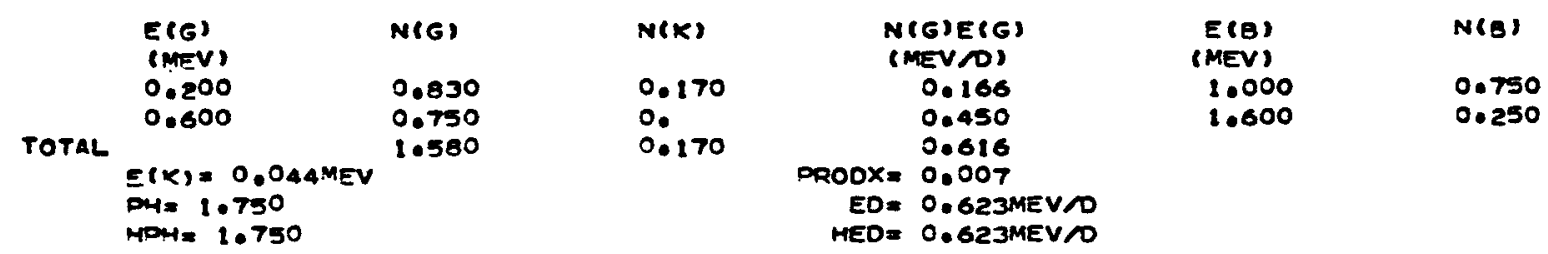

SOURCE... C.F. MILLER, USNPDL-TR-160, (1957).

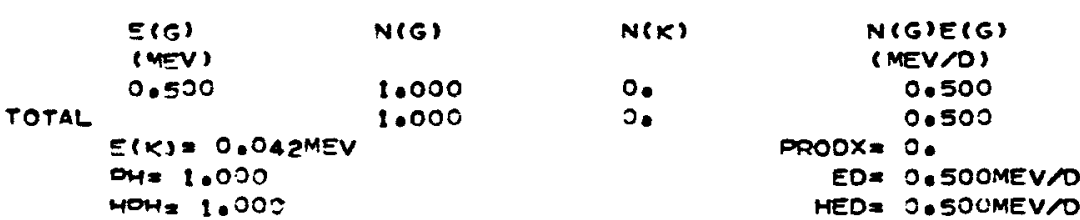

SOURCE... C.F. MILLER, USNROL-TQ-160, (1957),

158 EU 63

$$
E(B)
$$

$N(B)$

$\begin{array}{lc}N(K) & N(G) E(G) \\ \text { O. } & \text { (MEV/O) } \\ 0 . & 0.400 \\ 0 . & 0.800 \\ & 1.200 \\ & \text { OROOX }=0 . \\ & E D=1.200 M E V / O \\ \text { HEO }= & 1.200 M E V / O\end{array}$

HOH $=2.00$

SOURCE... NOS $59-5-68$

\begin{tabular}{|c|c|c|}
\hline & $\begin{array}{l}E(G) \\
(M E V)\end{array}$ & $N(G)$ \\
\hline & $\begin{array}{l}0.362 \\
0.305 \\
0.225 \\
0.137 \\
0.079 \\
0.058\end{array}$ & $\begin{array}{l}0.192 \\
0.001 \\
0.005 \\
0 . \\
0 . \\
0 .\end{array}$ \\
\hline & $\begin{array}{l}E(x)=0.045 \mathrm{MEV} \\
\text { OME } 0.201 \\
\text { HOH }=0.201\end{array}$ & 0.89 \\
\hline
\end{tabular}

$N(K)$
0.001
0.000
0.000
0.0
0.
0.000
0.002

N(G)E (G)
(MEVID)
0.070
0.000
0.001
0.
0.
0.
0.071

PRODX $=0.000$

ED $=0.071 M E V / O$

HED $=0.071 M E V / O$
1585462

$15.00 \mathrm{M}$

\section{$E(B)$}

1.000

N(S)

10000

(MEV)

1.000
1596064

$N(3)$

0.200

0.060
1890004

0.590

0.890

0.740 
SOUPCE... NDS 59-5-76

\begin{tabular}{|c|c|c|}
\hline & $\begin{array}{l}E(F) \\
(M E V)\end{array}$ & $N(G)$ \\
\hline & 0.087 & 0.072 \\
\hline & 0.197 & 0.012 \\
\hline & 0.2 .15 & 0.013 \\
\hline & 2.399 & 0.094 \\
\hline & 0.393 & 0.005 \\
\hline & O.BA2 & 0.003 \\
\hline & 0.764 & 0.007 \\
\hline & 0.079 & 0.114 \\
\hline & 0.962 & 0.036 \\
\hline & 0.966 & 0.084 \\
\hline & 0.085 & 0.003 \\
\hline & 1.070 & 3.004 \\
\hline & lonan & 0.006 \\
\hline & $1 \cdot 112$ & 0.006 \\
\hline & 1.179 & 0.054 \\
\hline & 1.200 & 0.008 \\
\hline & 1.072 & 0.029 \\
\hline & 1.714 & 0.008 \\
\hline TOTAL & - & 0.548 \\
\hline & $\begin{array}{l}E(K)=0.047 M E V \\
O H=0.568 \\
\text { HOH }=0.558\end{array}$ & \\
\hline
\end{tabular}

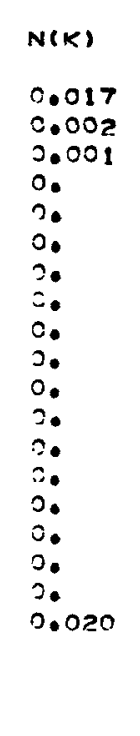

SOURCE... NDS 59-5-98

\begin{tabular}{|c|c|c|}
\hline & $\begin{array}{l}=(E) \\
(n=V)\end{array}$ & $N(G)$ \\
\hline & $\begin{array}{l}0.531 \\
0.973\end{array}$ & $\begin{array}{l}0.015 \\
0.011\end{array}$ \\
\hline & 0.106 & 0.010 \\
\hline & 0.482 & 0.019 \\
\hline & $\begin{array}{l}0.165 \\
0.361\end{array}$ & $\begin{array}{l}0.041 \\
0.590\end{array}$ \\
\hline &. .284 & 0.072 \\
\hline & 0.102 & 0.183 \\
\hline & 0.315 & 0.215 \\
\hline & 0.759 & 0.016 \\
\hline & 0.181 & 0.021 \\
\hline & $n .134$ & 0. \\
\hline & 0.078 & 0.011 \\
\hline OTAL & & 1.204 \\
\hline & $\begin{array}{l}E(K)=0.045 \mathrm{MEV} \\
O H=1.381 \\
H D H=1.381\end{array}$ & \\
\hline
\end{tabular}

160 TB 65

73.000

$N(B)$

0.120

0.190

0.380

0.110

0.200

0.004

0.005
0.100
0.035
0.081
0.003
0.004
0.006
0.007
0.064
0.010
0.037
0.011
0.403
PROOX= 0.001
$E D=0.404 \mathrm{MEV} / D$
HED $=0.404 \mathrm{MEV} / 0$

HED $=0.404 \mathrm{MEV} / D$

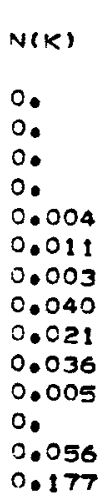

N(G)E (G)
(MEV/D)
0.008
0.003
0.001
0.009
0.007
0.213
0.021
0.019
0.068
0.004
0.004
0.01
0.001
0.357
0.008

PRODX $=0.008$

$E D=0.365 \mathrm{MEV} / \mathrm{O}$

$H E D=0.365 \mathrm{MEV} / D$
$16160 \quad 54$

$N(3)$

0.040

0.050

0.910 
SOURCE... P. GDEGOOS HANSEN, O. MIATHAN, O.E. NIELSON AND R .K. SHELINE,

E(s) NUCL. DHYS. 6. 530 (1958).

$$
\text { NiG) }
$$

N(K)

0.057

0.040

0.072

0.074

0.049

0.290

TOTAL

0.0260 .240

$E(K)=0.047 M E V$

0.642

PM= 0.736

HOM= 0.936

0.260
0.034
0.
0.
0.294

N(G)E(G)

(MEV 10 )

E(B)

E(B)

0.460

0.005

0.014

0.525

0.610

$N(3)$

161 To 55 $6.90 \mathrm{D}$

$E D=0.042 M E V / D$

HED $=0.042 M E V / D$ 
SOURCE... J.O. RASMUSSEN, F.L. CAMAVAN AMD JoMoHOLLAHDER, PHYS. REY. 107, 141 119571.

\begin{tabular}{|c|c|c|c|c|c|c|c|}
\hline $\begin{array}{l}E(G) \\
M E V\end{array}$ & $N(G)$ & $N(K)$ & NILI) & N(L2) & $N(L 3)$ & $N(M)$ & $\mid(G) E(G$ \\
\hline $\begin{array}{l}0.060 \\
0.065 \\
0.165 \\
0.208 \\
0.234 \\
0.267 \\
0.332 \\
0.335 \\
0.368 \\
0.371\end{array}$ & $\begin{array}{l}0.360 \\
0.023 \\
0.036 \\
0.240 \\
0.0- \\
0.014 \\
0.002 \\
0.00 \\
0.675\end{array}$ & 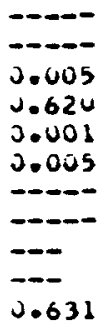 & $\begin{array}{l}0.075 \\
0.003 \\
0.002 \\
0.108 \\
-0.002 \\
-0 .\end{array}$ & $\begin{array}{l}0.149 \\
0.003 \\
0.013 \\
0.014 \\
- \\
-\end{array}$ & $\begin{array}{l}0.047 \\
0.003 \\
0.006 \\
0.001\end{array}$ & $\frac{0.085}{0.005}$ & $\begin{array}{l}0.021 \\
0.001 \\
0.006 \\
0.050\end{array}$ \\
\hline $\begin{array}{l}E(K)=0 \\
E(L 1)= \\
E(L 2)= \\
E(L 3)= \\
E(M)=0 \\
P H=1.8 \\
H P H=1 .\end{array}$ & $\begin{array}{l}.103 \\
0.022 \\
0.022 \\
0.018 \\
.006 \\
45 \\
677\end{array}$ & $\begin{array}{l}N \\
N \\
N \\
N \\
N \\
E \\
\text { He }\end{array}$ & $\begin{array}{l}K) E(K)= \\
L I \mid E(L I \\
L I E(L 2 \\
L 3) E(L 3 \\
M I E(M)= \\
=0.159 \\
D=0.158\end{array}$ & $\begin{array}{l}0.065 \\
1=0.004 \\
1=0.004 \\
1=0.001 \\
0.001 \\
\text { MEVID } \\
\text { MEVID }\end{array}$ & & & \\
\hline
\end{tabular}

2374

6.75 BA

$E(B) N(B)$

MEV

0.2480 .960

005

.084

SOURCE ... J. DORGGREEN, O.d. NIELSEN AND H. MORDBY,

238 AP 93 NUCL. PHYS. 29,515 (1962).

2.1 DA

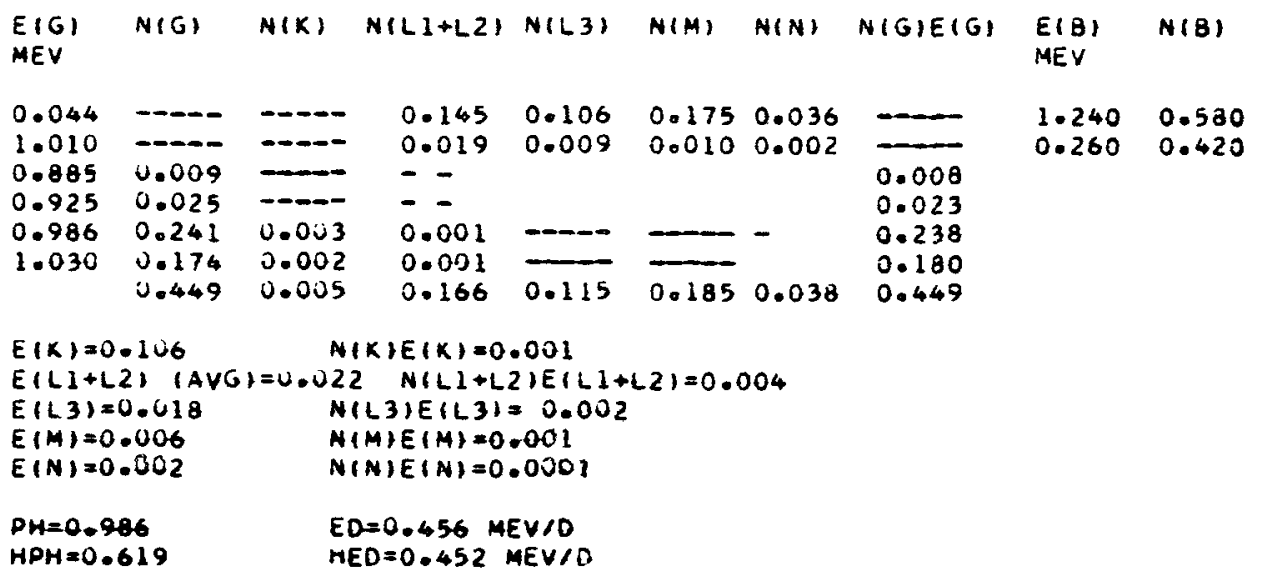


SOURCE... C.F. MILLER, USNKDL-TR-160, 119571.

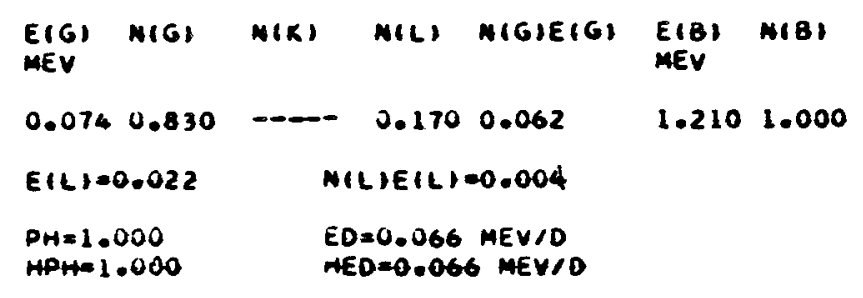

SOURCE ... C.F. MILLEH, USMRDL-TR-160, 119571 .

R.D. COMMOR AMU I LL. FAIRWEATHER.

PRO. PHYS. SOC. (LONDON) 74, 161 (1959).

$E(G)$
MEV $(G)$ M(X) N(L+M) N(G)E(G) ${ }_{\text {MEV }}$ N(B)

ळ

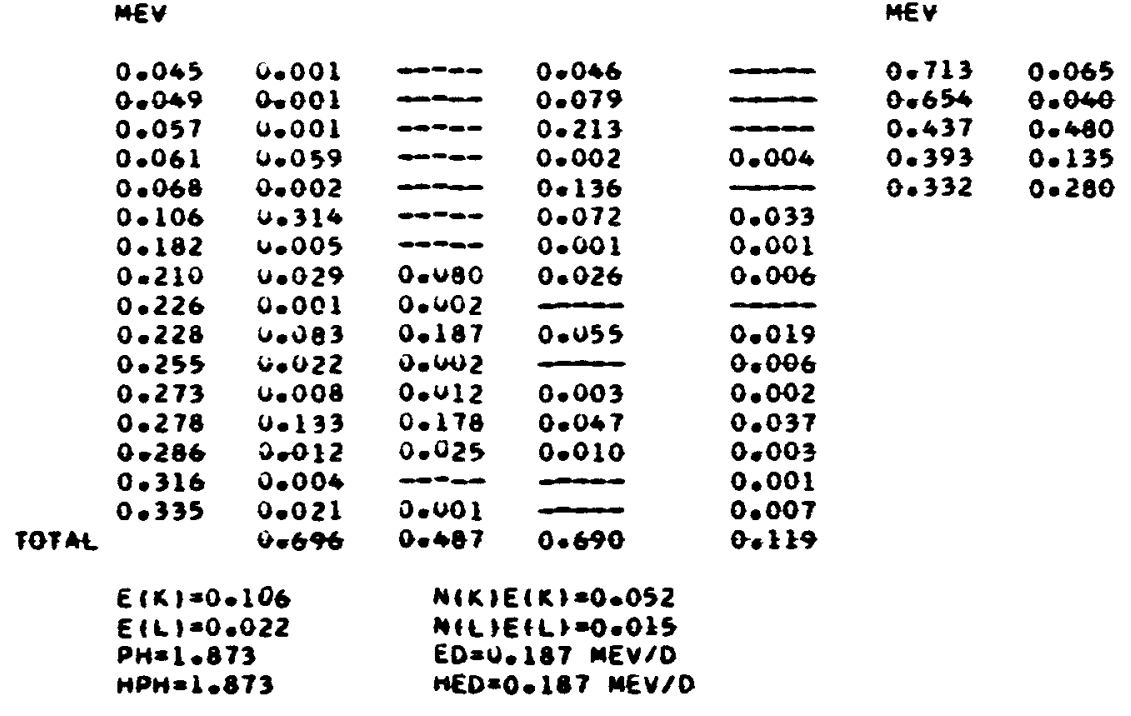

$239 \cup 92$

$23.5 \mathrm{Ml}$
239 NP 93

$56.0 \mathrm{HR}$ 
SOURCE... M.E. BUNKER, ET AL.,

PHYS. REY 116,143 (1959).

$\begin{array}{lccccc}\text { EIG) } & N(G) & N(L) & N(G) E(G) & \begin{array}{l}E(B) \\ \text { MEV }\end{array} & N(B) \\ 0.044 & 0.063 & 0.188 & 0.003 & 0.320 & 0.250 \\ & & & & & \\ 0.360 & 0.750\end{array}$

$E(L)=0.022 \quad N(L I E(L)=0.004$

$P H=0.250 \quad E D=0.007 M E V / O$

$H P H=0.250 \quad$ HED $=0.007$ MEV $/ D$
$240 \mathrm{U}$

$14.1+R$

SOURCE... M.E. BUNKER, B.J. DROPESKY, J.D. KNIGHT, J.W.STARNER 240 NP AND B.WARREN, PHYS, REV. 116,143 (1959). $7.3 \mathrm{MI}$

\begin{tabular}{|c|c|c|c|c|c|}
\hline $\begin{array}{l}E(G) \\
M E V\end{array}$ & $N(G)$ & $N(K)$ & $E(G) N(G)$ & $\begin{array}{l}E(B) \\
\text { MEV }\end{array}$ & $N(B)$ \\
\hline $\begin{array}{l}0.26 u \\
0.304 \\
0.554 \\
0.597 \\
0.758 \\
0.816 \\
0.820 \\
0.898 \\
0.936 \\
0.942 \\
1.490 \\
1.530 \\
1.620\end{array}$ & $\begin{array}{l}0.019 \\
0.009 \\
0.214 \\
0.126 \\
0.013 \\
0.016 \\
0.003 \\
0.012 \\
0.003 \\
0.0119 \\
0.015 \\
0.019 \\
0.007 \\
0.475\end{array}$ & 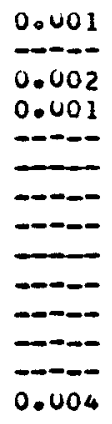 & $\begin{array}{l}0.005 \\
0.003 \\
0.119 \\
0.075 \\
0.010 \\
0.013 \\
0.002 \\
0.011 \\
0.003 \\
0.018 \\
0.022 \\
0.029 \\
0.011 \\
0.321\end{array}$ & $\begin{array}{l}2.180 \\
1.600 \\
1.300 \\
0.650\end{array}$ & $\begin{array}{l}0.520 \\
0.310 \\
0.100 \\
0.070\end{array}$ \\
\hline \multicolumn{2}{|c|}{$E(K)=0.108$} & \multicolumn{2}{|c|}{$N(K) E(K)=0.0004$} & & \\
\hline \multicolumn{2}{|c|}{$\begin{array}{l}\text { PH=0.479 } \\
H P H=0.479\end{array}$} & \multicolumn{2}{|c|}{$\begin{array}{l}E D=0.322 M E V / D \\
H E D=0.322 M E V / D\end{array}$} & & \\
\hline
\end{tabular}




\section{APPENDIX II}

\section{IEXPOSURE-DOSE RATE MULIIPLTERS}

The following list gives an exposure-dose rate multiplier for each nuclide contained in Appendix I. The point of exposure is as sumed to be 3 feet above an infinite plane uniformly contaminated with the nuclide in question. The units of the multiplier are roentgenssec-cm ${ }^{2}-\mathrm{hr}^{-1}$-disintegration-1. The multiplier will convert nuclide activities in disintegrations per second per square centimeter to exposure-dose rates in roentgens per hour. The flgure in parentheses indicates the number of zeros between the decimal point and the first significant figure; $1 . e .,(6) 840 \mathrm{is}$ to be read 0.000000840 or $0.840 \times 10^{-6}$. 


\begin{tabular}{|c|c|c|c|c|}
\hline Nuclide & Multiplier & Nucli & de & Multiplier \\
\hline $\begin{array}{l}\text { Zn } 72 \\
\text { Ga } 72 \\
\text { Ga } 73 \\
\text { Ge (1) } 73 \\
\text { Zn } 74\end{array}$ & $\begin{array}{l}(6) 840 \\
(4) 117 \\
(5) 189 \\
(7) 779 \\
(5) 365\end{array}$ & $\begin{array}{l}Y(2) \\
\operatorname{Sr} 92 \\
Y 92 \\
\operatorname{Sr} 93 \\
Y 93\end{array}$ & 91 & $\begin{array}{l}\text { (7) } 173 \\
(5) 588 \\
(5) 122 \\
(5) 114 \\
(6) 488\end{array}$ \\
\hline $\begin{array}{l}\text { Ga } 74 \\
\text { Ge (1) } 75 \\
\text { Ge (2) } 75 \\
\text { As } 76 \\
\text { Ge (1) } 77\end{array}$ & $\begin{array}{l}(4) 180 \\
(6) 303 \\
(6) 199 \\
(5) 210 \\
(6) 404\end{array}$ & $\begin{array}{l}\mathrm{Nb}(1) \\
\mathrm{Y} 94 \\
\mathrm{Zr} 95 \\
\mathrm{Nb}(1) \\
\mathrm{Nb}(2)\end{array}$ & $\begin{array}{l}93 \\
\\
95 \\
95\end{array}$ & $\begin{array}{l}\text { (6) } 166 \\
\text { (5) } 348 \\
(5) 378 \\
(5) 133 \\
\text { (5) } 391\end{array}$ \\
\hline $\begin{array}{l}\text { Ge (2) } 77 \\
\text { As } 77 \\
\text { Ge } 78 \\
\text { As (1) } 78 \\
\text { As (2) } 78\end{array}$ & $\begin{array}{l}(5) 574 \\
(7) 553 \\
(5) 243 \\
(5) 271 \\
(5) 428\end{array}$ & $\begin{array}{l}\text { No } 96 \\
\mathrm{Zr} 97 \\
\mathrm{Nb}(1) \\
\text { Nb }(2) \\
\text { Mo } 99\end{array}$ & $\begin{array}{l}97 \\
97\end{array}$ & $\begin{array}{l}(4) 115 \\
(6) 238 \\
(5) 382 \\
(5) 350 \\
(6) 658\end{array}$ \\
\hline $\begin{array}{l}\text { Se (1) } 79 \\
\text { Se (1) } 81 \\
\text { Br } 82 \\
\text { Se (1) } 83 \\
\text { Se (2) } 83\end{array}$ & $\begin{array}{l}\text { (6) } 104 \\
(6) 115 \\
(4) 143 \\
(6) 666 \\
(4) 149\end{array}$ & $\begin{array}{l}\text { Tc }(1) \\
\text { Tc } 100 \\
\text { Mo } 101 \\
\text { Tc } 101 \\
\text { Tc (1) }\end{array}$ & $\begin{array}{l}99 \\
102\end{array}$ & $\begin{array}{l}(6) 745 \\
(5) 607 \\
(5) 792 \\
(5) 193 \\
(5) 535\end{array}$ \\
\hline 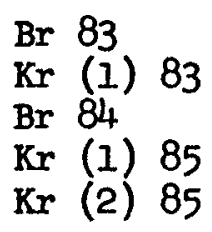 & $\begin{array}{l}\text { (6) } 277 \\
(6) 232 \\
(5) 917 \\
(6) 909 \\
(7) 277\end{array}$ & $\begin{array}{l}\mathrm{Ru} \\
\mathrm{Rh}(103 \\
\mathrm{Rh}(1) \\
\mathrm{Rh}(2) \\
\mathrm{Ru} 105\end{array}$ & $\begin{array}{l}103 \\
104 \\
104\end{array}$ & $\begin{array}{l}\text { (5) } 261 \\
(6) 122 \\
(6) 215 \\
(5) 300 \\
(5) 388\end{array}$ \\
\hline $\begin{array}{l}\mathrm{Rb}(1) \\
\mathrm{Rb}(2) \\
\mathrm{Kr} 86 \\
\mathrm{Kr} 88 \\
\mathrm{Rb} 88\end{array}$ & $\begin{array}{l}(5) 299 \\
(6) 475 \\
(5) 410 \\
(5) 768 \\
(5) 312\end{array}$ & $\begin{array}{l}\mathrm{Rh}(1) \\
\mathrm{Rh}(2) \\
\mathrm{Rh}(1) \\
\mathrm{Rh}(2) \\
\mathrm{Ru} 107\end{array}$ & $\begin{array}{l}105 \\
105 \\
106 \\
106\end{array}$ & $\begin{array}{l}(6) 417 \\
(6) 175 \\
(5) 868 \\
(5) 148 \\
(5) 122\end{array}$ \\
\hline 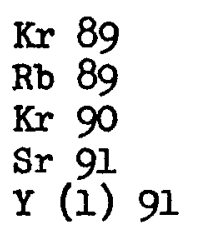 & $\begin{array}{l}(4) 169 \\
(4) 110 \\
(5) 899 \\
(5) 418 \\
(5) 282\end{array}$ & $\begin{array}{l}\mathrm{Rh}(2) \\
\mathrm{Ru} 108 \\
\mathrm{Rh} 108 \\
\mathrm{Rh}(1) \\
\mathrm{Pd}(1)\end{array}$ & $\begin{array}{l}107 \\
109 \\
109\end{array}$ & $\begin{array}{l}(5) 174 \\
(6) 265 \\
(5) 236 \\
(6) 637 \\
(6) 691\end{array}$ \\
\hline
\end{tabular}




\begin{tabular}{|c|c|c|c|c|}
\hline Nuclide & & Multiplier & Nuclide & Multiplier \\
\hline $\begin{array}{l}\mathrm{Pd}(2) \\
\mathrm{Ag}(1) \\
\mathrm{Pd}(1) \\
\mathrm{Pd}(2) \\
\mathrm{Ag}(1)\end{array}$ & $\begin{array}{l}109 \\
109 \\
111 \\
111 \\
111\end{array}$ & $\begin{array}{l}(6) 169 \\
(7) 805 \\
(6) 340 \\
6) 890 \\
6) 377\end{array}$ & $\begin{array}{l}\mathrm{Te}(1) 127 \\
\mathrm{Te}(2) 127 \\
\mathrm{Sn} 128 \\
\text { Sb (1) } 128 \\
\text { I } 128\end{array}$ & $\begin{array}{l}(6) 514 \\
(7) 234 \\
(5) 239 \\
(5) 928 \\
(6) 490\end{array}$ \\
\hline $\begin{array}{l}\text { Ag }(2) \\
\text { Pd } 112 \\
\text { Ag } 112 \\
\mathrm{Cd}(1) \\
\mathrm{Cd}(2)\end{array}$ & $\begin{array}{l}115 \\
115\end{array}$ & $\begin{array}{l}\text { (6) } 131 \\
(6) 125 \\
(5) 324 \\
(6) 177 \\
(5) 104\end{array}$ & $\begin{array}{l}\mathrm{Sb} 129 \\
\mathrm{Te}(1) 129 \\
\mathrm{Te}(2) 129 \\
\mathrm{Xe}(1) \\
\mathrm{I} 129\end{array}$ & $\begin{array}{l}\text { (5) } 540 \\
\text { (6) } 183 \\
(5) 125 \\
(6) 430 \\
(5) 784\end{array}$ \\
\hline $\begin{array}{l}\text { In (1) } \\
\mathrm{Ag} 116 \\
\mathrm{Cd}(1) \\
\text { In (1) } \\
\text { In (2) }\end{array}$ & $\begin{array}{l}115 \\
117 \\
117 \\
117\end{array}$ & $\begin{array}{l}\text { (5) } 102 \\
(5) 643 \\
\text { (5) } 708 \\
(6) 525 \\
\text { (5) } 385\end{array}$ & $\begin{array}{l}\mathrm{Sb} 131 \\
\mathrm{Te}(1) 131 \\
\mathrm{Te}(2) 131 \\
\mathrm{I} 131 \\
\mathrm{Xe}(1) 131\end{array}$ & $\begin{array}{l}\text { (5) } 318 \\
(5) 930 \\
(5) 213 \\
(5) 221 \\
(6) 198\end{array}$ \\
\hline $\begin{array}{l}\operatorname{Sn}(1) \\
\text { In }(2) \\
\text { In }(1) \\
\text { In }(2) \\
\operatorname{Sn}(1)\end{array}$ & $\begin{array}{l}117 \\
118 \\
119 \\
119 \\
119\end{array}$ & $\begin{array}{l}(5) 122 \\
5) 116 \\
(6) 269 \\
(5) 407 \\
(6) 147\end{array}$ & $\begin{array}{l}\text { Te } 132 \\
\text { I } 132 \\
\operatorname{Te}(1) 133 \\
\operatorname{Te}(2) 133 \\
\text { I } 133\end{array}$ & 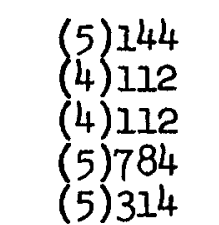 \\
\hline $\begin{array}{l}\mathrm{Cd} 120 \\
\text { In }(2) \\
\mathrm{Sb}(1) \\
\mathrm{Sb}(2) \\
\text { In (2) }\end{array}$ & $\begin{array}{l}121 \\
122 \\
122 \\
123\end{array}$ & $\begin{array}{l}\text { (5) } 271 \\
\text { (5) } 469 \\
(6) 484 \\
(5) 202 \\
(5) 535\end{array}$ & $\begin{array}{l}\mathrm{Xe}(1) 133 \\
\mathrm{Xe}(2) 133 \\
\mathrm{Te} 134 \\
\mathrm{I} 134 \\
\mathrm{Cs} \text { (1) } 134\end{array}$ & $\begin{array}{l}(6) 384 \\
(6) 306 \\
(5) 684 \\
(4) 122 \\
(6) 754\end{array}$ \\
\hline $\begin{array}{l}\mathrm{Sn}(1) \\
\mathrm{Sn}(2) \\
\mathrm{Sb}(2) \\
\mathrm{Sn}(1) \\
\mathrm{Sn}(2)\end{array}$ & $\begin{array}{l}123 \\
123 \\
124 \\
125 \\
125\end{array}$ & $\begin{array}{l}(6) 105 \\
(6) 832 \\
(5) 896 \\
(5) 196 \\
(6) 398\end{array}$ & $\begin{array}{l}\text { Cs (2) } 134 \\
I 135 \\
\mathrm{Xe}(1) 135 \\
\mathrm{Xe}(2) 135 \\
\mathrm{I} 136\end{array}$ & $\begin{array}{l}(5) 813 \\
(4) 156 \\
(5) 239 \\
(5) 149 \\
(4) 121\end{array}$ \\
\hline 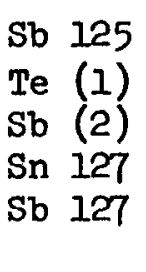 & $\begin{array}{l}125 \\
126\end{array}$ & $\begin{array}{l}\text { (5) } 254 \\
(6) 327 \\
(5) 939 \\
(5) 271 \\
(5) 263\end{array}$ & $\begin{array}{l}\mathrm{Cs} 136 \\
\mathrm{Ba}(1) 137 \\
\mathrm{Xe} 138 \\
\mathrm{Cs} 138 \\
\mathrm{Xe} 139 \\
\mathrm{Cs} 139\end{array}$ & $\begin{array}{l}(4) 117 \\
(5) 318 \\
(5) 441 \\
(5) 979 \\
(5) 146 \\
(5) 110\end{array}$ \\
\hline
\end{tabular}




\begin{tabular}{|c|c|c|c|}
\hline Nuclide & Multiplier & Nuclide & Multiplier \\
\hline Ba 139 & (6) 255 & Eu (1) 152 & (5) 192 \\
\hline Cs 140 & (5) 323 & $\mathrm{Eu}$ (2) 252 & (5) 580 \\
\hline $\mathrm{Ba} 140$ & $\left.\int 5\right) 116$ & Pm 153 & (6) 804 \\
\hline La 140 & (4) 114 & Sm 153 & (6) 186 \\
\hline $\mathrm{Ba} 141$ & (5) 365 & Eu 154 & (5) 601 \\
\hline La 141 & (6) 127 & Sm 155 & (6)726 \\
\hline Ce 141 & (6) 467 & Eu 155 & (6) 289 \\
\hline $\mathrm{Ba} 142$ & (5) 495 & Eu 156 & (5) 302 \\
\hline La 142 & (5) 601 & Eu 157 & (5) 338 \\
\hline & (5) 574 & Sm 158 & (5) 271 \\
\hline Ce 143 & (5) 350 & Eu 158 & (5)630 \\
\hline Ce 144 & (6) 123 & Gd 159 & (6) 395 \\
\hline $\operatorname{Pr} 144$ & (6) 140 & To 160 & (5) 202 \\
\hline Ce 145 & (5) 365 & Gd 161 & (5) 203 \\
\hline $\operatorname{Pr} 145$ & (5) 409 & To 161 & (6) 243 \\
\hline Ce 146 & (5) 150 & U 237 & (6) 845 \\
\hline $\operatorname{Pr} 146$ & (5) 653 & Np 238 & (5) 227 \\
\hline $\mathrm{Nd} 147$ & (6) 960 & U'239 & (6) 327 \\
\hline $\mathrm{Pm} \quad 148$ & (5) 528 & Np 239 & (6) 966 \\
\hline Nd 149 & (5) 122 & U 240 & (7) 333 \\
\hline Pm 149 & (7) 280 & Np 240 & (5) 165 \\
\hline Pm 150 & $\begin{array}{l}\text { (5) } 808 \\
(5) 560\end{array}$ & & \\
\hline $\begin{array}{ll}\text { Nd } & 151 \\
\text { Pm } & 151\end{array}$ & $\begin{array}{l}\text { (5) } 560 \\
\text { (5) } 129\end{array}$ & & \\
\hline Pm 152 & $\begin{array}{l}\text { (5) } 129 \\
\text { (5) } 340\end{array}$ & & \\
\hline
\end{tabular}




\section{APPENDIX III}

\section{SAMPLIE CALCULATIONS BASED ON THE NDS DATA FOR MASS-75 CHAIN}

Figure 1 shows the decay scheme for mass -75 nuclides as diagrammed in NDS 59-1-42. The diagram shows (1) the decay of 49-second Ge75 to 82-minute Ge75, (2) the decay of 82 -minute Ge75 to stable As75, (3) the decay of 120-day Se75 to As75 by electron capture, and (4) the decay of 1.6-hour Br75 to $\mathrm{Se} 75$ by electron capture and positron emission. The data from which the diagram was constructed are given in the data sheets which accompany it in NDS.

There 1s only one transition involved (the $0.139 \mathrm{Mev}$ transition) in the decay of 49-second Ge75 to 82-minute Ge75, and each 49-second Ge75 decay must make this transition. However, the emission of a 0.139Mev gamma photon does not accompany every decay, since the transition has a conversion coefficient of about 2 , as shown on data sheet 59-1-45. This means that two $X$ rays are emitted for every gamma photon emitted, so that the number of $0.139-\mathrm{Mer}$ gamma photons per disintegration is 0.333 and the number of $X$-ray photons is 0.667 . The $X$-ray photons have all been counted as $K X$-rays in this report, for simplicity and since the effect on dose-rate calculations is negligible, although the data sheet indicates that about one-fourth of the $X$-ray photons arise from $I$ or $M$ transitions. Also, the Auger electron emission is neglected, which would reduce the abundance of $\mathrm{KX}$-ray photons somewhat. The energy of an $X$ ray depends upon the atom emitting $1 t$, which is Ge in this case. Note that a metastable isomer may decay directly to the next element in the chain rather than by making the 1someric transition, and in this case any $X$ rays arising from conversion would be characteristic of the new element.

The gamma photons observed in the decay of 82 -minute Ge ${ }^{75}$ to $A s^{75}$, as reported by two different investigators, are listed in NDS data sheet 59-1-41 as follows: 
NRDL 182-64

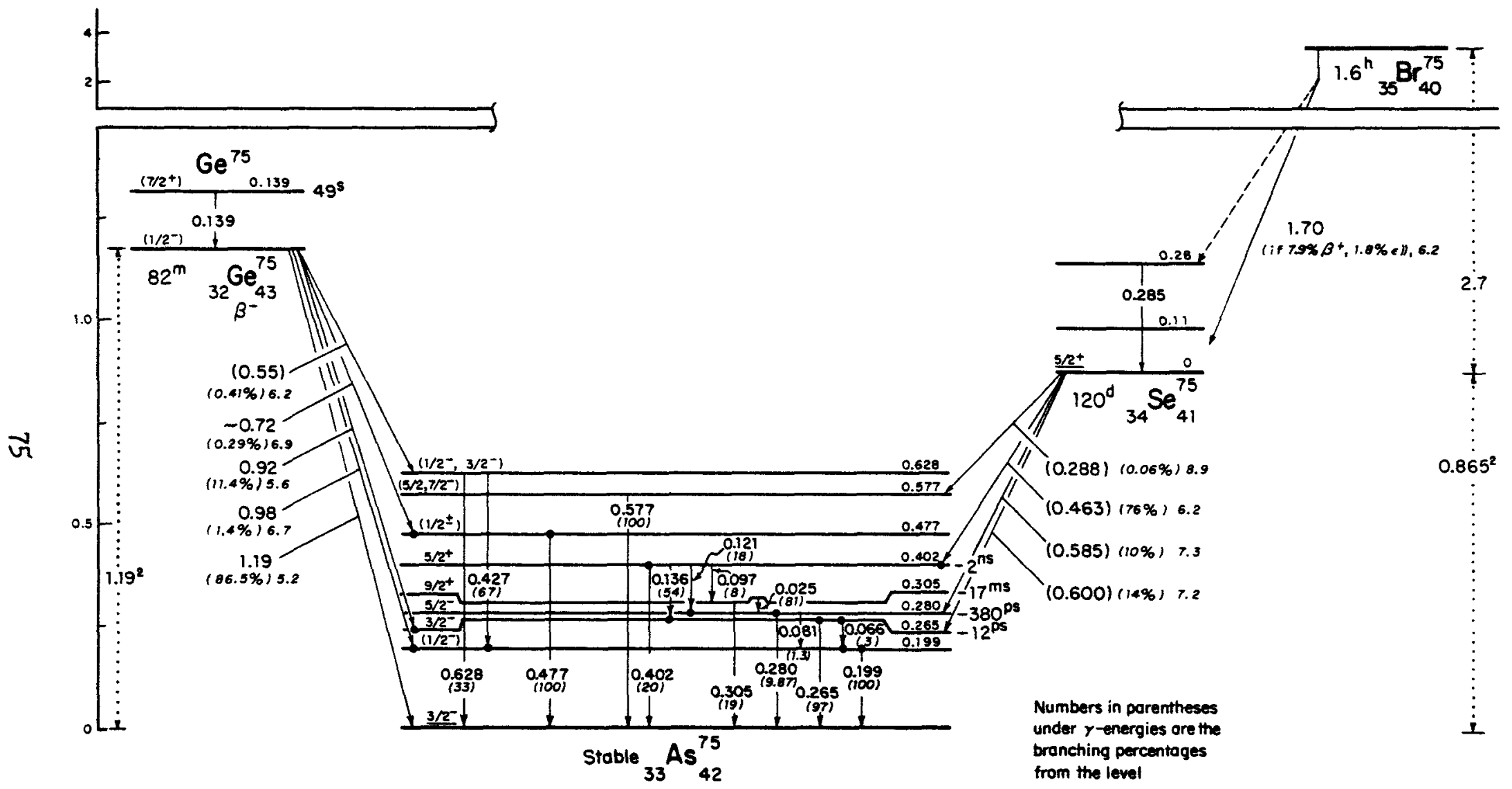

Fig. I Decay Scheme for Mass-95 Chain 


\begin{tabular}{|c|c|c|c|}
\hline \multicolumn{2}{|c|}{ Invest1gator No. I } & \multicolumn{2}{|c|}{ Investigator No. 2} \\
\hline $\begin{array}{l}\text { Energy } \\
\text { (Mev) }\end{array}$ & $\begin{array}{l}\text { Relative } \\
\text { Abundance }\end{array}$ & $\begin{array}{l}\text { Energy } \\
\text { (Mev) }\end{array}$ & $\begin{array}{l}\text { Relative } \\
\text { Abundance }\end{array}$ \\
\hline $\begin{array}{l}0.066 \\
0.199 \\
0.264 \\
0.427 \\
0.477 \\
0.628\end{array}$ & $\begin{array}{r}22 \\
120 \\
1000 \\
25 \\
23 \\
18\end{array}$ & $\begin{array}{l}0.066 \\
0.199 \\
0.265 \\
0.420 \\
0.470 \\
0.620\end{array}$ & $\begin{array}{r}25 \\
127 \\
1000 \\
26 \\
26 \\
13\end{array}$ \\
\hline
\end{tabular}

Note that the two sets of date show minor disagreements, not only in relative abundances but in the values of the energy. In such cases, IDS shows on the diagram the value which best fits the scheme, sometimes choosing an intermediate value when the reported values show wide differences.

In the case under consideration, NDS does not give absolute gammaphoton abundances. However, with the aid of the diagram, it is fairly simple to convert the relative abundances to absolute values. First, any conversion of the gammas should be noted. As it happens, the NDS data sheet for this decay carries no information on this point. A check of the data sheet for Se75 ground-state decay (NDS 59-1-49), which involves many of the same transitions, discloses appreciable conversion of the 0.066 gamma and slight conversion of the 0.199 and 0.264 gammas. The conversion coefficients, $\alpha$, are:

\begin{tabular}{ll} 
mergy & $\alpha$ \\
\cline { 2 - 2 } 0.066 & 0.4 \\
0.199 & 0.03 \\
0.264 & 0.02
\end{tabular}

Actually, NDS reports three widely separated values for the coefficient for the 0.264 gamma. We have selected the intermediate value. The conversion coefficients, $\alpha$, are simply the ratio of converted photons to unconverted photons.

From the diagram it is clear that the highest exclted level in As75 is depopulated by two gammas - the 0.628 and the 0.427 . The relative abundance ratio is about 1 to 2 and neither ray is converted. Hence, 1 out of 3 or $33 \%$ of the atoms in this level de-excite by emitting a 0.628 gamma and $67 \%$ emit the 0.427 . Since $0.41 \%$ of the ground- 
state $\mathrm{Ge}^{75}$ decays feed this level, we find $0.0041 \times 0.33=0.001$ gamma photons of $0.628 \mathrm{Mev}$ emitted per disintegration. A similar calculation yields 0.003 gammas of 0.427 Mev per disintegration.

Actually, it is generally better to normalize on the basis of a more abundant ray when possible. In the case under consideration, we note that the second level in As 75 is fed exclusively (in this decay) by the 0.92 beta ray ( $11.4 \%$ of the Ge75 decays), and the level is depopulated by the 0.265 and 0.066 gammas. The relative abundances of these two rays are about 1000 and 25, respectively, and both are converted. The relative gamma abundances do not accurately represent the relative transition probabilities, since the two transitions are converted to different extents. Each relative abundance must be multiplied by the factor $(1+\alpha)$ to convert it to a relative transition probability

$$
\begin{gathered}
25 \times(1+0.4)=35 \\
1000 \times(1+0.02)=1020
\end{gathered}
$$

Hence, about $3 \%$ of the atoms decaying to this level de-excite by the 0.066 transition while $97 \%$ make the 0.265 transition. These figures, along with the $11.4 \%$ abundance figure for feeding the level, yield the absolute numbers of each transition per disintegration. These numbers are then distributed between gamma photons and $\mathrm{X}$-ray photons according to the values of $\alpha$.

For more complicated decay schemes, the calculation may become considerably more cumbersome, but the principle remains the same; $1 . e .$, the number feeding a level must equal the number depopulating it. Needless to say, both beta branching percentages and relative abundances of the gamma rays are required for the calculation, although very weak rays can frequently be neglected. Failure to account for conversion can sometimes strongly affect the results. It should be emphasized that the whole normalization procedure depends upon the availability of a logical decay scheme for the nuclei involved. The mass-chain 75 examples just discussed are fairly typical of the kind of calculations required to obtain gamma photon abundances from the beta decay information in the Iiterature. The Nuclear Data Sheets currently being published (the compilation is continuously revised and updated) often include absolute transition probabilities marked on the decay-scheme diagram, as is the case in Figure 1 . However, many of the older sheets give only relative gamma abundances. 
GENERAL SOLUTION FOR CONVERTING REIATIVE GAMMA ABUNDANCES TO ABSOLUTE PHOTON ABUNDANCES

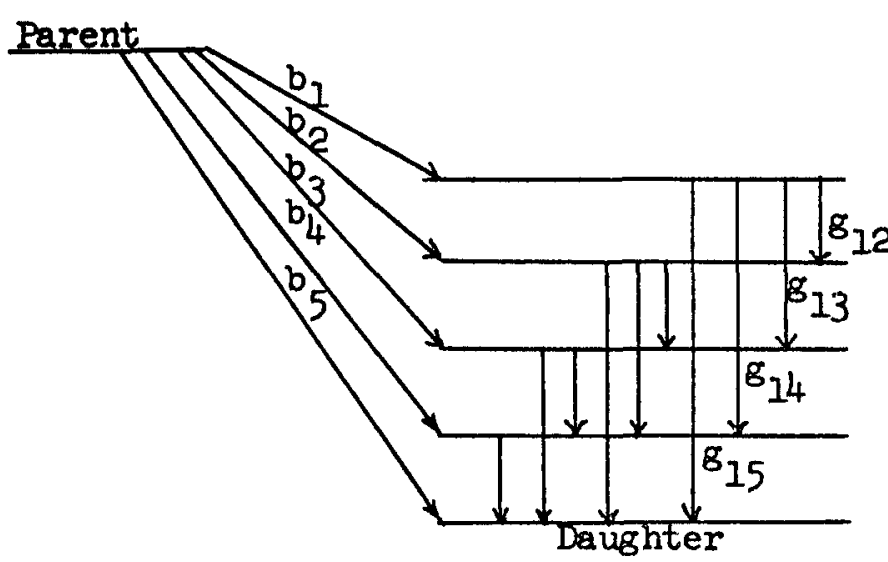

The figure at the left is a generalized decay scheme. The $b_{1}, b_{2} \ldots$ are beta branching fractions and the $g^{\prime} ' s$ are the relative abundances of the gamma photons corresponding to transitions indicated by the vertical arrows. Let $\alpha_{\mathrm{nm}}$ be the conversion coefficients for these transitions. We wish to find the absolute photon abundances (number of photons per disintegration) $\gamma_{\mathrm{nm}}$ of the ganmas.

Consider any level $i$. The population arriving at this level is:

$$
b_{i}+\sum_{k=1}^{1-1} \gamma_{k i}\left(1+\alpha_{k i}\right)
$$

where the ki include a.l gammas starting from higher levels and ending on 1. The population leaving level 1 is:

$$
\sum_{l=1+i}^{n} \gamma_{i l}\left(1+\alpha_{i \ell}\right)
$$

where $n$ Includes all levels lower than $i$. 
Since the two populations must be equal:

$$
\sum_{\ell+1}^{n} \gamma_{i \ell}\left(1+\alpha_{i \ell}\right)=b_{i}+\sum_{k=1}^{1-1} \gamma_{k 1}\left(1+\alpha_{k i}\right)
$$

Since the $g^{\prime} s$ are relative abundances, we may substitute $\gamma_{I_{I}}=\mathrm{cg}_{1}$
In this equation:

$$
\sum_{b=1+1}^{n} \operatorname{cg}_{1 \ell}\left(1+\alpha_{1 \ell}\right)=b_{1}+\sum_{k=1}^{1-1} c g_{k 1}\left(1+\alpha_{k 1}\right)
$$

But notice that for the highest-lying level, the sumation term on the right vanishes:

$$
\sum_{l=1+1}^{n} \operatorname{cg}_{1 \ell}\left(1+\alpha_{1 \ell}\right)=b_{1}
$$

and

$$
c=\frac{b_{1}}{\sum_{\ell=1+1}^{n} s_{1 \ell}\left(1+\alpha_{1 \ell}\right)}
$$

The normalizing factor, $c$, will convert any of the relative abundances to absolute photon abundances, and it can be directly determined from the beta and gamma data for the highest-lying level in the daughter nucleus. If these rays are relatively weak, however, the experimental values of the relative abundances are likely to be uncertain. In such a case, the value of $c$ determined by this method is best regarded as an estimate for calculating the gamma feed to a lower level which emits a more intense gamma ray. A better value of $c$ can then be determined from the beta and gamma data on the lower level. 
- 
INITIAL DISTRIBUTION

Copies

$\underline{\text { NAVY }}$

1

2

1

1

1

2

Chief, Bureau of Ships (Code 320-364A)

Chief, Bureau of Ships (Code 210L)

Chief, Bureau of Naval Weapons (RRRE-5)

Chief, Bureau of Yards and Docks

Chief of Naval Operations (Op-OTT)

Director, Naval Research Laboratory

Office of Naval Research, FPO, New York

CO, Naval Medical Research Institute

Supt., Naval Postgraduate School, Monterey

\section{ARMY}

Chief of Research and Development (Atomic Office)

Chief of Engineers

CG, Ballistic Research Laboratories

CO, Army Chemical Research and Development Laboratory, Md. Commandant, Chemical Center and School

Commander, Nuclear Defense Laboratory

Hq., Army Environmental Hygiene Agency

Civil Defense Unit, Army Library

Hq., Dugway Proving Ground

$\mathrm{CG}$, Quartermaster Research and Engineering Command

CO, Engineer Research and Development Laboratory (STINFO)

Director, USACDS Nuclear Group

CO, Army Office Ordnance Research (R.O. Ulsh)

$\mathrm{CG}$, Munitions Command (Picatinny Arsenal)

Office of Civil Defense, Washington

\section{AIR FORCE}

Assistant Chief of Staff Intelligence (AFNIE)

Chief, System Engineering Group(SEPIR)

Director, USAF Project RAND

Director, Air University Library, Mawwell AFB 
OTHER DOD ACTIVITIES

Director, Defense Atomic Support Agency (Library)

Commander, FC/DASA, Sandia Base (FCTG5, Library)

Director, Armed Forces Radiobiology Research Institute

Director, Weapons Systems Evaluation Group (OSD)

Defense Documentation Center

AEC ACTIVITIES AND OTHERS

Argonne National Laboratory

Atomic Bomb Casualty Commission

Atomic Energy Commission, Washington

Atomic Energy of Canada, Limited

Atomics International

Battelle Memorial Institute

Boston College (Prof. I.J. Russell)

Bureau of Mines, Washington

Committee on the Effects of Atomic Radiation

Director, Division of Biology and Medicine (AEC)

Knolls Atomic Power Laboratory

Los Alamos Scientific Laboratory (Library)

Lovelace Foundation

Massachusetts Institute of Technology

Mound Laboratory

NASA, Lewis Research Center

NASA, Scientific and Technical Information Facility

National Academy of Sciences

National Cancer Institute

Nevada Operations Office

New Brunswick Area office

New York University (Eisenbud)

Public Health Service, Washington

Sandia Corporation, Albuquerque

Sandia Corporation, Livermore

New York Operations office (AEC)

University of California Lawrence Radiation Lab., Berkeley

University of California, Los Angeles (Longwill)

University of California Lawrence Radiation Lab., Livermore

University of California (S.F. Medical Center)

University of Chicago Radiation Laboratory

University of Rochester (Atomic Energy Project)

University of Washington (Donaldson)

U.S. Weather Bureau, Las Vegas

U.S. Weather Bureau, Washington

Division of Technical Extension, Oak Ridge 
USNRDL

35

Technical Information Division

DISTRIBUTION DATE: 7 september 1965 
-

、

$\bullet$

-

. 
Naval Radiological Defense Laboratory USNRDL-TR-876

GAMMA-EMISSION DATA FOR THE CALCULATION OF EXPOSURE RATES FROM NUCLEAR DEBRIS. VOIUme Connors 10 June 196592 p. illus. 9 Connors 10 June $196592 \mathrm{p}$. 11lus. 9
refs.

Photon energies and photon abundances sion-product and other radionuclides, using data reported in the literature up to June 1963.

The data are presented in tabular form, list-

ing photon energies and abundances for gama

rays, beta rays and $X$ rays

mitted. A list

pliers is also presented for

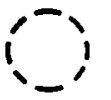

$$
\text { (over) }
$$
I. Fission Products, by G.R. Crocker and M.A. have been compiled and sumarized for some fis-
1. Fission products

2. Garma emission

3. Dose rate

4. Abundance

6. Bet a decay

7. Decay schemes

8. Radioactivity

I. Crocker, G.R.

II. Connors, M.A.

III.Title

IV.

UNCLASS IFIMD
5. Alpha decay

Naval Radiological Defense Laboratory USNRDL-TR-876

GAMA-EMISSION DATA FOR THE CALCULATION OF EXPOSURE RATES FROM NUCIEAR DEBRIS. VOlume

I. Fission Products, by G.R. Crocker and M.A. Connors 10 June 196592 p. illus. 9 refs.

UNCLASS IFIFD

Photon energies and photon abundances have been compiled and summarized for some fission-product and other radionuclides, using

dat a reported in the literature up to June 1963.

The data are presented in tabular form, list-

ing photon energies and abundances for gamma

rays, beta rays and $X$ rays

emitted. A list of multi-

pliers is also presented for

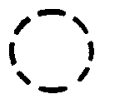

(over)

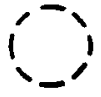

converting activities of the radionuclides to infinite-plane exposure

rates.

I,

1. Fission products

2. Gamme emission

3. Dose rate

4. Abundance

5. Alpha decay

6. Beta decay

7. Decay schemes

8. Radioactivity

I. Crocker, G.R.

II. Connors, M.A.

III.Title

IV.

UNCLASSIFTED 
Naval Radiological Defense Laboratory USNRDL-TR-876

GAMMA-EMISSION DATA FOR THE CALCULATION OF EXPOSURE RATES FROM NUCLEAR DEBRIS. VOIUmE I. Fission Products, by G.R. Crocker and M.A. Connors 10 June 196592 p. illus. 9 refs.

UNCLASSIFIED

\section{Photon energies and photon abundances} have been compiled and sumarized for some fission-product and other radionuclides, using dat a reported in the literature up to June 1963. The data are presented in tabular form, listins photon energies and abundances for rays, beta rays and $X$ rays emitted. A list of multipliers is also presented for (over)

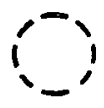

URCLASSIFIED

1. Fission products

2. Gamma emission

3. Dose rate

. Abundance

5. Alpha decay

6. Beta decay

7. Decay schemes

8. Radioactivity

I. Crocker, G.R. II. Connors, M.A. III.Title

IV.
Naval Radiological Defense Laboratory USNRDL-TR-876

GAMMA-EMISSION DATA FOR THE CALCULATION OF EXPOSURE RATES FROM NUCLEAR DEBRIS. VOIUME I. Fission Products, by G.R. Crocker and M.A. Connors 10 June 196592 p. illus. 9 refs.

Photon energies and photon abundances have been compiled and summarized for some fission-product and other radionuclides, using data reported in the literature up to June 1963. The data are presented in tabular form, listing photon energies and abundances for gammo rays, beta rays and $X$ rays

emitted. A list of multi-

pliers is also presented for (over)

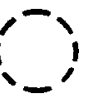

UNCIASS IFIED

Fission products

2. Gamma emission

3. Dose rate

5. Alpha decay

6. Beta decay

7. Decay schemes

8. Radioactivity

I. Crocker, G.R.

II. Connors, M.A. III. Titie

IV.

20.20

converting activities of the radionuclides to infinite-plane exposure rates.

(over)

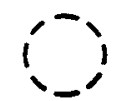

converting activities of the radionuclides to infinite-plane exposure rates. 


\section{DOCUMENT CONTROL DATA - R\&D}

(Securlty eleastfication of titlo, body of abetract and indexine ennotation must be entered when the ovorall report ie clacelfiod) 1. ORIGINATIN G ACTIVITY (Corporato author)

U. S. Naval Radiological Defense Laboratory San Francisco, California 94135

2a. REPORT SECURITY C LASBIFICATION UNCLASSIFIED

2b. GROUP

3. REPORT TITLE

GAMMA-EMISSION DATA FOR THE CALCULATION OF EXPOSURE RATES FROM NUCLEAR

DEBRIS. Volume I. Fission Products

4. DESCRIPTIVE NOTES (Typo of roport and inclunivo datoa)

5. AUTHOR(S) (Laut name, tiret name, initiat)

Crocker, Glenn $\mathrm{R}$.

Connors, M. A.

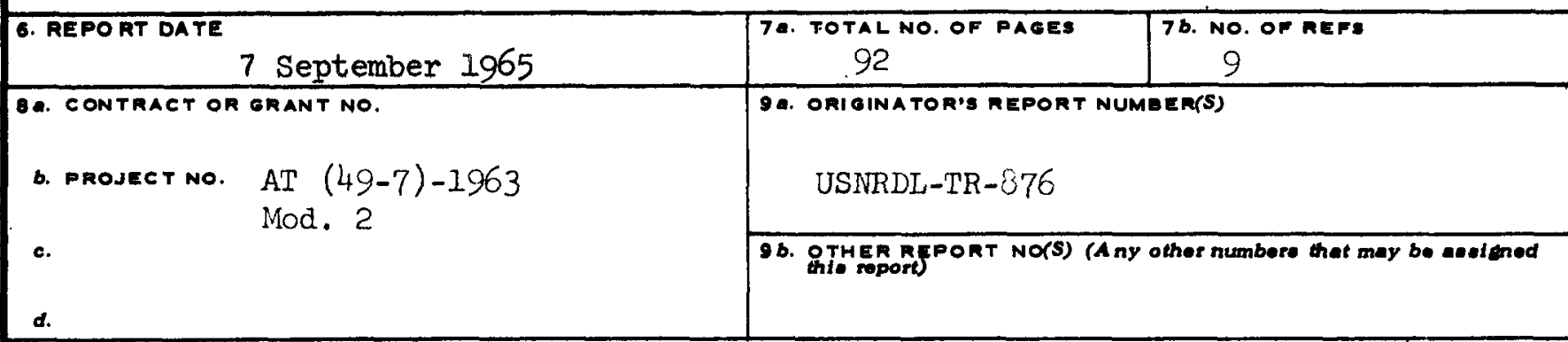

10. AVAILABILITY/LIMITATION NOTICES

Qualified requesters may obtain copies of this report from DDC.

11. SUPPL EMENTARY NOTES
12. SPONSORING MILITARY ACTIVITY

Atomic Energy Commission

Washington, D.C. 20545

13. ABSTRACT

Photon energies and photon abundances have been compiled and summarized for some fission-product and other radionuclides, using data reported in the literature up to June 1963. The data are presented in cabular form, listing photon energies and abundances for gamma rays, beta rays, and $\mathrm{X}$ rays emitted. A list of multipliers is also presented for converting activities of the radionuclides to infinite-plane exposure rates. 


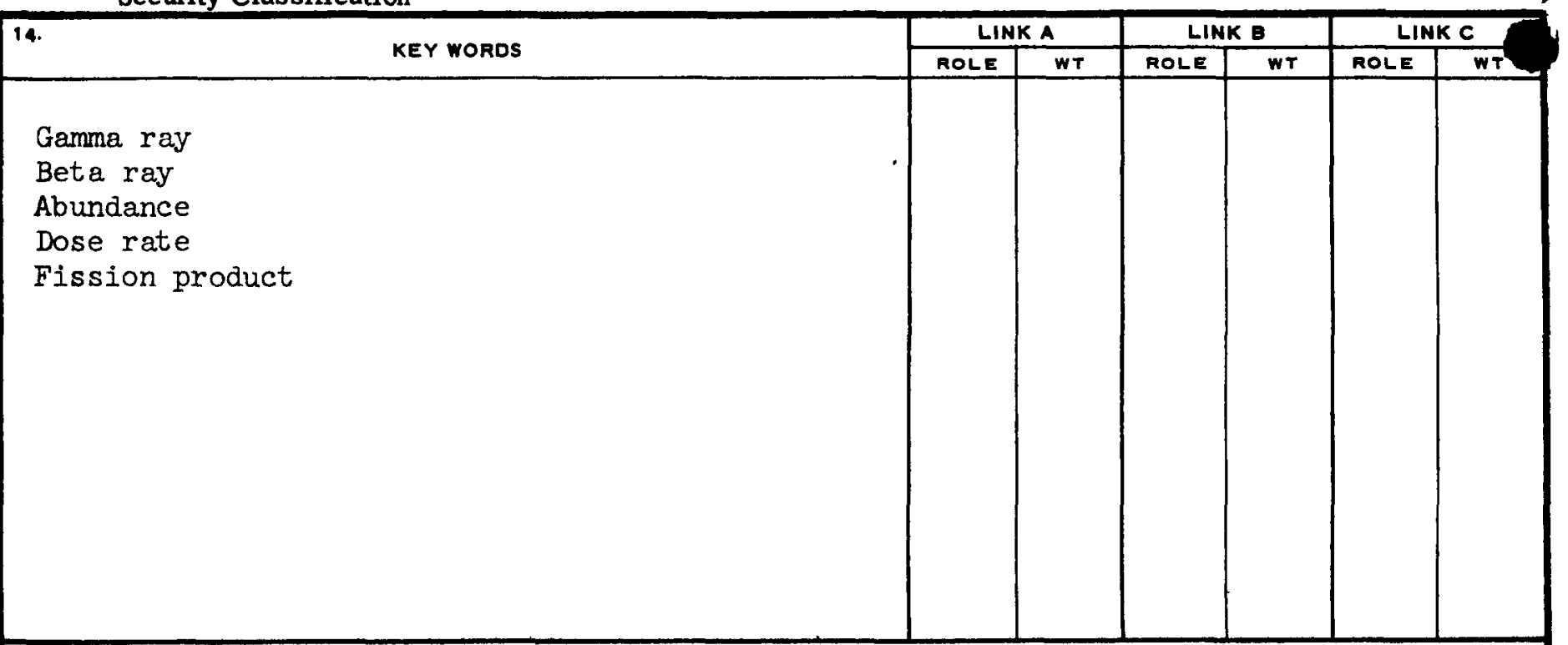

\section{INSTRUCTIONS}

1. ORIGINATING ACTIVITY: Enter the name and address of the contractor, subcontractor, grantee, Department of Defense activity or other organization (corporate author) issuing the report.

2a. REPORT SECURTY CLASSIFICATION: Enter the overall security classification of the report. Indicate whether "Restricted Data" is included. Marking is to be in accordance with appropriate security regulations.

2b. GROUP: Automatic downgrading is specified in DoD Directive 5200.10 and Armed Forces Industrial Manual. Enter the group number. Also, when applicable, show that optional markings have been used for Group 3 and Group 4 as authorized.

3. REPORT TITLE: Enter the complete report title in all capital letters. Titles in all cases should be unclassified. If a meaningful title cannot be selected without classification, show tille classification in all capitals in parenthesis immediately following the title.

4. DESCRIPTIVE NOTES: If appropriate, enter the type of report, e.g., interim, progress, summary, annual, or final. Give the inclusive dates when a specific reporting period is covered.

5. AUTHOR(S): Enter the name(s) of author(s) as shown on or in the report. Enter last name, first name, middle initial. If military, show rank and branch of service. The name of the principal disthor is an absolute minimum requirement.

6. REPORT DATE: Enter the date of the report as day, month, year; or month, year. If more than one date appears on the report, use date of publication.

7a. TOTAL NUMBER OF PAGES: The total page count should follow normal pagination procedures, i. e., enter the number of pages containing information.

7b. NUMBER OF REFERENCES Enter the total number of references cited in the report.

8a. CONTRACT OR GRANT NUMBER: If appropriate, enter the applicable number of the contract or grant under which the report was written.

8b, 8c, \& 8d. PROJECT NUMBER: Enter the appropriate military department identification, such as project number, subproject number, system numbers, task number, etc.

9a. ORIGINATOR'S REPORT NUMBER(S): Enter the official report number by which the document will be identified and controlled by the originating activity. This number must be unique to this report.

9b. OTHER REPORT NUMBER(S): If the report has been assigned any other report numbers (either by the originator or by the sponsor), al so enter this number(s).

10. AVAILABILITY/LIMITATION NOTICES: Enter any limitations on further dissemination of the report, other than those imposed by security classification, using standard statements such as:

(1) "Qualified requesters may obtain copies of this report from DDC."

(2) "Foreign announcement and dissemination of this report by DDC is not euthorized."

(3) "U. S. Government agencies may obtain copies of this report directly from DDC. Other qualified DDC users shall request through

(4) "U. S. military agencies may obtain copies of this report directly from DDC. Other qualified users shall request through

(5) "All distribution of this report is controlled Qualified DDC users shall request through ."

If the report has been furnished to the Office of Technical Services, Department of Commerce, for sale to the public, indicate this fact and enter the price, if known.

11. SUPPLEMENTARY NOTES: Use for additional explanetory notes.

12. SPONSORING MILITARY ACTIVITY: Enter the name of the departmental project office or laboratory sponsoring (paying for) the research and development. Include address.

13. ABSTRACT: Enter an abstract giving a brief and factual summary of the document indicative of the report, even though it may also appear elsewhere in the body of the technical report. If additional space is required, a continuation sheet shall be attached.

It is highly desirable that the abstract of classified reports be unclassified. Each paragraph of the abstract shall end with an indication of the military security classification of the information in the paragraph, represented as (TS), (S), (C), or (U).

There is no limitation on the length of the abstract. However, the suggested length is from 150 to 225 words.

14. KEY WORDS: Key words are technically meaningful terme or short phrases that characterize a report and may be used as index entries for cataloging the report. Key words must be selected so that no security classification is required. Identi fiers, such as equipment model desimation, trade name, mili? project code name, geographic location, may be used as key words but will be followed by an indication of technical context. The assignment of links, rales, and weights is optional. - 